\title{
LYAPUNOV SPECTRUM OF INVARIANT SUBBUNDLES OF THE HODGE BUNDLE
}

\author{
GIOVANNI FORNI, CARLOS MATHEUS, AND ANTON ZORICH
}

\begin{abstract}
We study the Lyapunov spectrum of the Kontsevich-Zorich cocycle on $\operatorname{SL}(2, \mathbb{R})$-invariant subbundles of the Hodge bundle over the support of $\mathrm{SL}(2, \mathbb{R})$-invariant probability measures on the moduli space of Abelian differentials.

In particular, we prove formulas for partial sums of Lyapunov exponents in terms of the second fundamental form (the Kodaira-Spencer map) of the Hodge bundle with respect to Gauss-Manin connection and investigate the relations between the central Oseledets subbundle and the kernel of the second fundamental form. We illustrate our conclusions in two special cases.
\end{abstract}

\section{CONTENTS}

1. Introduction

2. The Hodge bundle

2.1. The Kontsevich-Zorich cocycle

2.2. The Hodge product

2.3. Second fundamental form

2.4. Curvature

2.5. Evaluation of the second fundamental form

2.6. Variational formulas for the Hodge norm

2.7. Variational formulas for exterior powers

3. The Kontsevich-Zorich exponents

3.1. Lyapunov exponents

3.2. Formulas for the Kontsevich-Zorich exponents 26

3.3. Reducibility of the second fundamental form

4. Degenerate Kontsevich-Zorich spectrum 40

4.1. Isometric subbundles 41

4.2. A symmetry criterion 43

4.3. The central Oseledets subbundle 44

4.4. Non-vanishing of the Kontsevich-Zorich exponents

Appendix A. Lyapunov spectrum of square-tiled cyclic covers 52

A.1. Square-tiled cyclic covers 52

Date: May 26, 2018. 
A.2. Maximallly degenerate spectrum in genus four

A.3. Rank of the second fundamental form and positive exponents 54 Appendix B. Lyapunov spectrum of a higher-dimensional

$\operatorname{SL}(2, \mathbb{R})$-invariant locus $z$

B.1. Description of the locus 2

B.2. Decomposition of Hodge bundle over $Z$

B.3. Neutral Oseledets bundle versus $\operatorname{Ann}\left(B^{\mathbb{R}}\right)$ over $Z \quad 61$

Acknowledgments

References

\section{INTRODUCTION}

Consider a billiard on the plane with $\mathbb{Z}^{2}$-periodic rectangular obstacles as in Figure 1.

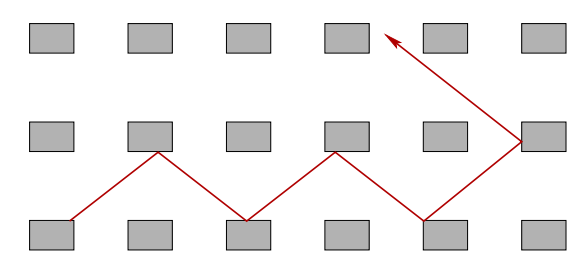

FIGURE 1. Billiard in the plane with periodic rectangular obstacles.

In [DHL], it is shown that for almost all parameters $(a, b)$ of the obstacle (i.e., lenghts $0<a, b<1$ of the sides of the rectangular obstacles), for almost all initial directions $\theta$, and for any starting point $x$ the billiard trajectory $\left\{\varphi_{t}^{\theta}(x)\right\}_{t \in \mathbb{R}}$ escapes to infinity with a rate $t^{2 / 3}$ (unless it hits the corner):

$$
\limsup _{t \rightarrow+\infty} \frac{\log \left(\text { distance between } x \text { and } \varphi_{t}^{\theta}(x)\right)}{\log t}=\frac{2}{3} .
$$

Note that changing the height and the width of the obstacle (see Figure 2) we get quite different billiards, but this does not change the diffusion rate.
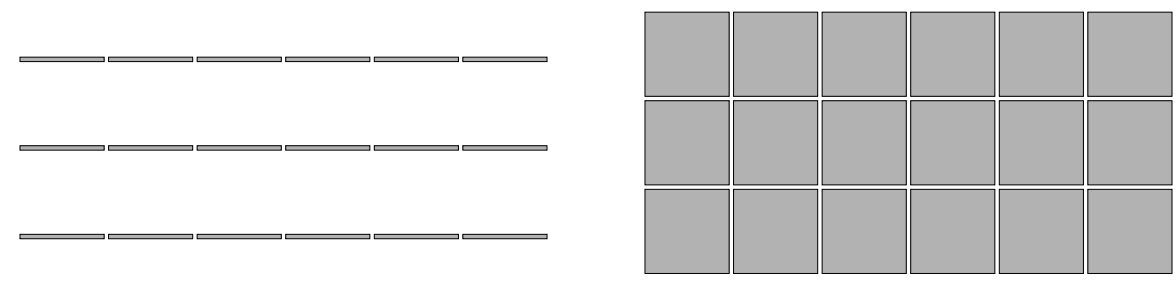

FIGURE 2. The escape rate does not depend on the size of the obstacles! 
The number " $\frac{2}{3}$ " here is the Lyapunov exponent of a certain renormalizing dynamical system associated to the initial one. More precisely, it is the Lyapunov exponent of certain subbundle of the Hodge bundle under the Kontsevich-Zorich cocycle.

The Lyapunov exponents of the Hodge bundle also govern the deviation spectrum for the asymptotic cycle of an orientable measured foliation, as well as the rate of convergence of ergodic averages for interval exchange transformations and for certain area preserving flows on surfaces see [F2], [Z0], [Z1]. The range of phenomena where the Lyapunov exponents of the Hodge bundle are extremely helpful keeps growing: nowadays, it includes, in particular, the evaluation of volumes of the moduli spaces of quadratic differentials on $\mathbb{C P}^{1}$, see [AEZ], and the classification of commensurability classes of all presently known non-arithmetic ball quotients [KM].

In this paper we develop the study of the Lyapunov spectrum of invariant subbundles of the Hodge bundle under the Kontsevich-Zorich cocycle (with respect to general $\mathrm{SL}(2, \mathbb{R})$-invariant measures). We revisit variational formulas of G. Forni from [F2] for the Hodge norm interpreting them in more geometric terms and we generalize them to invariant subbundles. We generalize Forni's formulas for partial sums of the Lyapunov exponents of the Kontsevich-Zorich cocycle in terms of geometric characteristics of the Hodge bundle (Theorem 1 in $\S[3.2$ ). We establish the reduciblity of the second fundamental form with respect to any decomposition into Hodge star-invariant, Hodge orthogonal subbundles and generalize the Kontsevich formula for the sum of all non-negative exponents (Corollary 3.5 in $\S 3.3$ ). We investigate the occurrence of zero exponents with a particular emphasis on the relation between the central Oseledets subbundle and the kernel of the second fundamental form. Our main theorem in this direction (Theorem 3 in $\$ 4.3$ ) establishes sufficient conditions for the inclusion of one into the other, hence for their equality.

We illustrate our conclusions with two examples. The first model case, inspired by recent work of A. Eskin, M. Kontsevich and A. Zorich [EKZ2], is given by arithmetic Teichmüller curves of square-tiled cyclic covers (introduced in [ [FMZ] $)$; the second model case is a certain $\operatorname{SL}(2, \mathbb{R})$-invariant locus Z (inspired by a paper of C. McMullen $\llbracket \mathbf{M c}]$ ) supporting an $\operatorname{SL}(2, \mathbb{R})$ invariant ergodic probability measure with some zero exponents.

The study of square-tiled cyclic covers was motivated by the discovery of two arithmetic Teichmüller curves of square-tiled cyclic covers with maximally degenerate Kontsevich-Zorich spectrum (see [F3], [FMt] and $[$ FMZ]). Conjecturally there are no other $\mathrm{SL}(2, \mathbb{R})$-invariant probability measures with maximally degenerate spectrum. Progress on this conjecture has been made by M. Möller [Mo] in the case of Teichmüller curves and, recently, by D. Aulicino [Au] in the general case. A conditional proof 
of the conjecture in sufficiently high genera can also be derived from a quite explicit formula for the sum of all non-negative exponents (see [EKZ1]).

The case of square-tiled cyclic covers is especially rigid. In this case, the central Oseledets subbundle is always $\operatorname{SL}(2, \mathbb{R})$-invariant, smooth and in fact coincides with the kernel of the second fundamental form. This pictures does not hold in general. In fact, in our second model case the central Oseledets subbundle does not coincide with the kernel of the second fundamental form even though it has the same rank. We emphasize that the rank of the kernel of the second fundamental form at generic points can be explained in all examples by symmetries (automorphisms) of the underlying surfaces. Indeed, the discovery of the two above-mentioned maximally degenerate examples was based on a symmetry criterion for the vanishing of the second fundamental form on the complement of the tautological subbundle (see [[F3], [FMt] and $\S 4.2$ of this paper).

This article is organized as follows. In $\S 2$, we introduce the KontsevichZorich cocycle on the Hodge bundle over the moduli space of Abelian differentials and we compute the relevant geometric tensors of the bundle, endowed with the Hodge Hermitian product, namely, the second fundamental form and the curvature of the Hermitian connection with respect to the Gauss-Manin connection. We then prove first and second variational formulas for the Hodge norm in terms of the second fundamental form and of the curvature. In $\S 3$, we derive formulas for partial sums of the Lyapunov exponents of the restriction of the Kontsevich-Zorich cocycle to any $\operatorname{SL}(2, \mathbb{R})$ invariant subbundle of the Hodge bundle. In $\S 4$ we investigate the presence of zero exponents and we prove results on the relation between the central Oseledets subbundle of the cocycle and the kernel of the second fundamental form. In Appendix A we describe the case of (arithmetic) Teichmüller curves of square-tiled cyclic covers. Finally, in Appendix B, we present our second model case. Conjecturally, this second example is representative of the general features related to the presence of zero exponents on invariant subbundles of the Hodge bundle.

\section{THE HodGE BUNDLE}

2.1. The Kontsevich-Zorich cocycle. The moduli space of Abelian differentials $\mathcal{H}_{g}$ has the structure of a complex vector bundle over the moduli space $\mathcal{M}_{g}$ of Riemann surfaces of genus $g$. The fiber over a point of $\mathcal{M}_{g}$ represented by a Riemann surface $S$ corresponds to the complex $g$-dimensional vector space of all holomorphic 1-forms $\omega$ on $S$. 
The space $\mathcal{H}_{g}$ admits a natural action of the group $\mathrm{GL}(2, \mathbb{R})$ (see, for instance, [MT] or [Z2] for an elementary description of this action). It is wellknown that the orbits of the diagonal subgroup $\left(\begin{array}{cc}e^{t} & 0 \\ 0 & e^{-t}\end{array}\right)$ of $\mathrm{GL}(2, \mathbb{R})$ project on $\mathcal{M}_{g}$ to the geodesics with respect to the Teichmüller metric. For this reason the flow on $\mathcal{H}_{g}$ defined by the action of the diagonal subgroup is called the Teichmüller geodesic flow.

The real (respectively complex) Hodge bundle $H_{\mathbb{R}}^{1}$ (respectively $H_{\mathbb{C}}^{1}$ ) over the moduli space $\mathcal{M}_{g}$ is the vector bundle having the first cohomology $H^{1}(S, \mathbb{R})$ (respectively $H^{1}(S, \mathbb{C})$ ) as its fiber over a point represented by the Riemann surface $S$. By identifying the lattices $H^{1}(S, \mathbb{Z})$ (respectively $\left.H^{1}(S, \mathbb{Z} \oplus i \mathbb{Z})\right)$ in the fibers of these vector bundles it is possible to canonically identify fibers over nearby Riemann surfaces. This identification is called the Gauss-Manin connection.

Let us consider now the pullback of the Hodge bundle to $\mathcal{H}_{g}$ with respect to the natural projection $\mathcal{H}_{g} \rightarrow \mathcal{M}_{g}$. We can lift the Teichmüller geodesic flow to the Hodge bundle by parallel transport of cohomology classes with respect to the Gauss-Manin connection, thus getting a cocycle $G_{t}^{K Z}$ called the Kontsevich-Zorich cocycle.

The Lyapunov exponents of this cocycle were proved to be responsible for some fine dynamical properties of flows on individual Riemann surfaces, see [F2], [Z1], which motivated the study of these exponents for all known Teichmüller flow-invariant (and especially $\operatorname{SL}(2, \mathbb{R})$-invariant) ergodic measures on $\mathcal{H}_{g}$.

In order to analyze the Lyapunov spectrum (i.e., collection of Lyapunov exponents) of $G_{t}^{K Z}$, we need to understand the evolution of cohomology classes $[c] \in H^{1}(S, \mathbb{R})$ under $G_{t}^{K Z}$. A particularly useful tool for this task is the Hodge norm, the main object of the next subsection.

2.2. The Hodge product. The natural pseudo-Hermitian intersection form on the complex cohomology $H^{1}(S, \mathbb{C})$ of a Riemann surface $S$ can be defined on any pair $\left(\omega_{1}, \omega_{2}\right)$ of complex-valued closed 1-forms on $S$ representing cohomology classes in $H^{1}(S, \mathbb{C})$ as

$$
\left(\omega_{1}, \omega_{2}\right):=\frac{i}{2} \int_{S} \omega_{1} \wedge \bar{\omega}_{2}
$$

Restricted to the subspace $H^{1,0}(S)$ of holomorphic 1-forms, the intersection form induces a positive-definite Hermitian form; restricted to the subspace $H^{0,1}(S)$ of anti-holomorphic 1-forms, it induces a negative-definite Hermitian form, so on the entire complex cohomology space the pseudo-Hermitian form (2.1) has signature $(g, g)$. 
For later use, we define (with the aid of the Hodge norm)

$$
\mathcal{H}_{g}^{(1)}:=\left\{\omega \in \mathcal{H}_{g}:\|\omega\|^{2}=(\omega, \omega)=1\right\},
$$

that is, $\mathcal{H}_{g}^{(1)}$ is the moduli space of unit area Abelian differentials on Riemann surfaces of genus $g$.

The Hodge representation theorem affirms that for any cohomology class $c$ in the real cohomology space $H^{1}(S, \mathbb{R})$ of a Riemann surface $S$ there exists a unique holomorphic form $h(c)$ such that $c$ is the cohomology class of the closed 1-form $\operatorname{Re} h(c)$ in $H_{\text {deRham }}^{1}(S, \mathbb{R}) \simeq H^{1}(S, \mathbb{R})$.

By the Hodge representation theorem, the positive-definite Hermitian form (2.1) on $H^{1,0}$ induces a positive-definite bilinear form on the cohomology $H^{1}(S, \mathbb{R})$ : for any $c_{1}, c_{2} \in H^{1}(S, \mathbb{R})$,

$$
\left(c_{1}, c_{2}\right):=\operatorname{Re}\left(h\left(c_{1}\right), h\left(c_{2}\right)\right) .
$$

The Hodge bundle $H_{\mathbb{R}}^{1}$ is thus endowed with an inner product, called the Hodge inner product and a norm, called the Hodge norm.

Given a cohomology class $c \in H^{1}(S, \mathbb{R})$, let $h(c)$ be the unique holomorphic 1-form such that $c=[\operatorname{Re} h(c)]$. Define $* c$ to be the real cohomology class $[\operatorname{Im} h(c)]$. The Hodge norm $\|c\|$ satisfies

$$
\|c\|^{2}=\frac{i}{2} \int_{S} h(c) \wedge \overline{h(c)}=\int_{S} \operatorname{Re} h(c) \wedge \operatorname{Im} h(c),
$$

or, in other words, $\|c\|^{2}$ is the value of $c \cdot * c$ on the fundamental cycle. The operator $c \mapsto * c$ on the real cohomology $H^{1}(S, \mathbb{R})$ of a Riemann surface $S$ is called the Hodge star operator.

We will denote the Hodge inner product of cycles $c_{1}, c_{2} \in H^{1}(S, \mathbb{R})$ by round brackets: $\left(c_{1}, c_{2}\right)=\left(c_{2}, c_{1}\right)$, and their symplectic product by angular brackets: $\left\langle c_{1}, c_{2}\right\rangle=-\left\langle c_{2}, c_{1}\right\rangle$. By definition (2.1), the spaces $H^{1,0}(S)$ and $H^{0,1}(S)$ are Hodge-orthogonal, hence the following relations hold:

$$
\begin{aligned}
*(* c) & =-c \\
\left\langle c_{1}, * c_{2}\right\rangle & =-\left\langle * c_{1}, c_{2}\right\rangle \\
\left(c_{1}, c_{2}\right) & =\left\langle c_{1}, * c_{2}\right\rangle \\
\left(h\left(c_{1}\right), h\left(c_{2}\right)\right) & =\left(c_{1}, c_{2}\right)+i\left\langle c_{1}, c_{2}\right\rangle
\end{aligned}
$$

2.3. Second fundamental form. Consider the complex Hodge bundle $H_{\mathbb{C}}^{1}$ over the moduli space $\mathcal{M}_{g}$ of complex structures having the complex cohomology space $H^{1}(S, \mathbb{C})$ as a fiber over the point of $\mathcal{M}_{g}$ represented by a Riemann surface $S$. This complex $2 g$-dimensional vector bundle is endowed with the flat Gauss-Manin connection $D_{H_{\mathbb{C}}^{1}}$ which preserves the Hermitian form 2.1) of signature $(g, g)$. 
The bundle $H_{\mathbb{C}}^{1}$ admits a decomposition into a direct sum of two orthogonal subbundles $H_{\mathbb{C}}^{1}=H^{1,0} \oplus H^{0,1}$ with respect to the Hermitian form (2.1). This decomposition is not invariant with respect to either the flat connection on $H_{\mathbb{C}}^{1}$ or with respect to the Teichmüller flow. The decomposition defines an orthogonal projection map $\pi_{1}$ of the vector bundles $\pi_{1}: H_{\mathbb{C}}^{1} \rightarrow H^{1,0}$.

The subbundle $H^{1,0}$ is a Hermitian vector bundle with respect to the Hermitian form (2.1) restricted to $H^{1,0}$. Consider the unique connection $D_{H^{1,0}}$ on $H^{1,0}$ compatible with the Hermitian metric in the fiber and with the complex structure on the base of the bundle. This (nonflat) connection coincides with the connection defined as a composition of the restriction of $D_{H_{\mathbb{C}}^{1}}$ to the subbundle $H^{1,0}$ composed with the projection $\pi_{1}$ :

$$
D_{H^{1,0}}=\left.\pi_{1} \circ D_{H_{\mathbb{C}}^{1}}\right|_{H^{1,0}},
$$

(see, for example, [GH], page 73).

The second fundamental form $A_{H^{1,0}}$ defined as

$$
A_{H^{1,0}}:=\left.D_{H_{\mathbb{C}}^{1}}\right|_{H^{1,0}}-D_{H^{1,0}}=\left.\left(I-\pi_{1}\right) \circ D_{H_{\mathbb{C}}^{1}}\right|_{H^{1,0}}
$$

is a differential form of type $(1,0)$ with values in the bundle of complexlinear maps from $H^{1,0}$ to $H^{0,1}$, hence $A_{H^{1,0}}$ can be written as a matrixvalued differential form of type $(1,0)$ (see, for example, [GH], page 78). In the literature, $A_{H^{1,0}}$ is also known as the Kodaira-Spencer map.

Note that we work with the pullbacks of the vector bundles $H_{\mathbb{C}}^{1}, H^{1,0}$ and $H^{0,1}$ to the moduli spaces $\mathcal{H}_{g}$ or $\mathcal{Q}_{g}$ of Abelian (correspondingly quadratic) differentials with respect to the natural projections $\mathcal{H}_{g} \rightarrow \mathcal{M}_{g}$ (correspondingly $\mathcal{Q}_{g} \rightarrow \mathcal{M}_{g}$ ).

We recall that there is a canonical identification between the tangent bundle of the moduli space of Riemann surfaces, which can be naturally described as the bundle $\mathcal{B}_{g}$ of equivalence classes of Beltrami differentials, and its cotangent bundle, which is naturally identified to the bundle $Q_{g}$ of holomorphic quadratic differentials. This identification follows from the existence of a canonical pairing between the bundle $Q_{g}$ of quadratic differentials and the bundle of Beltrami differentials given by integration. For any quadratic differential $q$ and any Beltrami differential $\mu$ on a Riemann surface $S$, the pairing is given by the formula:

$$
<q, \mu>:=\int_{S} q \cdot \mu .
$$

In fact, quadratic differentials are tensors of type $(2,0)$ while Beltrami differentials are tensors of type $(-1,1)$, hence the product of a Beltrami and a quadratic differential is a tensor of type $(1,1)$ which can be integrated. Beltrami differentials corresponding to trivial deformations of the complex 
structure are exactly those which are orthogonal to all quadratic differentials $[\mathrm{Na}]$, hence the pairing between Beltrami and quadratic differentials induces a non-degenerate pairing between the tangent bundle to the moduli space $\mathcal{M}_{g}$ of Riemann surfaces and the bundle of holomorphic quadratic differentials. The bundle of quadratic differentials is thus identified to the cotangent bundle of the moduli space of Riemann surfaces. There exists a natural map J : $\mathcal{Q}_{g} \rightarrow \mathcal{B}_{g}$ defined as follows,

$$
\mathcal{J}(q):=\left[\frac{|q|}{q}\right] \in \mathcal{B}_{g}, \quad \text { for all } q \in \mathcal{Q}_{g}
$$

which yields a canonical identification between the bundles of quadratic and Beltrami differentials, that is, between the cotangent and the tangent bundles to the moduli space $\mathcal{M}_{g}$ of Riemann surfaces. Taking into account this canonical identification, the differential form $A_{H^{1,0}}$ with values in the bundle of complex-linear maps from $H^{1,0}$ to $H^{0,1}$ defines a vector bundle map $H^{1,0} \rightarrow H^{0,1}$ over the moduli space of quadratic differentials. In other terms, for any $(S, q) \in \mathcal{Q}_{g}$ by evaluating the form $A_{H^{1,0}}$ at the tangent vector $v=q$ under the identification between the tangent bundle and the bundle of quadratic differentials, we get a complex-linear map

$$
A_{q}: H^{1,0}(S) \rightarrow H^{0,1}(S)
$$

For any Abelian differential $\omega \in \mathcal{H}_{g}$, let $A_{\omega}:=A_{q}$ be the complex-linear map corresponding to the quadratic differential $q=\omega^{2} \in Q_{g}$.

2.4. Curvature. The curvature tensor of the metric connections of the holomorphic Hermitian bundles $H^{1,0}, H^{1,0}$ are differential forms $\Theta_{H^{1,0}}, \Theta_{H^{0,1}}$ of type $(1,1)$ with values in the bundle of complex-linear endomorphisms of $H^{1,0}, H^{0,1}$ respectively, hence they can be written, with respect to pseudounitary frames, as skew-Hermitian matrices of differentials forms of type $(1,1)$ on the moduli space $\mathcal{M}_{g}$ (see $[\overline{\mathrm{GH}}]$, page 73 ).

Let $\left\{e_{1}, \ldots, e_{2 g}\right\} \subset H_{\mathbb{C}}^{1}$ be a pseudo-unitary frame with respect to the pseudo-Hermitian intersection form (2.1), that is, a frame which verifies the pseudo-orthonormality conditions

$$
\begin{array}{ll}
\left(e_{i}, e_{j}\right)=0, & \text { for } i \neq j \\
\left(e_{i}, e_{i}\right)=1, & \text { for } 1 \leq i \leq g \\
\left(e_{j}, e_{j}\right)=-1, & \text { for } g+1 \leq j \leq 2 g .
\end{array}
$$


Let us consider the connection matrix $\theta:=\theta_{H_{\mathbb{C}}^{1}}$ associated to the GaussManin connection $D_{H_{\mathbb{C}}^{1}}$. By the identities

$$
\begin{aligned}
0 & =d\left(e_{i}, e_{j}\right)=\left(D e_{i}, e_{j}\right)+\left(e_{i}, D e_{j}\right) \\
& =\left(\sum_{k} \theta_{i k} e_{k}, e_{j}\right)+\left(e_{i}, \sum_{k} \theta_{j k} e_{k}\right) \\
& =\theta_{i j}\left(e_{j}, e_{j}\right)+\bar{\theta}_{j i}\left(e_{i}, e_{i}\right)
\end{aligned}
$$

it follows that $\theta$ has a block structure

$$
\theta=\left(\begin{array}{cc}
\theta_{1} & B \\
A & \theta_{2}
\end{array}\right)
$$

with $g \times g$ blocks $\theta_{1}, \theta_{2}, A$ and $B$ that verify the relations

$$
\theta_{1}=-{\overline{\theta_{1}}}^{t}, \quad \theta_{2}=-{\overline{\theta_{2}}}^{t}, \quad B=\bar{A}^{t} .
$$

Observe that for any unitary frame $\left\{\omega_{1}, \ldots, \omega_{g}\right\} \subset H^{1,0}$, there is an associated pseudo-unitary frame $\left\{e_{1}, \ldots, e_{2 g}\right\} \subset H_{\mathbb{C}}^{1}$, with respect to the intersection form (2.1), defined as follows,

$$
\begin{array}{ll}
e_{i}=\omega_{i}, & \text { for } 1 \leq i \leq g, \\
e_{j}=\bar{\omega}_{j-g}, & \text { for } g+1 \leq j \leq 2 g,
\end{array}
$$

and, with respect to the above pseudo-unitary frame, the blocks $\theta_{1}, \theta_{2}$ are equal to the connection matrices $\theta_{H^{1,0}}, \theta_{H^{0,1}}$ of the connections $D_{H^{1,0}}$ and $D_{H^{0,1}}$, respectively, that is, $\theta_{1}=\theta_{H^{1,0}}$ and $\theta_{2}=\theta_{H^{0,1}}$, and $A=A_{H^{1,0}}$ is the matrix of the second fundamental form (see $[\mathrm{GH}]$, page 76).

Let us now consider the curvatures $\Theta_{H_{\mathbb{C}}^{1}}, \Theta_{H^{1,0}}$ and $\Theta_{H^{0,1}}$ of the connections $D_{H_{\mathbb{C}}^{1}}, D_{H^{1,0}}$ and $D_{H^{0,1}}$ on the vector bundles $H_{\mathbb{C}}^{1}, H^{1,0}$ and $H^{0,1}$ respectively. It follows from the above relations that

$$
\theta_{H_{\mathbb{C}}^{1}} \wedge \theta_{H_{\mathbb{C}}^{1}}=\left(\begin{array}{cc}
\theta_{H^{1,0}} \wedge \theta_{H^{1,0}}+\bar{A}^{t} \wedge A & * \\
* & \theta_{H^{0,1}} \wedge \theta_{H^{0,1}}+A \wedge \bar{A}^{t}
\end{array}\right) .
$$

By Cartan's structure equation (see for instance [GH], page 75) we have the identity $\Theta_{H_{\mathbb{C}}^{1}}=d \theta_{H_{\mathbb{C}}^{1}}-\theta_{H_{\mathbb{C}}^{1}} \wedge \theta_{H_{\mathbb{C}}^{1}}$, hence we conclude that

$$
\Theta_{H_{\mathbb{C}}^{1}}=\left(\begin{array}{cc}
\Theta_{H^{1,0}}-\bar{A}_{H^{1,0}}^{t} \wedge A_{H^{1,0}} & * \\
* & \Theta_{H^{0,1}}-A_{H^{1,0}} \wedge \bar{A}_{H^{1,0}}^{t}
\end{array}\right)
$$

It follows that (compare with $[\mathrm{GH}]$, page 78)

$$
\Theta_{H^{1,0}}=\left.\Theta_{H_{\mathbb{C}}^{1}}\right|_{H^{1,0}}+A_{H^{1,0}}^{*} \wedge A_{H^{1,0}} .
$$

Note that $\Theta_{H_{\mathbb{C}}^{1}}$ is the curvature of the Gauss-Manin connection, which is flat. So $\Theta_{H_{\mathbb{C}}^{1}}$ is null, and the curvature $\Theta_{H^{1,0}}$ can be written as

$$
\Theta_{H^{1,0}}=A_{H^{1,0}}^{*} \wedge A_{H^{1,0}} .
$$


Similarly to the case of the second fundamental form, we pull back the bundle $H^{1,0}$ to a point $(S, q)$ of $Q_{g}$ and take the value of the curvature form on the tangent vectors $v, \bar{v}$, where the tangent vector $v=q$ under the identification discussed above between the tangent bundle of the moduli space and the bundle of quadratic differentials. Thus, instead of a differential form of type $(1,1)$ with values in the bundle of complex endomorphisms of the bundle $H^{1,0}$, we get a section of that bundle over $Q_{g}$, that is, we get a complex endomorphism $\Theta_{q}$ of the space $H^{1,0}(S)$ for any pair $(S, q) \in \mathcal{Q}_{g}$. For any Abelian differential $\omega \in \mathcal{H}_{g}$, let $\Theta_{\omega}:=\Theta_{q}$ denote the complex endomorphism corresponding to the quadratic differential $q=\omega^{2} \in Q_{g}$. For any Riemann surface $S$ and any orthonormal basis $\Omega:=\left\{\omega_{1}, \ldots, \omega_{g}\right\}$ of the space $H^{1,0}(S)$, the system $\bar{\Omega}=\left\{\bar{\omega}_{1}, \ldots, \bar{\omega}_{g}\right\}$ is a pseudo-orthonormal basis of the space $H^{0,1}(S)$. Let $\Theta$ be the matrix of the complex endomorphism $\Theta_{\omega}$ with respect to the basis $\Omega$ and $A$ be the matrix of the second fundamental form operator $A_{\omega}$ with respect to the bases $\Omega$ and $\bar{\Omega}$. Formula (2.9) can be written in matrix form as follows:

$$
\Theta=-A^{*} \cdot A \text {. }
$$

It is immediate from the above formulas that the matrix $\Theta$ of the curvature of the Hodge bundle is a negative-semidefinite Hermitian matrix.

2.5. Evaluation of the second fundamental form. A formula for the second fundamental form $A_{H^{1,0}}$ was implicitly computed in [F2], §2. We state such a formula below. We remark that all formulas in [F2] are written with different notational conventions, which we now explain for the convenience of the reader. Any Abelian differential $\omega$ on a Riemann surface $S$ induces an isomorphism between the space $H^{1,0}(S)$ of all Abelian differentials on $S$, endowed with the Hodge norm, and the subspace of all square integrable meromorphic functions (with respect to the area form of the Abelian dif-

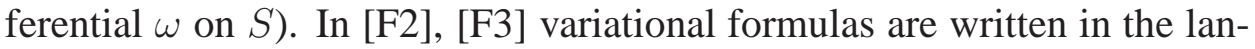
guage of meromorphic functions. In this paper we will adopt the language of Abelian differentials.

Let $(S, \omega)$ be a pair (Riemann surface $S$, holomorphic 1-form $\omega$ on $S$ ). Following [F2], §2, for any $\alpha, \beta \in H^{1,0}(S)$ we define:

$$
B_{\omega}(\alpha, \beta):=\frac{i}{2} \int_{S} \frac{\alpha \beta}{\omega} \bar{\omega} .
$$

The complex bilinear form $B_{\omega}$ depends continuously, actually (real) analytically, on the Abelian differential $\omega \in \mathcal{H}_{g}$.

Lemma 2.1. For any $\omega \in \mathcal{H}_{g}$, the second fundamental form $A_{\omega}$ can be written in terms of the complex bilinear form $B_{\omega}$, namely

$$
\left(A_{\omega}(\alpha), \bar{\beta}\right)=-B_{\omega}(\alpha, \beta), \quad \text { for all } \alpha, \beta \in H^{1,0}(S) .
$$


In particular, for any orthonormal basis $\left\{\omega_{1}, \ldots, \omega_{g}\right\}$ of holomorphic forms in $H^{1,0}(S)$ and any $\alpha \in H^{1,0}(S)$,

$$
A_{\omega}(\alpha)=\sum_{j=1}^{g} B_{\omega}\left(\alpha, \omega_{j}\right) \bar{\omega}_{j}
$$

Proof. The argument is a simplified version of the proof of Lemma 2.1 in [F2] rewritten in the language of holomorphic differentials. As everywhere in this paper we use the same notation for holomorphic (antiholomorphic) forms and their cohomology classes, while for other closed 1-forms we use square brackets to denote the cohomology class. Let $\left\{\left(S_{t}, \omega_{t}\right)\right\}$ denote a Teichmüller deformation, that is, a trajectory of the Teichmüller flow. Let $\alpha$ be any given holomorphic differential on the Riemann surface $S_{0}$. There exists $\varepsilon>0$ such that there is a natural identification $H^{1}\left(S_{t}, \mathbb{C}\right) \simeq H^{1}\left(S_{0}, \mathbb{C}\right)$ by parallel transport for all $|t|<\varepsilon$, so that locally constant sections are parallel for the Gauss-Manin connection.

Let $\left\{\alpha_{t}\right\}$ be a smooth one-parameter family of closed 1-forms such that $\alpha(0)=\alpha$ and $\alpha_{t}$ is holomorphic on $S_{t}$ for all $|t|<\varepsilon$, that is, $\left\{\alpha_{t}\right\}$ is a smooth local section of the bundle $H^{1,0}$. Let $\pi_{1}: H_{\mathbb{C}}^{1} \rightarrow H^{1,0}$ denote the natural projection (see formula (2.7)). By definition,

$$
A_{\omega}(\alpha)=\left.\left(I-\pi_{1}\right) \circ D_{H_{\mathbb{C}}^{1}}\right|_{H^{1,0}}(\alpha)=\left(I-\pi_{1}\right)\left(\left[\frac{d \alpha}{d t}(0)\right]\right) .
$$

Thus, for any 1 -form $\beta \in H^{1,0}$, in order to compute the pseudo-Hermitian intersection $\left(A_{\omega}(\alpha), \bar{\beta}\right)$, it is sufficient to compute the derivative $d \alpha / d t(0)$ up to exact 1-forms and up to 1-forms of type $(1,0)$. In fact, it follows from formula (2.12) that the cohomology class $A_{\omega}(\alpha)-[d \alpha / d t(0)] \in H^{1}(S, \mathbb{C})$ is holomophic. Hence, by the definition of the pseudo-Hermitian intersection form, it is orthogonal to $\bar{\beta}$ for any holomorphic form $\beta$,

$$
\left(A_{\omega}(\alpha)-\left[\frac{d \alpha}{d t}(0)\right], \bar{\beta}\right)=0
$$

which implies

$$
\left(A_{\omega}(\alpha), \bar{\beta}\right)=\left(\left[\frac{d \alpha}{d t}(0)\right], \bar{\beta}\right)=\frac{i}{2} \int_{S} \frac{d \alpha}{d t}(0) \wedge \beta
$$

It is immediate from the definition of the Teichmüller deformation that

$$
\frac{d \omega}{d t}(0)=\bar{\omega}
$$


By writing $\alpha_{t}=\left(\frac{\alpha_{t}}{\omega_{t}}\right) \omega_{t}$ and differentiating, taking (2.14) into account, we derive the following formula:

$$
\frac{d \alpha}{d t}(0)=\left(\frac{d}{d t} \frac{\alpha_{t}}{\omega_{t}}(0)\right) \cdot \omega+\frac{\alpha}{\omega} \bar{\omega} .
$$

By formula (2.15), the 1 -form $d \alpha / d t(0)-(\alpha / \omega) \bar{\omega}$ is of type $(1,0)$, hence (taking into account that $\beta$ is holomorphic) we have

$$
\frac{i}{2} \int_{S} \frac{d \alpha}{d t}(0) \wedge \beta=\frac{i}{2} \int_{S} \frac{\alpha}{\omega} \bar{\omega} \wedge \beta=-B_{\omega}(\alpha, \beta) .
$$

The first formula in the statement follows from formulas (2.13) and (2.16).

Finally, let $\left\{\omega_{1}, \ldots, \omega_{g}\right\} \subset H^{1,0}(S)$ be any orthonormal basis (in the sense that $\left.\left(\omega_{i}, \omega_{j}\right)=\delta_{i j}\right)$. The system $\left\{\bar{\omega}_{1}, \ldots, \bar{\omega}_{g}\right\} \subset H^{0,1}(S)$ is pseudoorthonormal (in the sense that $\left(\bar{\omega}_{i}, \bar{\omega}_{j}\right)=-\delta_{i j}$ ), hence

$$
A_{\omega}(\alpha)=-\sum_{j=1}^{g}\left(A_{\omega}(\alpha), \bar{\omega}_{j}\right) \bar{\omega}_{j}=\sum_{j=1}^{g} B_{\omega}\left(\alpha, \omega_{j}\right) \bar{\omega}_{j} .
$$

Thus the second formula in the statement is proved.

Remark 2.1. We warn the reader that in general (unless $\alpha \in \mathbb{C} \cdot \omega$ )

$$
A_{\omega}(\alpha) \neq \frac{\alpha}{\omega} \bar{\omega} .
$$

In fact, the 1-form $A_{\omega}(\alpha)$ is closed by definition, while the 1-form $(\alpha / \omega) \bar{\omega}$ is in general not closed. In order to compute $A_{\omega}(\alpha)$ directly, it is necessary to consider the appropriate projection of the 1 -form $(\alpha / \omega) \bar{\omega}$ onto the subspace of closed 1-forms. We carry out such a direct calculation below, following Lemma 2.1 in [ [F2]. We stress that this calculation, although not needed for first variation formulas, is important for the correct derivation of second variation formulas along the Teichmüller flow.

Let $\partial$ and $\bar{\partial}$ denote respectively the type $(1,0)$ and the type $(0,1)$ exterior differentials on the Riemann surface $S$, defined as the projections of the (total) exterior differential $d$ on the subspaces of 1-forms of type $(1,0)$ and $(0,1)$ respectively. By definition, for all $v \in C^{\infty}(S)$, the 1 -form $\partial v$ is of type $(1,0)$, the 1 -form $\bar{\partial} v$ is of type $(0,1)$ and the following formula holds

$$
d v=\partial v+\bar{\partial} v .
$$

Note that the 1 -form $(\alpha / \omega) \bar{\omega}$ is $\bar{\partial}$-closed (but not $d$-closed, unless $\alpha \in \mathbb{C} \cdot \omega$ ), hence its $\bar{\partial}$-cohomology class has a unique anti-holomorphic representative $p_{\omega}(\alpha) \in H^{0,1}(S)$. In other words, there exist a unique anti-holomorphic 
form $p_{\omega}(\alpha) \in H^{0,1}(S)$ and a complex-valued function $v \in C^{\infty}(S)$ (unique up to additive constants) such that

$$
\frac{\alpha}{\omega} \bar{\omega}=p_{\omega}(\alpha)+\bar{\partial} v
$$

(the linear operator $p_{\omega}: H^{1,0}(S) \rightarrow H^{0,1}(S)$ is equivalent to the restriction to the subspace of meromorphic functions of the orthogonal projection from the space of square-integrable functions on $S$ onto the subspace of antimeromorphic functions, which appears in the formulas of [[F1] and [F2]).

Since $d \alpha / d t(0)$ and $p_{\omega}(\alpha)$ are closed forms and any closed form of type $(1,0)$ is holomorphic, by formulas (2.15) and (2.18) it follows that

$$
\frac{d \alpha}{d t}(0)-\left(p_{\omega}(\alpha)+d v\right) \in H^{1,0}(S)
$$

hence, by formulas (2.12) and (2.19), we conclude that the second fundamental form has the following expression:

$$
A_{\omega}(\alpha)=p_{\omega}(\alpha), \quad \text { for all } \alpha \in H^{1,0}(S) .
$$

In conclusion, the form $A_{\omega}(\alpha)$ is equal to $(\alpha / \omega) \bar{\omega}$ only up to a $\bar{\partial}$-exact correction term. If such a correction term were identically zero, the theory of the Kontsevich-Zorich cocycle would be much simpler!

The second fundamental form of the Hodge bundle is related to the derivative of the period matrix along the Teichmüller flow. We recall the definition of the period matrix. Let $\left\{a_{1}, \ldots, a_{g}, b_{1}, \ldots, b_{g}\right\}$ be a canonical basis of the first homology group $H_{1}(S, \mathbb{R})$ of a Riemann surface $S$ and let $\left\{\theta_{1}, \ldots, \theta_{g}\right\} \subset H^{1,0}(S)$ be the dual basis of holomorphic 1-forms, that is, the unique basis with the property that

$$
\theta_{i}\left(a_{j}\right)=\delta_{i j}, \quad \text { for all } i, j \in\{1, \ldots, g\} .
$$

The period matrix $\Pi_{i j}(S)$ is the $g \times g$ complex symmetric matrix with positive-definite imaginary part defined as follows:

$$
\Pi_{i j}(S):=\theta_{i}\left(b_{j}\right) \quad \text { for all } i, j \in\{1, \ldots, g\} .
$$

Lemma 2.2. Let $\mathcal{L}$ denote the Lie derivative along the Teichmüller flow on the space of Abelian differentials. The following formula holds:

$$
\mathcal{L} \prod_{i j}(S, \omega)=B_{\omega}\left(\theta_{i}, \theta_{j}\right), \quad \text { for all } i, j \in\{1, \ldots, g\} .
$$

Proof. By Rauch's formula (see for instance [IT], Prop. A.3), for any Beltrami differential $\mu$ on $S$, we have

$$
\frac{d \Pi_{i j}}{d \mu}(S)=\frac{i}{2} \int_{S} \theta_{i} \theta_{j} \mu, \quad \text { for all } i, j \in\{1, \ldots, g\} .
$$


By definition, at any holomorphic quadratic differential $q=\omega^{2}$ on $S$ the Teichmüller flow is tangent to the equivalence class of Beltrami differentials represented by the Beltrami differential

$$
\mu=\frac{|q|}{q}=\frac{\bar{\omega}}{\omega} .
$$

The statement then follows immediately from Rauch's formula.

For any $\omega \in \mathcal{H}_{g}^{(1)}$ (that is, for any Abelian differential $\omega \in \mathcal{H}_{g}$ of unit total area, see formula (2.2) $)$, the second fundamental form $B_{\omega}$ satisfies a uniform upper bound and a spectral gap bound, proved below.

Lemma 2.3. For any Abelian differential $\omega \in \mathcal{H}_{g}^{(1)}$ on a Riemann surface $S$, the following uniform bound holds:

$$
\left\|B_{\omega}\right\|:=\max \left\{\frac{\left|B_{\omega}(\alpha, \beta)\right|}{\|\alpha\|\|\beta\|}: \alpha, \beta \in H^{1,0}(S) \backslash\{0\}\right\}=1,
$$

and the maximum is achieved at $(\alpha, \beta)=(\omega, \omega)$, in fact we have

$$
B_{\omega}(\omega, \omega)=\|\omega\|^{2}=1 \text {. }
$$

Let $\langle\omega\rangle^{\perp} \subset H^{1,0}(S)$ be Hodge orthogonal complement of the complex line $\langle\omega\rangle=\mathbb{C} \cdot \omega$. The following spectral gap bound holds:

(2.25) $\max \left\{\frac{\left|B_{\omega}(\alpha, \beta)\right|}{\|\alpha\|\|\beta\|}: \alpha, \beta \in H^{1,0}(S) \backslash\{0\}\right.$ and $\left.\alpha \in\langle\omega\rangle^{\perp}\right\}<1$.

Proof. By the Cauchy-Schwarz inequality in the space $L^{2}\left(S, \frac{i}{2} \omega \wedge \bar{\omega}\right)$, it follows that, for all $\alpha, \beta \in H^{1,0}(S)$,

$$
\begin{aligned}
\left|B_{\omega}(\alpha, \beta)\right| & =\left|\frac{i}{2} \int_{S} \frac{\alpha}{\omega} \frac{\beta}{\omega} \omega \wedge \bar{\omega}\right| \\
& \leq\left(\frac{i}{2} \int_{S}\left|\frac{\alpha}{\omega}\right|^{2} \omega \wedge \bar{\omega}\right)^{1 / 2}\left(\frac{i}{2} \int_{S}\left|\frac{\beta}{\omega}\right|^{2} \omega \wedge \bar{\omega}\right)^{1 / 2} \\
& =\left(\frac{i}{2} \int_{S} \alpha \wedge \bar{\alpha}\right)^{1 / 2}\left(\frac{i}{2} \int_{S} \beta \wedge \bar{\beta}\right)^{1 / 2}=\|\alpha\|\|\beta\| .
\end{aligned}
$$

The uniform upper bound in formula (2.23) is therefore proved. The bound is achieved at $(\omega, \omega)$ since it is immediate by the definition that

$$
B_{\omega}(\omega, \omega)=\|\omega\|^{2} .
$$

In fact, $\|\omega\|^{2}$ is by definition equal to the area of surface $S$ with respect to the flat metric associated to the Abelian differential $\omega \in \mathcal{H}_{g}^{(1)}$, which, by definition of $\mathcal{H}_{g}^{(1)}$, is normalized (equal to 1 ). 
The spectral gap bound in formula (2.25) is proved as follows. By a fundamental property of the Cauchy-Schwarz inequality, equality holds in formula (2.26) if and only if there exists a constant const $\in \mathbb{C}$ such that

$$
\frac{\alpha}{\omega}=\operatorname{const} \cdot \frac{\bar{\beta}}{\bar{\omega}} .
$$

The functions $\alpha / \omega$ and $\beta / \omega$ are meromorphic on the Riemann surface $S$, hence $\bar{\beta} / \bar{\omega}$ is anti-meromorphic. Since the only meromorphic functions which are also anti-meromorphic are the constant functions, if follows that equality holds in the Cauchy-Schwarz inequality if and only if $\alpha$ and $\beta$ belong to the complex line $\langle\omega\rangle \subset H^{1,0}(S)$. Thus, if $\alpha \in\langle\omega\rangle^{\perp} \backslash\{0\}$, the Cauchy-Schwarz inequality is strict and the spectral gap bound stated above on the second fundamental form $B_{\omega}$ is proved.

The curvature form of the Hodge bundle appears in the second variation formulas for the Hodge norm computed in [F2], $\S 2-5$, which we will recall in the next section. For consistence with the notations of that paper, we adopt below a sign convention for the curvature matrix which is opposite to that of formula (2.10). For any Abelian differential $\omega \in \mathcal{H}_{g}$, let $H_{\omega}$ be the Hermitian curvature form defined as follows: for all $\alpha, \beta \in H^{1,0}(S)$,

$$
H_{\omega}(\alpha, \beta)=-\left(A_{\omega}(\alpha), A_{\omega}(\beta)\right)=\left(A_{\omega}^{*} A_{\omega}(\alpha), \beta\right) .
$$

It follows from Lemma 2.3 that, for any Abelian differential $\omega \in \mathcal{H}_{g}^{(1)}$ on a Riemann surface $S$, the second fundamental form operator (the KodairaSpencer map) $A_{\omega}: H^{1,0}(S) \rightarrow H^{0,1}(S)$ is a contraction with respect to the Hodge norm and, as a consequence, the Hermitian positive semi-definite curvature form $H_{\omega}$ is uniformly bounded. In fact, the following result holds:

Lemma 2.4. For any Abelian differential $\omega \in \mathcal{H}_{g}^{(1)}$ on a Riemann surface $S$, the following bounds hold:

$$
\begin{aligned}
& \left\|A_{\omega}\right\|:=\max \left\{\frac{\left\|A_{\omega}(\alpha)\right\|}{\|\alpha\|}: \alpha \in H^{1,0}(S) \backslash\{0\}\right\}=1 \\
& \left\|H_{\omega}\right\|:=\max \left\{\frac{\left|H_{\omega}(\alpha, \beta)\right|}{\|\alpha\|\|\beta\|}: \alpha, \beta \in H^{1,0}(S) \backslash\{0\}\right\}=1
\end{aligned}
$$

and the maximum is achieved at $(\alpha, \beta)=(\omega, \omega)$, in fact we have

$$
A_{\omega}(\omega)=\bar{\omega} \quad \text { and } \quad H_{\omega}(\omega, \omega)=\|\omega\|^{2}=1 .
$$

The following spectral gap result holds:

$$
\begin{aligned}
& \max \left\{\frac{\left\|A_{\omega}(\alpha)\right\|}{\|\alpha\|}: \alpha \in\langle\omega\rangle^{\perp} \backslash\{0\} \subset H^{1,0}(S)\right\}<1 ; \\
& \max \left\{\frac{\left|H_{\omega}(\alpha, \beta)\right|}{\|\alpha\|\|\beta\|}: \alpha, \beta \in H^{1,0}(S) \backslash\{0\} \text { and } \alpha \in\langle\omega\rangle^{\perp}\right\}<1 .
\end{aligned}
$$


Proof. By Lemma 2.1, it follows that, for all $\alpha \in H^{1,0}(S)$, we have

$$
\left\|A_{\omega}(\alpha)\right\|=\max _{\beta \neq 0} \frac{\left|\left(A_{\omega}(\alpha), \bar{\beta}\right)\right|}{\|\beta\|}=\max _{\beta \neq 0} \frac{\left|B_{\omega}(\alpha, \beta)\right|}{\|\beta\|},
$$

and by the definition of the curvature form $H_{\omega}=A_{\omega}^{*} \cdot A_{\omega}$, we also have

$$
\left|H_{\omega}(\alpha, \beta)\right|=\left|\left(A_{\omega}(\alpha), A_{\omega}(\beta)\right)\right| \leq\left\|A_{\omega}(\alpha)\right\|\left\|A_{\omega}(\beta)\right\| .
$$

The upper bounds in formulas (2.28) and (2.30) therefore follow from the corresponding results for the form $B_{\omega}$ established in Lemma 2.3.

The identities (2.29) follow from formula (2.24) in Lemma 2.3, which states that $B_{\omega}(\omega, \omega)=1$. In fact, by Lemma 2.1 we have

$$
A_{\omega}(\omega)=B_{\omega}(\omega, \omega) \bar{\omega}=\bar{\omega} .
$$

Finally, by the definition of the curvature form it follows that

$$
H_{\omega}(\omega, \omega)=-\left(A_{\omega}(\omega), A_{\omega}(\omega)\right)=-(\bar{\omega}, \bar{\omega})=\|\omega\|^{2}=1 .
$$

The argument is complete.

For any Abelian differential $\omega \in \mathcal{H}_{g}$ on a Riemann surface $S$ the matrix $H$ of the Hermitian curvature form $H_{\omega}$ with respect to any Hodgeorthonormal basis $\Omega:=\left\{\omega_{1}, \ldots, \omega_{g}\right\}$ can be written as follows.

Let $B$ be the matrix of the bilinear form $B_{\omega}$ on $H^{1,0}(S)$ with respect to the basis $\Omega$, that is:

$$
B_{j k}:=\frac{i}{2} \int_{S} \frac{\omega_{j} \omega_{k}}{\omega} \bar{\omega} .
$$

By formula (2.10) and Lemma 2.1, the matrix $H$ of the Hermitian curvature form $H_{\omega}$ of the vector bundle $H^{1,0}$ over $\omega \in \mathcal{H}_{g}$ in the orthonormal basis $\Omega$ can be written as follows:

$$
H=B \cdot B^{*} .
$$

(The above formula is the corrected version of the formula $H=B^{*} B$ which appears as formula (4.3) in [F2] and as formula (44) in [F3]. This mistake there is of no consequence). In particular, since the form $B_{\omega}$ is symmetric, the forms $H_{\omega}$ and $B_{\omega}$ have the same rank and their eigenvalues are related. Let $\operatorname{EV}\left(H_{\omega}\right)$ and $\operatorname{EV}\left(B_{\omega}\right)$ denote the set of eigenvalues of the forms $H_{\omega}$ and $B_{\omega}$ respectively. The following identity holds:

$$
\operatorname{EV}\left(H_{\omega}\right)=\left\{|\lambda|^{2} \mid \lambda \in \operatorname{EV}\left(B_{\omega}\right)\right\} .
$$

For every Abelian differential $\omega \in \mathcal{H}_{g}^{(1)}$, the eigenvalues of the positivesemidefinite form $H_{\omega}$ on $H^{1,0}(S)$ will be denoted as follows:

$$
\Lambda_{1}(\omega) \equiv 1>\Lambda_{2}(\omega) \geq \cdots \geq \Lambda_{g}(\omega) \geq 0 .
$$

We remark that the top eigenvalue $\Lambda_{1}(\omega)$ is equal to 1 and the second eigenvalue $\Lambda_{2}(\omega)<1$ for any Abelian differentials $\omega \in \mathcal{H}_{g}^{(1)}$ as a consequence 
of Lemma 2.4, in particular all of the above eigenvalues give well-defined, continuous, non-negative, bounded functions on the moduli space $\mathcal{H}_{g}^{(1)}$ of all (normalized) Abelian differentials.

By the Hodge representation theorem for Riemann surfaces, the forms $H_{\omega}$ and $B_{\omega}$ induce complex-valued bilinear forms $H_{\omega}^{\mathbb{R}}$ and $B_{\omega}^{\mathbb{R}}$ on the real cohomology $H^{1}(S, \mathbb{R})$ : for all $c_{1}, c_{2} \in H^{1}(S, \mathbb{R})$,

$$
\begin{aligned}
& H_{\omega}^{\mathbb{R}}\left(c_{1}, c_{2}\right):=H_{\omega}\left(h\left(c_{1}\right), h\left(c_{2}\right)\right) \\
& B_{\omega}^{\mathbb{R}}\left(c_{1}, c_{2}\right):=B_{\omega}\left(h\left(c_{1}\right), h\left(c_{2}\right)\right) .
\end{aligned}
$$

The forms $H_{\omega}^{\mathbb{R}}$ and $B_{\omega}^{\mathbb{R}}$ on $H^{1}(S, \mathbb{R})$ have the same rank, which is equal to twice the common rank of the forms $H_{\omega}$ and $B_{\omega}$ on $H^{1,0}(S)$. The bilinear form $H_{\omega}^{\mathbb{R}}$ induces a real-valued, positive semi-definite quadratic form, while the quadratic form induced by the bilinear form $B_{\omega}^{\mathbb{R}}$ is complex-valued.

2.6. Variational formulas for the Hodge norm. We recall below some basic variational formulas from [ [F2], $2, \S 3$ and $\S 5$, reformulated in geometric terms. Such formulas generalize the fundamental Kontsevich formula for the sum of all non-negative Lyapunov exponents of the Hodge bundle $[\mathrm{K}]$.

2.6.1. First Variation. The second fundamental form of the Hodge bundle measures the first variation of the Hodge norm along a parallel (locally constant) section for the Gauss-Manin connection. In fact, the formula given below (implicit in the computation of [F2], §2) holds. Let $(S, \omega)$ be a pair (Riemann surface $S$, holomorphic 1-form $\omega$ on $S$ ). For any cohomology class $c \in H^{1}(S, \mathbb{R})$, let $h_{\omega}(c)$ be the unique holomorphic 1-form such that $c$ is the cohomology class of the closed 1-form $\operatorname{Re} h_{\omega}(c)$ in the de Rham cohomology $H_{\text {deRham }}^{1}(S, \mathbb{R})$. We remark that for any given $c \in H^{1}(S, \mathbb{R})$ the holomorphic 1-form $h_{\omega}(c)$ only depends on the Riemann surface $S$. However, the Riemann surface $S$ underlying a given holomorphic 1-form $\omega$ is unique and it will be convenient to write below the harmonic representative as a function of the holomorphic 1-form $\omega$ on $S$.

Lemma 2.5. The Lie derivative $\mathcal{L}$ of the Hodge inner product $\left(c_{1}, c_{2}\right)_{\omega}$ of parallel (locally constant) sections $c_{1}, c_{2} \in H^{1}(S, \mathbb{R})$ in the direction of the Teichmüller flow can be written as follows:

$$
\mathcal{L}\left(c_{1}, c_{2}\right)_{\omega}=2 \operatorname{Re}\left(A_{\omega}\left(h_{\omega}\left(c_{1}\right)\right), \overline{h_{\omega}\left(c_{2}\right)}\right) .
$$

Proof. The argument is just a rephrasing of the proof of Lemma 2.1' of [F2] in the language of differential geometry. Let us recall that by definition, for any pair $c_{1}, c_{2} \in H^{1}(S, \mathbb{R})$, the Hodge inner product is defined as

$$
\left(c_{1}, c_{2}\right)_{\omega}=\operatorname{Re}\left(h_{\omega}\left(c_{1}\right), h_{\omega}\left(c_{2}\right)\right) .
$$


Since the Gauss-Manin connection is compatible with the Hermitian intersection form, we can compute

$$
\begin{aligned}
\mathcal{L}\left(c_{1}, c_{2}\right)_{\omega} & =\operatorname{Re}\left(D_{H_{\mathbb{C}}^{1}} h_{\omega}\left(c_{1}\right), h_{\omega}\left(c_{2}\right)\right) \\
& +\operatorname{Re}\left(h_{\omega}\left(c_{1}\right), D_{H_{\mathbb{C}}^{1}} h_{\omega}\left(c_{2}\right)\right),
\end{aligned}
$$

and, since $\left(h_{\omega}\left(c_{1}\right), \overline{h_{\omega}\left(c_{2}\right)}\right)=0$, we also have

$$
\left(D_{H_{\mathbb{C}}^{1}} h_{\omega}\left(c_{1}\right), \overline{h_{\omega}\left(c_{2}\right)}\right)+\left(h_{\omega}\left(c_{1}\right), D_{H_{\mathbb{C}}^{1}} \overline{h_{\omega}\left(c_{2}\right)}\right)=0 .
$$

Any cohomology class $c \in H^{1}(S, \mathbb{R})$ can be interpreted as a parallel (constant) local section of the bundle $H_{\mathbb{C}}^{1}$. Since by definition of the differential $h_{\omega}(c) \in H^{1,0}(S)$ we have $c=\left[h_{\omega}(c)+\overline{h_{\omega}(c)}\right] / 2$ and since the Gauss-Manin connection is real (on real tangent vectors of the moduli space it commutes with the complex conjugation), it follows that

$$
D_{H_{\mathbb{C}}^{1}} h_{\omega}(c)=-D_{H_{\mathbb{C}}^{1}} \overline{h_{\omega}(c)}=-\overline{D_{H_{\mathbb{C}}^{1}} h_{\omega}(c)} .
$$

From formulas (2.36) and (2.37) we can derive the identities

$$
\begin{aligned}
\left(D_{H_{\mathbb{C}}^{1}} h_{\omega}\left(c_{1}\right), h_{\omega}\left(c_{2}\right)\right) & =-\left(\overline{D_{H_{\mathbb{C}}^{1}} h_{\omega}\left(c_{1}\right)}, h_{\omega}\left(c_{2}\right)\right)=\overline{\left(D_{H_{\mathbb{C}}^{1}} h_{\omega}\left(c_{1}\right), \overline{h_{\omega}\left(c_{2}\right)}\right)} \\
\left(h_{\omega}\left(c_{1}\right), D_{H_{\mathbb{C}}^{1}} h_{\omega}\left(c_{2}\right)\right) & =-\left(h_{\omega}\left(c_{1}\right), \overline{D_{H_{\mathbb{C}}^{1}} h_{\omega}\left(c_{2}\right)}\right)=\left(D_{H_{\mathbb{C}}^{1}} h_{\omega}\left(c_{1}\right), \overline{h_{\omega}\left(c_{2}\right)}\right) .
\end{aligned}
$$

In conclusion, from formula (2.35), by the above identities and by the definition (2.7) of the second fundamental form, it follows that

$$
\begin{aligned}
\mathcal{L}\left(c_{1}, c_{2}\right)_{\omega} & =2 \operatorname{Re}\left(D_{H_{\mathbb{C}}^{1}} h_{\omega}\left(c_{1}\right), \overline{h_{\omega}\left(c_{2}\right)}\right) \\
& =2 \operatorname{Re}\left(\left(I-\pi_{1}\right) D_{H_{\mathbb{C}}^{1}} h_{\omega}\left(c_{1}\right), \overline{h_{\omega}\left(c_{2}\right)}\right) \\
& =2 \operatorname{Re}\left(A_{\omega}\left(h_{\omega}\left(c_{1}\right)\right), \overline{h_{\omega}\left(c_{2}\right)}\right) .
\end{aligned}
$$

The stated first variation formula is therefore proved.

The fundamental variational formula, computed in [F2], Lemma 2.1', for the Lie derivative of the Hodge norm of a parallel (locally constant) section $c \in H^{1}(S, \mathbb{R})$ in the direction of the Teichmüller flow can now be derived from Lemma 2.5 and Lemma 2.1.

Lemma 2.6. The following variational formula holds:

$$
\mathcal{L}\left(c_{1}, c_{2}\right)_{\omega}=-2 \operatorname{Re} B_{\omega}\left(h_{\omega}\left(c_{1}\right), h_{\omega}\left(c_{2}\right)\right)=-2 \operatorname{Re} B_{\omega}^{\mathbb{R}}\left(c_{1}, c_{2}\right) .
$$

Remark 2.2. Lemma 2.1 can also be derived from the variational formulas of Lemma 2.5 (proved above) and Lemma 2.6 (proved as part of Lemma 
$2.1^{\prime}$ in $\left.[\overline{\mathrm{F} 2}]\right)$. In fact, by comparison of the variational formulas of Lemmas 2.5 and 2.6, for any cohomology classes $c_{1}, c_{2} \in H^{1}(S, \mathbb{R})$,

$$
\operatorname{Re}\left(A_{\omega}\left(h_{\omega}\left(c_{1}\right)\right), \overline{h_{\omega}\left(c_{2}\right)}\right)=-\operatorname{Re} B_{\omega}\left(h_{\omega}\left(c_{1}\right), h_{\omega}\left(c_{2}\right)\right),
$$

which implies the main identity of Lemma 2.1 since the operator $A_{\omega}$ is complex linear, the intersection form is Hermitian by definition and the form $B_{\omega}$ is complex bilinear.

The variational formula of Lemma 2.6 implies a uniform bound and a spectral gap result on the exponential growth of Hodge norms based on the uniform bound and on the spectral gap estimate of Lemma 2.3 (see [F2], Lemma 2.1' and Corollary 2.2).

Let $\Lambda: \mathcal{H}_{g}^{(1)} \rightarrow \mathbb{R}^{+}$be the function defined as follows: for all $\omega \in \mathcal{H}_{g}^{(1)}$,

$$
\Lambda(\omega):=\max \left\{\frac{\left|B_{\omega}(\alpha, \alpha)\right|}{\|\alpha\|^{2}}: \alpha \in\langle\omega\rangle^{\perp} \backslash\{0\} \subset H^{1,0}(S)\right\} .
$$

By definition $\Lambda$ is a continuous function on the moduli space of normalized (unit area) Abelian differentials and by Lemma2.3 it is everywhere strictly less than 1 , hence it achieves its maximum (strictly less than 1) on every compact subset. It is proved in [[F2] that $\Lambda$ has supremum equal to 1 on every connected component of every stratum of the moduli space.

For any Abelian differential $\omega \in \mathcal{H}_{g}$ on a Riemann surface $S$, let $\|c\|_{\omega}$ denote the Hodge norm of a real cohomology class $c \in H^{1}(S, \mathbb{R})$, that is, the Hodge norm of the holomorphic 1-form $h_{\omega}(c) \in H^{1,0}(S)$.

Corollary 2.1. The Lie derivative of the Hodge norm along the Teichmüller flow admits the following bounds: for any Abelian differential $\omega \in \mathcal{H}_{g}^{(1)}$ on a Riemann surface $S$ and for any cohomology class $c \in H^{1}(S, \mathbb{R})$,

$$
\left|\mathcal{L} \log \|c\|_{\omega}\right| \leq 1
$$

for any cohomology class $c \in\langle[\operatorname{Re}(\omega)],[\operatorname{Im}(\omega)]\rangle^{\perp}$,

$$
\left|\mathcal{L} \log \|c\|_{\omega}\right| \leq \Lambda(\omega)<1 .
$$

Proof. By Lemma 2.6 for any Abelian differential $\omega \in \mathcal{H}_{g}$ on a Riemann surface $S$ and for any cohomology class $c \in H^{1}(S, \mathbb{R})$ we have

$$
\mathcal{L} \log \|c\|_{\omega}=-\frac{\operatorname{Re} B_{\omega}^{\mathbb{R}}(c, c)}{\|c\|_{\omega}^{2}} ;
$$

hence the statement follows from Lemma 2.3. In fact, for any cohomology class $c \in H^{1}(S, \mathbb{R})$, the Abelian differential $h_{\omega}(c)$ belongs to the Hodge orthogonal complemement $\langle\omega\rangle^{\perp} \subset H^{1,0}(S)$ if and only if $c$ belongs to the Hodge orthogonal complemement $\langle[\operatorname{Re}(\omega)],[\operatorname{Im}(\omega)]\rangle^{\perp} \subset H^{1}(S, \mathbb{R})$. 
The above universal bound and spectral gap estimate immediately extends to all exterior powers of the Hodge bundle. For every Abelian differential $\omega \in \mathcal{H}_{g}$ on a Riemann surface $S$ and for every $k \in\{1, \ldots, 2 g\}$, the Hodge norm $\|\cdot\|_{\omega}$ on $H^{1}(S, \mathbb{R})$ induces a natural norm (also called the Hodge norm) $\left\|c_{1} \wedge \cdots \wedge c_{k}\right\|_{\omega}$ on polyvectors $c_{1} \wedge \cdots \wedge c_{k} \in \Lambda^{k}\left(H^{1}(S, \mathbb{R})\right)$.

Corollary 2.2. The Lie derivative of the Hodge norm along the Teichmüller flow admits the following bounds: for any Abelian differential $\omega \in \mathcal{H}_{g}^{(1)}$ on a Riemann surface $S$, for any $k \in\{1, \ldots, g\}$ and for any polyvector $c_{1} \wedge \cdots \wedge c_{k} \in \Lambda^{k}\left(H^{1}(S, \mathbb{R})\right)$ such that the span $\left\langle c_{1}, \ldots, c_{k}\right\rangle \subset H^{1}(S, \mathbb{R})$ is an isotropic subspace, we have:

$$
\left|\mathcal{L} \log \left\|c_{1} \wedge \cdots \wedge c_{k}\right\|_{\omega}\right| \leq k
$$

for any $k \geq 2$ the following stronger bound holds:

$$
\left|\mathcal{L} \log \left\|c_{1} \wedge \cdots \wedge c_{k}\right\|_{\omega}\right| \leq 1+(k-1) \Lambda(\omega)<k ;
$$

2.6.2. Second Variation. The $\operatorname{SL}(2, \mathbb{R})$-orbit of any holomorphic Abelian differential $\omega_{0} \in \mathcal{H}_{g}$ is isomorphic to the unit tangent bundle of a hyperbolic surface (generically a copy of the Poincaré disk). Thus the left quotient $\mathrm{SO}(2, \mathbb{R}) \backslash\left(\mathrm{SL}(2, \mathbb{R}) \omega_{0}\right)$ is a hyperbolic surface, called a Teichmüller disk.

There is a natural action of $\mathbb{C}^{*}$ on the space $\mathcal{H}_{g}$ by multiplication of Abelian differentials by nonzero complex numbers. The corresponding projectivization $\mathrm{PH}_{g}:=\mathcal{H}_{g} / \mathbb{C}^{*}$ is foliated by Teichmüller disks endowed with the hyperbolic metric. We remark that for consistency with a standard normalization for the Teichmüller geodesic flow adopted in the literature the hyperbolic metric is normalized to have curvature equal to -4 . We have the following basic variational formula for the leafwise hyperbolic Laplacian $\triangle$ of the Hodge norm $\|c\|_{\omega}$ at a "point" $\omega$ of the projectivized moduli space $\mathrm{PH}_{g}$ (see [F2], Lemmas 2.1' and 3.2, [F3], Lemma 4.3):

Lemma 2.7. The following variational formula for the Hodge norm holds:

$$
\triangle \log \|c\|_{\omega}=4 \frac{H_{\omega}^{\mathbb{R}}(c, c)}{\|c\|_{\omega}^{2}}-2 \frac{\left|B_{\omega}^{\mathbb{R}}(c, c)\right|^{2}}{\|c\|_{\omega}^{4}} \geq 0 .
$$

Remark 2.3. In fact, given a cohomology class $c$ in $H^{1}(S, \mathbb{R})$, the Hodge norm $\|c\|_{\omega}$ at a point $(S, \omega)$ of $\mathcal{H}_{g}$ is completely determined by the complex structure of the underlying Riemann surface $S$. Thus, for any holomorphic form $\omega^{\prime}=$ const $\cdot \omega$ one has $\|c\|_{\omega}=\|c\|_{\omega^{\prime}}$. Whenever, in addition const $=\exp (i x)$ with real $x$, then $H_{\omega}=H_{\omega^{\prime}}$ and $\left|B_{\omega}\right|=\left|B_{\omega^{\prime}}\right|$. Thus, all the quantities in the above formula are $\mathrm{SO}(2, \mathbb{R})$-invariant, which makes it legitimate to consider them defined on a Teichmüller disk in the projectivized moduli space $\mathrm{P} \mathcal{H}_{g}$. It will often be convenient to pull back to $\mathcal{H}_{g}$ the functions defined on the projectivized moduli space $\mathrm{P} \mathcal{H}_{g}$. When operating 
with the leafwise hyperbolic Laplacian $\triangle$, we will always tacitly verify the $\mathrm{SO}(2, \mathbb{R})$-invariance of the functions involved.

2.7. Variational formulas for exterior powers. The above variational formulas can be generalized to all the exterior powers of the KontsevichZorich cocycle. For any $k \in\{1, \ldots, g\}$, let us denote by $G_{k}\left(H_{\mathbb{R}}^{1}\right)$ the total space of the Grassmannian bundle of isotropic $k$-dimensional subspaces of the Hodge bundle. By definition, the fiber $G_{k}\left(H_{\mathbb{R}}^{1}\right)_{\omega}$ of the bundle $G_{k}\left(H_{\mathbb{R}}^{1}\right)$ at any Abelian differential $\omega \in \mathcal{H}_{g}$ on a Riemann surface $S$ is the Grassmannian manifold of all $k$-dimensional isotropic subspaces of the symplectic vector space $H^{1}(S, \mathbb{R})$.

Let $\Phi_{k}$ denote the function on the Grassmannian bundle $G_{k}\left(H_{\mathbb{R}}^{1}\right)$ defined as follows (see [ [F2], §5). Let $\omega \in \mathcal{H}_{g}$ be an Abelian differential on a Riemann surface $S$ and let $I_{k} \subset H^{1}(S, \mathbb{R})$ be any isotropic subspace of dimension $k \in\{1, \ldots, g\}$. Let $\left\{c_{1}, \ldots, c_{k}\right\} \subset I_{k}$ be any Hodgeorthonormal basis and let $\left\{c_{1}, \ldots, c_{k}, c_{k+1}, \ldots, c_{g}\right\} \subset H^{1}(S, \mathbb{R})$ be any Hodge-orthonormal Lagrangian completion. Let

$$
\Phi_{k}\left(\omega, I_{k}\right):=2 \sum_{i=1}^{k} H_{\omega}^{\mathbb{R}}\left(c_{i}, c_{i}\right)-\sum_{i, j=1}^{k}\left|B_{\omega}^{\mathbb{R}}\left(c_{i}, c_{j}\right)\right|^{2}
$$

Lemma 2.8 (Forni ["[F2], Lemma 5.2'). The function $\Phi_{k}\left(\omega, I_{k}\right)$ depends only on $\omega \in \mathcal{H}_{g}$ and on the isotropic subspace $I_{k} \subset H^{1}(S, \mathbb{R})$ and is independent of the choice of the orthonormal basis $\left\{c_{1}, \ldots, c_{k}\right\} \subset I_{k}$ and of its Hodge-orthonormal Lagrangian completion $\left\{c_{1}, \ldots, c_{k}, c_{k+1}, \ldots, c_{g}\right\}$. It can be also expressed as

$$
\Phi_{k}\left(\omega, I_{k}\right)=\sum_{i=1}^{g} \Lambda_{i}(\omega)-\sum_{i, j=k+1}^{g}\left|B_{\omega}^{\mathbb{R}}\left(c_{i}, c_{j}\right)\right|^{2}
$$

(for $k=g$ the second sum on the right hand side is defined to be null). For any normalized (unit area) Abelian differential $\omega \in \mathcal{H}_{g}^{(1)}$ on a surface $S$ and for any $k$-dimensional isotropic subspace $I_{k} \subset H^{1}(S, \mathbb{R})$, the following bound holds:

$$
\left|\Phi_{k}\left(\omega, I_{k}\right)\right| \leq \min (2 k, g) \quad \text { and the inequality is strict for } k \geq 2 \text {. }
$$

Proof. We reproduce here the proof of equivalence of definitions (2.46) and (2.47) since the same kind of calculations will be repeatedly used in the sequel. Let $\left\{\omega_{1}, \ldots, \omega_{g}\right\} \subset H^{1,0}(S)$ be an orthonormal basis of Abelian differentials representing the orthonormal basis $\left\{c_{1}, \ldots, c_{g}\right\} \subset H^{1}(S, \mathbb{R})$. By the definition of $H_{\omega}^{\mathbb{R}}$ and $B_{\omega}^{\mathbb{R}}$ we have that $H_{\omega}^{\mathbb{R}}\left(c_{i}, c_{j}\right):=H_{\omega}\left(\omega_{i}, \omega_{j}\right)$ and $B_{\omega}^{\mathbb{R}}\left(c_{i}, c_{j}\right):=B_{\omega}\left(\omega_{i}, \omega_{j}\right)$. By formula 2.31) we also have the relation 
$H_{\omega}=B_{\omega} \cdot B_{\omega}^{*}$, which implies that

$$
H_{\omega}\left(\omega_{i}, \omega_{i}\right)=\sum_{j=1}^{g} B_{\omega}\left(\omega_{i}, \omega_{j}\right) \overline{B_{\omega}\left(\omega_{i}, \omega_{j}\right)}=\sum_{j=1}^{g}\left|B_{\omega}\left(\omega_{i}, \omega_{j}\right)\right|^{2} .
$$

By definition 2.32 one has

$$
\Lambda_{1}(\omega)+\cdots+\Lambda_{g}(\omega)=\operatorname{Tr} H_{\omega}=\sum_{i=1}^{g} \sum_{j=1}^{g}\left|B_{\omega}\left(\omega_{i}, \omega_{j}\right)\right|^{2} .
$$

By formulas (2.49) and (2.50) and by taking into account that $B$ is symmetric, we transform formula (2.46) as follows:

$$
\begin{aligned}
\Phi_{k}\left(\omega, I_{k}\right) & :=2 \sum_{i=1}^{k} H_{\omega}\left(\omega_{i}, \omega_{i}\right)-\sum_{i, j=1}^{k}\left|B_{\omega}\left(\omega_{i}, \omega_{j}\right)\right|^{2} \\
& =2 \sum_{i=1}^{k} \sum_{j=1}^{g}\left|B_{\omega}\left(\omega_{i}, \omega_{j}\right)\right|^{2}-\sum_{i, j=1}^{k}\left|B_{\omega}\left(\omega_{i}, \omega_{j}\right)\right|^{2} \\
& =\sum_{i=1}^{k} \sum_{j=1}^{g}\left|B_{\omega}\left(\omega_{i}, \omega_{j}\right)\right|^{2}+\sum_{i=1}^{k} \sum_{j=k+1}^{g}\left|B_{\omega}\left(\omega_{i}, \omega_{j}\right)\right|^{2} \\
& =\sum_{i, j=1}^{g}\left|B_{\omega}\left(\omega_{i}, \omega_{j}\right)\right|^{2}-\sum_{i, j=k+1}^{g}\left|B_{\omega}\left(\omega_{i}, \omega_{j}\right)\right|^{2} \\
& =\sum_{i=1}^{g} \Lambda_{i}(\omega)-\sum_{i, j=k+1}^{g}\left|B_{\omega}\left(\omega_{i}, \omega_{j}\right)\right|^{2} .
\end{aligned}
$$

Formula (2.47) is therefore established. Note that formula 2.46) does not depend on the choice of the system $\left\{c_{k+1}, \ldots, c_{g}\right\}$ in the orthonormal Lagrangian completion, while formula (2.47) does not depend on the choice of the orthonormal basis $\left\{c_{1}, \ldots, c_{k}\right\}$. Hence, the equality between formulas (2.46) and (2.47) proves that neither formula depends on the choice of the orthonormal basis of $I_{k}$ and of its orthonormal Lagrangian completion.

The bound in formula (2.48) follows from formulas (2.46) and (2.47), by taking into account that $H_{\omega}\left(\omega_{i}, \omega_{i}\right) \leq\left\|\omega_{i}\right\|^{2}=1$ and $\Lambda_{i}(\omega) \leq 1$, for all $i \in\{1, \ldots, g\}$, according to the upper bounds in formulas (2.28) and (2.32) respectively. Finally, by the spectral gap bound in Lemma 2.4 and by the consequent strict bound for the second curvature eigenvalue in formula (2.32), for $k \geq 2$ the inequality in formula (2.48) is strict .

The formulas below, computed in [[F2], Lemma 5.2 and 5.2', extend the formula in Lemma 2.7 to all exterior powers of the Hodge bundle. Let $\left\{c_{1}, \ldots, c_{k}\right\} \subset I_{k}$ be any Hodge-orthonormal basis of an isotropic subspace 
$I_{k} \subset H^{1}(S, \mathbb{R})$ on a Riemann surface $S$. Recall that the Euclidean structure defined by the Hodge scalar product on $H^{1}(S, \mathbb{R})$ defines the natural norm $\left\|c_{1} \wedge \cdots \wedge c_{k}\right\|_{\omega}$ of any polyvector which we also call the Hodge norm. Similarly to the case of the Hodge norm, it is defined only by the complex structure of the underlying Riemann surface. It follows from the definition (2.46) of $\Phi_{k}\left(\omega, I_{k}\right)$ that this function is $\mathrm{SO}(2, \mathbb{R})$-invariant (compare to Remark 2.3). Thus, for any $\left(S_{0}, \omega_{0}\right)$ in $\mathcal{H}_{g}$ the Hodge norm $\left\|c_{1} \wedge \cdots \wedge c_{k}\right\|_{\omega}$ defines a smooth function on the hyperbolic surface obtained as a left quotient $\mathrm{SO}(2, \mathbb{R}) \backslash \mathrm{SL}(2, \mathbb{R}) \cdot \omega_{0}$ of the orbit of $\omega_{0}$.

Lemma 2.9. For all $k \in\{1, \ldots, g\}$ the following formula holds:

$$
\triangle \log \left\|c_{1} \wedge \cdots \wedge c_{k}\right\|_{\omega}=2 \Phi_{k}\left(\omega, I_{k}\right) \geq 0 .
$$

Proof. See Lemma 5.2 and 5.2' in [F2].

Recall that our hyperbolic Laplacian $\triangle$ is written in the hyperbolic metric of constant curvature -4 . Any different choice of the constant negative curvature would change the RHS in the above formula by a constant factor.

For $k=1$ the formula of Lemma 2.9 reduces to that of Lemma 2.7

Remark 2.4. Note that for $k=g$ the Lagrangian subspace $I_{g}$ is not present in the right-hand side of definition (2.46) of $\Phi_{g}\left(\omega, I_{g}\right)$ :

$$
\Phi_{g}\left(\omega, I_{k}\right):=\sum_{i=1}^{g} \Lambda_{i}(\omega) .
$$

Thus, the function $\Phi_{g}$ is the pull-back to the Grassmannian bundle of Lagrangian subspaces of a function on the moduli space $\mathcal{H}_{g}$. Moreover, by definition (2.32) the above sum is the trace of the Hermitian form $H_{\omega}$, hence it is by definition the curvature of the Hermitian bundle $H^{1,0}$. This fundamental fact discovered in $[\mathrm{K}]$ is crucial for the validity of the Kontsevich formula for the sum of exponents. A version of this formula is stated in Corollary 3.3 below.

\section{The KontseviCH-ZORICH EXPONENTS}

In this section we derive formulas for the Lyapunov exponents of the Kontsevich-Zorich cocycle on the Hodge bundle in terms of the second fundamental form and curvature of the Hodge bundle.

3.1. Lyapunov exponents. Let $\left(T_{t}\right)_{t \in \mathbb{R}}: X \rightarrow X$ be a flow preserving a Borel ergodic probability measure $\mu$ on a locally compact topological space $X$. Let $\pi: M \rightarrow X$ be a real or complex $d$-dimensional vector bundle. In other words, the fiber $M_{x}:=\pi^{-1}(x)$ of the vector bundle above any $x \in X$ is a real or complex vector space isomorphic to $\mathbb{R}^{d}$ or $\mathbb{C}^{d}$ respectively. A real 
or complex linear cocycle $\left(F_{t}\right)_{t \in \mathbb{R}}: M \rightarrow M$ over the flow $\left(T_{t}\right)$ is a flow on the total space $M$ of the vector bundle such that, for all $(x, t) \in X \times \mathbb{R}$, the map $F_{t}: M_{x} \rightarrow M_{T_{t}(x)}$ is well-defined and linear over $\mathbb{R}$ or $\mathbb{C}$ respectively. Suppose that there exists a family of norms $\left\{|\cdot|_{x}\right\}_{x \in X}$ on the fibers of the vector bundle and, for all $(x, t) \in X \times \mathbb{R}$, let $\left\|F_{t}\right\|_{x}$ denote the operator norm of the linear map $F_{t}$ with respect to the norm $|\cdot|_{x}$ on $M_{x}$ and $|\cdot|_{T_{t}(x)}$ on $M_{T_{t}(x)}$. Under the condition of log-integrability of the cocycle $\left(F_{t}\right)$, that is, under the condition that

$$
\int_{X} \log \left\|F_{ \pm 1}\right\|_{x} d \mu(x)<+\infty
$$

the so-called Oseledets theorem states that there exists a collection of real numbers $\lambda_{1}>\cdots>\lambda_{k}, 1 \leq k \leq d$, such that, for $\mu$-almost every $x \in X$, one has a splitting

$$
M_{x}=E_{\lambda_{1}}^{\mu}(x) \oplus \cdots \oplus E_{\lambda_{k}}^{\mu}(x)
$$

with

$$
\lim _{t \rightarrow \pm \infty} \frac{1}{n} \log \left|F_{t}\left(v_{i}\right)\right|_{T_{t}(x)}=\lambda_{i}
$$

for every $v_{i} \in E_{\lambda_{i}}^{\mu}(x)-\{0\}$. Moreover, the subspaces $E_{\lambda_{i}}^{\mu}(x)$ depend measurably on $x \in X$. In the literature, the numbers $\lambda_{i}$ are called Lyapunov exponents and the subspaces $E_{\lambda_{i}}^{\mu}(x)$ are called Oseledets subspaces.

For the sake of convenience, one writes the list of Lyapunov exponents as $\lambda_{1} \geq \cdots \geq \lambda_{d}$ (where $d$ is the dimension of the fibers of the vector bundle) by repeating each exponent $\lambda_{i}$ a number of times equal to the real or complex dimension of the corresponding Oseledets space $\left.E_{\lambda_{i}}^{\mu}(x)\right) \subset M_{x}$ (which by ergodicity is constant almost everywhere). We will loosely refer to both the list $\lambda_{1}>\cdots>\lambda_{k}$ and $\lambda_{1} \geq \cdots \geq \lambda_{d}$ as the Lyapunov spectrum of the linear cocycle $\left(F_{t}\right)_{t \in \mathbb{R}}$, although the second list also contains the information about the real or complex multiplicities of Lyapunov exponents (that is, about the real or complex dimensions of the Oseledets subspaces).

The following general facts will be relevant in this paper:

- the natural complexification of any real linear cocycle has the same Lyapunov spectrum of the original real linear cocycle; in particular, the complexified cocycle has complex multiplicities equal to the real multiplicities of the original real cocycle;

- the Lyapunov spectrum of a real symplectic cocycle, that is, a real cocycle preserving a family of symplectic forms on the fibers $M_{x} \simeq$ $\mathbb{R}^{d}, d=2 n$, of the vector bundle $\pi: M \rightarrow X$, has the form

$$
\lambda_{1} \geq \cdots \geq \lambda_{n} \geq-\lambda_{n} \geq \cdots \geq-\lambda_{1} ;
$$

in other words, the Lyapunov spectrum of a symplectic cocycle is symmetric with respect to the origin $0 \in \mathbb{R}$. 
For more details on Oseledets theorem and the general theory of Lyapunov exponents, see the books [BDV] and [HK] (and references therein).

Coming back to the Kontsevich-Zorich cocycle, let us recall that, by Corollary 2.1, for any (Teichmüller) flow-invariant Borel probability ergodic measure $\mu$ on $\mathcal{H}_{g}^{(1)}$, the cocycle is log-integrable with respect to the Hodge norm, hence it has well-defined Lyapunov spectrum, with top exponent $\lambda_{1}=1$; since the cocycle on $H_{\mathbb{R}}^{1}$ preserves the symplectic intersection form on the ( $2 g$-dimensional) fibers $H^{1}(S, \mathbb{R})$ of $H_{\mathbb{R}}^{1}$, its Lyapunov spectrum is symmetric. Thus the Lyapunov spectrum of the Kontsevich-Zorich cocycle with respect to any (Teichmüller) flow-invariant Borel probability ergodic measure $\mu$ on $\mathcal{H}_{g}^{(1)}$ has the following form:

$$
\lambda_{1}^{\mu}=1 \geq \lambda_{2}^{\mu} \geq \cdots \geq \lambda_{g}^{\mu} \geq-\lambda_{g}^{\mu} \geq \cdots \geq-\lambda_{2}^{\mu} \geq-\lambda_{1}^{\mu}=-1 .
$$

In particular, the Kontsevich-Zorich spectrum always has $g$ non-negative and $g$ non-positive exponents. Let

$$
\lambda_{(1)}^{\mu}>\cdots>\lambda_{(n)}^{\mu}>-\lambda_{(n)}^{\mu}>\cdots>-\lambda_{(1)}^{\mu} .
$$

be the Kontsevich-Zorich spectrum of all distinct non-zero Lyapunov exponents of the Hodge bundle $H_{\mathbb{R}}^{1}$. Applying the Teichmüller flow both in forward and backward directions we get the corresponding Oseledets decomposition

$$
E_{\lambda_{(1)}^{\mu}}^{\mu} \oplus \cdots \oplus E_{\lambda_{(n)}^{\mu}}^{\mu} \oplus E_{(0)}^{\mu} \oplus E_{-\lambda_{(n)}^{\mu}}^{\mu} \oplus \cdots \oplus E_{-\lambda_{(1)}^{\mu}}^{\mu}
$$

at $\mu$-almost every point $(S, \omega)$ of $\mathcal{H}_{g}^{(1)}$, where $E_{(0)}^{\mu}$ is omitted if the Lyapunov spectrum of $\mu$ does not contain zero. By definition all nonzero vectors of each subspace $E_{\lambda_{(k)}^{\mu}}^{\mu}$ or $E_{(0)}^{\mu}$ share the same Lyapunov exponent $\lambda_{(k)}^{\mu}$ (correspondingly 0) which changes sign under the time reversing.

Remark 3.1. By convention, when saying that a measure (function, line subbundle, etc) is "invariant" we mean that it is "invariant with respect to the Teichmüller flow". If the corresponding object is "invariant with respect to the $\operatorname{SL}(2, \mathbb{R})$-action", we explicitly indicate that it is "SL $(2, \mathbb{R})$ invariant". In particular, decomposition (3.2) is defined by any probability measure invariant and ergodic with respect to the Teichmüller flow.

Lemma 3.1. Every subspace $E_{\lambda_{(i)}^{\mu}}^{\mu}$ of the Oseledets direct sum decomposition (3.2) except $E_{(0)}^{\mu}$ is isotropic. Any pair of subspaces $E_{\lambda_{(i)}^{\mu}}^{\mu}, E_{\lambda_{(j)}^{\mu}}^{\mu}$ such that $\lambda_{(j)}^{\mu} \neq-\lambda_{(i)}^{\mu}$ is symplectic orthogonal. The restriction of the symplectic form to each subspace $E_{\lambda_{(i)}^{\mu}}^{\mu} \oplus E_{-\lambda_{(i)}^{\mu}}^{\mu}$, where $i \neq 0$, and on $E_{(0)}^{\mu}$ is nondegenerate. 
Proof. The absolute value of the symplectic product of any two cocycles $c_{1}, c_{2}$ in $H^{1}(S, \mathbb{R})$ is uniformly bounded on any compact part $\mathcal{K}$ of $\mathcal{H}_{g}^{(1)}$ by the product of their Hodge norms,

$$
\left|\left\langle c_{1}, c_{2}\right\rangle\right| \leq \operatorname{const}(\mathcal{K}) \cdot\left\|c_{1}\right\|_{\omega} \cdot\left\|c_{2}\right\|_{\omega} \quad \text { for any } \omega \in \mathcal{K} .
$$

By ergodicity of the flow, it returns infinitely often to the compact part $\mathcal{K}$.

Consider a pair of cocycles $c_{i}, c_{j}$ such that $c_{i} \in E_{\lambda_{(i)}^{\mu}}, c_{j} \in E_{\lambda_{(j)}^{\mu}}$ and such that $\lambda_{(i)}^{\mu} \neq-\lambda_{(j)}^{\mu}$. By definition of $E_{\lambda_{(i)}^{\mu}}$, we have

$$
\left\|G_{t}^{K Z}\left(c_{1}\right)\right\|_{\omega} \cdot\left\|G_{t}^{K Z}\left(c_{2}\right)\right\|_{\omega} \sim \exp \left(\left(\lambda_{(i)}^{\mu}+\lambda_{(j)}^{\mu}\right) t\right)
$$

When $\lambda_{(i)}^{\mu}+\lambda_{(j)}^{\mu}<0$ the latter expression tends to zero when $t \rightarrow+\infty$; when $\lambda_{(i)}^{\mu}+\lambda_{(j)}^{\mu}>0$ the latter expression tends to zero when $t \rightarrow-\infty$. In both cases, we conclude that for a subsequence of positive or negative times $t_{k}$ (chosen when the trajectory visits the compact set $\mathcal{K}$ ) the symplectic product $\left\langle G_{t_{k}}^{K Z}\left(c_{1}\right), G_{t_{k}}^{K Z}\left(c_{2}\right)\right\rangle$ tends to zero. Since the symplectic product is preserved by the flow this implies that it is equal to zero, in particular $\left\langle c_{1}, c_{2}\right\rangle=0$. Thus, we have proved that every subspace $E_{\lambda_{(i)}^{\mu}}^{\mu}$ except $E_{(0)}^{\mu}$ is isotropic, and that any pair of subspaces $E_{\lambda_{(i)}^{\mu}}^{\mu}, E_{\lambda_{(j)}^{\mu}}^{\mu}$ such that $\lambda_{(j)}^{\mu} \neq$ $-\lambda_{(i)}^{\mu}$ is symplectic orthogonal. Hence, the cohomology space decomposes into a direct sum of pairwise symplectic-orthogonal subspaces $E_{(0)}^{\mu}, E_{\lambda_{(1)}^{\mu}}^{\mu} \oplus$ $E_{-\lambda_{(1)}^{\mu}}^{\mu}$, etc, where we couple all pairs $E_{\lambda_{(i)}^{\mu}}^{\mu}$ and $E_{-\lambda_{(i)}^{\mu}}^{\mu}$. Since the symplectic form is nondegenerate and the summands are symplectic-orthogonal, it is nondegenerate on any summand.

For any $k \in\{1, \ldots, g\}$ such that $\lambda_{k}^{\mu}>\lambda_{k+1}^{\mu} \geq 0$, let $i(k)$ be the index of the exponent $\lambda_{k}^{\mu}$ in the ordering without multiplicities (3.1), $\lambda_{(i(k))}^{\mu}=\lambda_{k}^{\mu}$. Let us define the $k$-th unstable Oseledets subbundle as

$$
E_{k}^{+}:=E_{\lambda_{(1)}^{\mu}}^{\mu} \oplus \cdots \oplus E_{\lambda_{(i k))}^{\mu}}^{\mu}
$$

3.2. Formulas for the Kontsevich-Zorich exponents. Let $V \subseteq H_{\mathbb{R}}^{1}$ be any $r$-dimensional flow-invariant measurable subbundle of the Hodge bundle, almost everywhere defined with respect to a flow-invariant ergodic probability measure $\mu$ on the moduli space $\mathcal{H}_{g}^{(1)}$. Let us denote

$$
\lambda_{1}^{V, \mu} \geq \cdots \geq \lambda_{r}^{V, \mu}
$$

the Lyapunov spectrum of the restriction of the Kontsevich-Zorich cocycle to the subbundle $V \subset H_{\mathbb{R}}^{1}$ with respect to the Teichmüller geodesic flow and the invariant measure $\mu$ on $\mathcal{H}_{g}^{(1)}$. Let us also denote

$$
\lambda_{(1)}^{V, \mu}>\cdots>\lambda_{(s)}^{V, \mu}
$$


the Lyapunov spectrum of all distinct Lyapunov exponents and let

$$
V=V_{1}^{\mu} \oplus \cdots \oplus V_{s}^{\mu}
$$

be the corresponding Oseledets decomposition. It follows from Oseledets theorem that the Lyapunov exponents on $V$ form a subset of the Lyapunov spectrum (3.1) of the cocycle on the Hodge bundle, that is,

$$
\left\{\lambda_{(1)}^{V, \mu}, \ldots, \lambda_{(s)}^{V, \mu}\right\} \subset\left\{\lambda_{(1)}^{\mu}, \ldots, \lambda_{(n)}^{\mu},-\lambda_{(1)}^{\mu}, \ldots,-\lambda_{(n)}^{\mu}\right\} \cup\{0\},
$$

and the Oseledets subspaces $V_{1}^{\mu}, \ldots, V_{s}^{\mu}$ are the non-trivial intersections of Oseledets spaces for the cocycle on the full Hodge bundle $H_{\mathbb{R}}^{1}$, as in (3.2), and the subbundle $V \subset H_{\mathbb{R}}^{1}$, that is,

$$
\left\{V_{1}^{\mu}, \ldots, V_{s}^{\mu}\right\}=\bigcup_{i=1}^{n}\left\{E_{\lambda_{(i)}^{\mu}}^{\mu} \cap V, E_{-\lambda_{(i)}^{\mu}}^{\mu} \cap V\right\} \cup\left\{E_{(0)}^{\mu} \cap V\right\} \backslash\{\{0\}\},
$$

where $E_{(0)}^{\mu}$ is omitted if the Lyapunov spectrum of $\mu$ does not contain zero.

For any $k \in\{1, \ldots, g\}$ denote by $G_{k}\left(H_{\mathbb{R}}^{1}\right)$ the total space of the Grassmannian bundle of isotropic $k$-dimensional subspaces of the real Hodge bundle $H_{\mathbb{R}}^{1}$. Let us denote by $\mathcal{N}_{k}(\mu)$ the space of all Borel probability measures on $G_{k}\left(H_{\mathbb{R}}^{1}\right)$ which project onto any probability measure, absolutely continuous with respect to the flow-invariant ergodic probability measure $\mu$ on $\mathcal{H}_{g}^{(1)}$ under the canonical projection.

The Kontsevich-Zorich cocycle on the Hodge bundle $H_{\mathbb{R}}^{1}$ preserves the symplectic form in the fibers. Hence, it defines a natural action on the Grassmannian bundle $G_{k}\left(H_{\mathbb{R}}^{1}\right)$. Since the subbundle $V$ is flow-invariant, the measurable Grassmannian subbundle $G_{k}(V)$ is also flow-invariant. Let $\mathcal{J}_{k}(\mu) \subset \mathcal{N}_{k}(\mu)$ be the subset of those measures in $\mathcal{N}_{k}(\mu)$ that are invariant with respect to the Kontsevich-Zorich cocycle $\left\{G_{t}^{K Z}\right\}$ on $G_{k}\left(H_{\mathbb{R}}^{1}\right)$. Note that all measures $\nu \in \mathcal{J}_{k}(\mu)$ project onto the flow-invariant ergodic probability measure $\mu$ on $\mathcal{H}_{g}^{(1)}$ under the canonical projection. It follows that the set $\mathcal{J}_{k}(\mu)$ is a compact subset of the set of all Borel probability measures on the locally compact space $G_{k}\left(H_{\mathbb{R}}^{1}\right)$ endowed with the weak-star topology.

Consider a subbundle $V$ of the Hodge bundle satisfying the properties stated at the beginning of section 3.2. Ergodicity of the measure and invariance of the intersection form with respect to the flow implies that the restrictions of the symplectic form to $\mu$-almost any fiber of $V$ have the same rank. We do not exclude the situation when the resulting form is degenerate. Denote by $2 p$ the rank of the restriction of the symplectic form to $V$; denote by $l$ the difference $l=r-p$ between the dimension of the fiber of $V$ and $p$. The restriction of the symplectic form to $\mu$-almost any fiber of $V$ is nondegenerate if and only if $l=p$, otherwise the form is degenerate and 
$p<l$. Note also that $l$ is the maximal dimension of an isotropic subspace in the fiber of $V$.

Let $G_{k}(V)$ denote the total space of the Grassmannian bundle of all $k$ dimensional isotropic subspaces contained in the fibers of $V$. By definition, for $\mu$-almost all $\omega \in \mathcal{H}_{g}^{(1)}$, the fiber $G_{k}(V)_{\omega}$ of the bundle $G_{k}(V)$ is equal to the space of all $k$-dimensional isotropic subspaces of $V_{\omega}$. Clearly, for each individual fiber, one has $G_{k}(V)_{\omega} \subseteq G_{k}\left(H_{\mathbb{R}}^{1}\right)_{\omega}$, so the Grassmanian bundle $G_{k}(V)$ is a measurable subbundle of $G_{k}\left(H_{\mathbb{R}}^{1}\right)$.

Let $\mathcal{N}_{k}^{V}(\mu) \subset \mathcal{N}_{k}(\mu)$ be the subset of all Borel probability measures on $G_{k}\left(H_{\mathbb{R}}^{1}\right)$ essentially supported on a subset of the measurable Grassmannian bundle $G_{k}(V) \subset G_{k}\left(H_{\mathbb{R}}^{1}\right)$. Note that there is no flow-invariance assumption in the definition of the sets $\mathcal{N}_{k}(\mu)$ and $\mathcal{N}_{k}^{V}(\mu)$. In fact, we will prove existence of flow-invariant probability measures essentially supported on the Grassmannian $G_{k}(V) \subset G_{k}\left(H_{\mathbb{R}}^{1}\right)$, which project to any given flow-invariant ergodic probability measure $\mu$ on $\mathcal{H}_{g}^{(1)}$, in Lemma 3.3 below.

We start with an elementary preparatory Lemma.

Lemma 3.2. Let $\bar{\mu}$ be any Borel probability measure absolutely continuous with respect to $\mu$ on $\mathcal{H}_{g}^{(1)}$. Let $V \subset H_{\mathbb{R}}^{1}$ be a measurable subbundle defined $\bar{\mu}$-almost everywhere. For any $k$ such that $G_{k}(V)$ is non-empty (i.e., for any $k \leq l)$, there exist measures on $G_{k}(V)$ which project onto $\bar{\mu}$ on $\mathcal{H}_{g}^{(1)}$, under the canonical projection. In particular, the space $\mathcal{N}_{k}^{V}(\mu)$ is also non-empty.

Proof. Any Borel measurable bundle can be trivialized on the complement of a subset of measure zero with respect to any given Borel measure. So, there exists a set $\mathcal{E}_{g} \subset \mathcal{H}_{g}^{(1)}$ of full $\bar{\mu}$-measure such that the restriction $\left.V\right|_{\mathcal{E}_{g}}$ is measurably isomorphic to the product bundle $\mathcal{E}_{g} \times \mathbb{R}^{r}$. It follows that the restriction $\left.G_{k}(V)\right|_{\mathcal{E}_{g}}$ of the Grassmannian bundle of isotropic subspaces is isomorphic to the product bundle $\mathcal{E}_{g} \times G_{k}\left(\mathbb{R}^{r}\right)$. By definition, for any Borel probability measure $\eta$ on $G_{k}\left(\mathbb{R}^{r}\right)$, the product measure $\bar{\mu} \times \eta$ on the space $\mathcal{E}_{g} \times G_{k}\left(\mathbb{R}^{r}\right)$ induces (by push-forward under the bundle isomorphism) a Borel probability measure $\nu$ on $G_{k}(V)$, which projects onto $\bar{\mu}$ on $\mathcal{H}_{g}^{(1)}$ under the canonical projection.

Note that we do not assume that the measure $\nu \in \mathcal{N}_{k}^{V}(\mu)$ constructed above is flow-invariant. Let $\mathcal{J}_{k}^{V}(\mu)=\mathcal{J}_{k}(\mu) \cap \mathcal{N}_{k}^{V}(\mu)$ be the subset of those measures in $\mathcal{N}_{k}^{V}(\mu)$ that are invariant with respect to the restriction of the Kontsevich-Zorich cocycle $\left\{G_{t}^{K Z}\right\}$ to $V$. We are going to show that the set $\mathrm{J}_{k}^{V}(\mu)$ is non-empty whenever the set $\mathcal{N}_{k}^{V}(\mu)$ is non-empty. 
For any $\nu \in \mathcal{N}_{k}(\mu)$, let $I(\nu) \subseteq \mathcal{N}_{k}(\mu)$ be the set of all weak limits of the family of probability measures

$$
\left\{\nu_{T}:=\frac{1}{T} \int_{0}^{T}\left(G_{t}^{K Z}\right)_{*}(\nu) d t \mid T>0\right\}
$$

in the space of all Borel probability measures on the locally compact space $G_{k}\left(H_{\mathbb{R}}^{1}\right)$. Here by a "weak limit" we mean any limit in the weak-star topology along some diverging sequence of positive times $T_{1}, T_{2}, \ldots, T_{n}, \ldots$ Of course, in general distinct sequences may lead to different weak limits.

Lemma 3.3. For any $\nu \in \mathcal{N}_{k}^{V}(\mu)$, the set $I(\nu)$ is a non-empty compact subset of the set $\mathcal{J}_{k}^{V}(\mu)$ of probability measures in $\mathcal{N}_{k}^{V}(\mu)$ which are invariant under the Kontsevich-Zorich cocycle.

Proof. The fiber of the Grassmannian bundle $G_{k}\left(H_{\mathbb{R}}^{1}\right)$ is the space $G_{k}\left(\mathbb{R}^{2 g}\right)$ of all isotropic subspaces of dimension $k$ in a symplectic space of dimension $2 g$, so that the fiber is a compact manifold. Also, the full Grassmannian bundle $G_{k}\left(H_{\mathbb{R}}^{1}\right)$ is a continuous bundle. Thus, the space of all Borel measures on $G_{k}\left(H_{\mathbb{R}}^{1}\right)$ of finite total mass is a Montel space (in the sense that all closed bounded sets are compact) with respect to the weak-star topology. Hence, for any diverging sequence of (positive) times $\left(T_{n}\right)$ we can extract from the sequence $\left\{\nu_{T_{n}}\right\}$ of measures in $\mathcal{N}_{k}(\mu)$ a converging subsequence. The limit measure is a probability measure since, by the Birkhoff ergodic theorem, the projection of the sequence $\left\{\nu_{T_{n}}\right\}$ under the canonical projection converges weakly to the flow-invariant ergodic probability measure $\mu$ on $\mathcal{H}_{g}^{(1)}$. It follows that the subset $I(\nu)$ of $\mathcal{J}_{k}(\mu)$ is nonempty. Since the set of all accumulation points of any given set in a topological space is closed and the set $\mathcal{J}_{k}(\mu)$ is compact, we get that $I(\nu)$ is a nonempty compact subset of $\mathcal{J}_{k}(\mu)$. As the flow $\left\{G_{t}^{K Z}\right\}$ is continuous on $G_{k}\left(H_{\mathbb{R}}^{1}\right)$, by the usual (relative) Bogolyubov-Krylov argument (see, e.g., page 135 of the book [HK]), one has that any measure $\hat{\nu} \in I(\nu)$ is $G_{t}^{K Z}$-invariant. We reproduce the argument below for the convenience of the reader. For any (fixed) $s \in \mathbb{R}$ and for all $T>0$, we have

$$
\begin{aligned}
\left(G_{s}^{K Z}\right)_{*}\left(\nu_{T}\right)-\nu_{T} & =\frac{1}{T}\left(\int_{0}^{T}\left(G_{t+s}^{K Z}\right)_{*}(\nu) d t-\int_{0}^{T}\left(G_{t}^{K Z}\right)_{*}(\nu) d t\right) \\
& =\frac{1}{T}\left(\int_{s}^{T+s}\left(G_{t}^{K Z}\right)_{*}(\nu) d t-\int_{0}^{T}\left(G_{t}^{K Z}\right)_{*}(\nu) d t\right) \\
& =\frac{1}{T}\left(\int_{T}^{T+s}\left(G_{t}^{K Z}\right)_{*}(\nu) d t-\int_{0}^{s}\left(G_{t}^{K Z}\right)_{*}(\nu) d t\right) .
\end{aligned}
$$


It follows that (for fixed $s \in \mathbb{R}$ ) the total mass $\left\|\left(G_{s}^{K Z}\right)_{*}\left(\nu_{T}\right)-\nu_{T}\right\|$ of the signed measure $\left(G_{s}^{K Z}\right)_{*}\left(\nu_{T}\right)-\nu_{T}$ converges to zero as $T \rightarrow \infty$. In fact,

$$
\left\|\left(G_{s}^{K Z}\right)_{*}\left(\nu_{T}\right)-\nu_{T}\right\| \leq \frac{2 s}{T} \rightarrow 0 .
$$

Let then $\left\{\nu_{T_{n}}\right\}$ be a sequence converging weakly to a measure $\hat{\nu} \in I(\nu)$. Since the map $G_{s}^{K Z}$ is continuous on $G_{k}\left(H_{\mathbb{R}}^{1}\right)$, the sequence $\left\{\left(G_{s}^{K Z}\right)_{*}\left(\nu_{T_{n}}\right)\right\}$ converges weakly to $\left(G_{s}^{K Z}\right)_{*}(\hat{\nu})$, hence, for all $s \in \mathbb{R}$, we have

$$
\left(G_{s}^{K Z}\right)_{*}(\hat{\nu})-\hat{\nu}=\lim _{n \rightarrow+\infty}\left(G_{s}^{K Z}\right)_{*}\left(\nu_{T_{n}}\right)-\nu_{T_{n}}=0 .
$$

We conclude that any measure $\hat{\nu} \in I(\nu)$ is $\left\{G_{t}^{K Z}\right\}$-invariant as stated.

It remains only to show that $I(\nu) \subset \mathcal{N}_{k}^{V}(\mu)$, that is, any measure $\hat{\nu} \in I(\nu)$ is essentially supported on $G_{k}(V)$, in the sense that $\hat{\nu}\left(G_{k}(V)\right)=1$.

Let $\bar{\mu}$ denote the projection of $\nu \in \mathcal{N}_{k}(\mu)$ on $\mathcal{H}_{g}$. By definition, $\bar{\mu}$ is absolutely continuous with respect to $\mu$ : in particular, given $\varepsilon>0$, we can choose $\delta(\varepsilon)>0$ such that $\mu(A)<\delta(\varepsilon)$ implies $\bar{\mu}(A)<\varepsilon$ for all measurable $A \subset \mathcal{H}_{g}$. On the other hand, by Luzin's theorem (see, e.g., page 2 of the book [M] $)$, given $\varepsilon>0$, we can fix $K_{\varepsilon} \subset \mathcal{H}_{g}^{(1)}$ a compact subset such that $\mu\left(K_{\varepsilon}\right)>1-\delta(\varepsilon)$ and $\left.V\right|_{K_{\varepsilon}}$ is a continuous subbundle of the measurable bundle $V$. In particular, $\left.G_{k}(V)\right|_{K_{\varepsilon}}$ is a compact subset of $G_{k}\left(H_{\mathbb{R}}^{1}\right)$. Let $\varphi$ be any real-valued continuous function on $G_{k}\left(H_{\mathbb{R}}^{1}\right)$ such that $0 \leq \varphi \leq 1$ and $\varphi$ is identically equal to 1 on $\left.G_{k}(V)\right|_{K_{\varepsilon}}$. Since, by definition, $\nu \in \mathcal{N}_{k}^{V}(\mu)$ means that $\nu$ is supported on $G_{k}(V)$ and it projects to $\bar{\mu} \ll \mu$ on $\mathcal{H}_{g}^{(1)}$, it follows from our choice of $\delta(\varepsilon)>0$ above that, for any $T>0$,

$$
\int_{G_{k}\left(H_{\mathbb{R}}^{1}\right)} \varphi d \nu_{T} \geq 1-\varepsilon .
$$

Hence, for any weak limit $\hat{\nu} \in I(\nu)$, one has

$$
\int_{G_{k}\left(H_{\mathbb{R}}^{1}\right)} \varphi d \hat{\nu} \geq 1-\varepsilon .
$$

Because this holds for every $\varphi$ as above, we conclude that

$$
\hat{\nu}\left(\left.G_{k}(V)\right|_{K_{\varepsilon}}\right) \geq 1-\varepsilon
$$

and hence $\hat{\nu}\left(G_{k}(V)\right)=1$, as claimed.

For any measure $\nu \in \mathcal{N}_{k}^{V}(\mu)$ we define below the average Lyapunov exponent $\Lambda^{(k)}(\nu)$ over the Grassmannian bundle $G_{k}(V)$. Let us consider an isotropic subspace $I$ in the fiber of the Hodge bundle over some point $\omega \in \mathcal{H}_{g}^{(1)}$. Let $\left\{c_{1}, \ldots, c_{k}\right\}$ and $\left\{c_{1}^{\prime}, \ldots, c_{k}^{\prime}\right\}$ be a pair of bases in it. Let us consider the parallel transport of these vectors to a neighborhood of $\omega$. Clearly, at any point of the neighborhood of $\omega$ the polyvectors $c_{1} \wedge \cdots \wedge c_{k}$ 
and $c_{1}^{\prime} \wedge \cdots \wedge c_{k}^{\prime}$ remain proportional with the same constant coefficient. Hence, the Hodge norms of these polyvectors are proportional with the same constant coefficient. This implies, in particular, that the logarithmic derivative of the Hodge norm of a polyvector along the Teichmüller geodesic flow, depends only on the isotropic subspace $I$,

$$
\mathcal{L} \log \left\|c_{1} \wedge \cdots \wedge c_{k}\right\|_{\omega}=\mathcal{L} \log \left\|c_{1}^{\prime} \wedge \cdots \wedge c_{k}^{\prime}\right\|_{\omega} .
$$

Thus, slightly abusing notations, in the following we shall sometimes denote the logarithmic derivative as above by

$$
\left.\frac{d \log \left\|G_{t}^{K Z}(\omega, I)\right\|}{d t}\right|_{t=0}:=\mathcal{L} \log \|I\|_{\omega}:=\mathcal{L} \log \left\|c_{1} \wedge \cdots \wedge c_{k}\right\|_{\omega} .
$$

The Oseledets theorem establishes that, for $\mu$-almost every Abelian differential $\omega \in \mathcal{H}_{g}^{(1)}$ on a Riemann surface $S$ and for every polyvector $c_{1} \wedge \cdots \wedge c_{k}$ in $\Lambda^{k}\left(H^{1}(S, \mathbb{R})\right)$, the Lyapunov exponent

$$
\lambda_{\omega}^{\mu}\left(c_{1}, \ldots, c_{k}\right):=\lim _{T \rightarrow+\infty} \frac{1}{T} \log \left\|G_{T}^{K Z}\left(c_{1} \wedge \cdots \wedge c_{k}\right)\right\|
$$

is well defined. Let us assume that the vectors $c_{1}, \ldots, c_{k}$ form a basis of an isotropic subspace $I_{k} \subset V_{\omega}$, and that the norm is the Hodge norm. Then, according to the above discussion about the logarithmic derivative of the Hodge norm of an isotropic subspace, for $\mu$-almost all $\omega \in \mathcal{H}_{g}^{(1)}$, the Lyapunov exponent $\lambda_{\omega}^{\mu}\left(c_{1}, \ldots, c_{k}\right)$ depends only on the isotropic subspace $I_{k} \subset V$ and can be written as follows:

$$
\begin{aligned}
& \lim _{T \rightarrow+\infty} \frac{1}{T} \log \left\|G_{T}^{K Z}\left(c_{1} \wedge \cdots \wedge c_{k}\right)\right\| \\
&=\lim _{T \rightarrow+\infty} \frac{1}{T} \int_{0}^{T} \frac{d}{d t} \log \left\|G_{t}^{K Z}\left(c_{1} \wedge \cdots \wedge c_{k}\right)\right\| d t \\
& \quad=\lim _{T \rightarrow+\infty} \frac{1}{T} \int_{0}^{T} \frac{d}{d t} \log \left\|G_{t}^{K Z}\left(\omega, I_{k}\right)\right\| d t .
\end{aligned}
$$

By Corollary 2.2 the function $\left|\frac{d}{d t} \log \left\|G_{t}^{K Z}\left(\omega, I_{k}\right)\right\|\right|$ is bounded above by $k \in \mathbb{N}$ for any point $\left(\omega, I_{k}\right)$ of the Grassmannian $G_{k}\left(H_{\mathbb{R}}^{1}\right)$. Hence, for any $\left(\omega, I_{k}\right) \in G_{k}\left(H_{\mathbb{R}}^{1}\right)$ and any $T>0$, we get the following uniform estimate:

$$
-k \leq \frac{1}{T} \int_{0}^{T} \frac{d}{d t} \log \left\|G_{t}^{K Z}\left(\omega, I_{k}\right)\right\| d t \leq k .
$$

By averaging the Lyapunov exponent $\lambda_{\omega}^{\mu}\left(c_{1}, \ldots, c_{k}\right)$ over $G_{k}(V)$ with respect to measure $\nu \in \mathcal{N}_{k}^{V}(\mu)$, we define the average Lyapunov exponent: 


$$
\begin{aligned}
\Lambda^{(k)}(\nu) & :=\int_{G_{k}(V)} \lim _{T \rightarrow+\infty}\left(\frac{1}{T} \int_{0}^{T} \frac{d}{d t} \log \left\|G_{t}^{K Z}\left(\omega, I_{k}\right)\right\| d t\right) d \nu \\
& =\lim _{T \rightarrow+\infty} \int_{G_{k}(V)}\left(\frac{1}{T} \int_{0}^{T} \frac{d}{d t} \log \left\|G_{t}^{K Z}\left(\omega, I_{k}\right)\right\| d t\right) d \nu \\
& =\lim _{T \rightarrow+\infty} \frac{1}{T} \int_{0}^{T} \int_{G_{k}(V)} \frac{d}{d t} \log \left\|G_{t}^{K Z}\left(\omega, I_{k}\right)\right\| d \nu d t .
\end{aligned}
$$

Note that the interchange of the limit with the integral and the change in the order of integration (Fubini theorem) in formula (3.7) are justified by the uniform upper bound established above in formula (3.6). Also, note that the definition of $\Lambda^{(k)}(\nu)$ doesn't assume the flow-invariance of $\nu \in \mathcal{N}_{k}^{V}(\mu)$.

Suppose now that $\mu$ is an ergodic $\operatorname{SL}(2, \mathbb{R})$-invariant probability measure on $\mathcal{H}_{g}^{(1)}$ (and not just flow-invariant as above). Let $V \subset H_{\mathbb{R}}^{1}$ be any $\mathrm{SO}(2, \mathbb{R})$-invariant measurable subbundle defined $\mu$-almost everywhere. A measure $\nu \in \mathcal{N}_{k}^{V}(\mu)$ will be called $\operatorname{SO}(2, \mathbb{R})$-invariant if it is invariant under the natural lift of the action of the group $\mathrm{SO}(2, \mathbb{R})$ to the Grassmannian bundle $G_{k}(V)$. The subset $\mathcal{O}_{k}^{V}(\mu) \subset \mathcal{N}_{k}^{V}(\mu)$ consisting of $\operatorname{SO}(2, \mathbb{R})$ invariant probability measures is non-empty whenever $\mathcal{N}_{k}^{V}(\mu)$ is. In fact, since by assumption the measure $\mu$ is $\mathrm{SL}(2, \mathbb{R})$-invariant and the bundle $V$ is $\mathrm{SO}(2, \mathbb{R})$-invariant, and since $\mathrm{SO}(2, \mathbb{R})$ is an amenable (compact) group, the $\mathrm{SO}(2, \mathbb{R})$-average of any probability measure in $\mathcal{N}_{k}^{V}(\mu)$ is a well-defined probability measure in $\mathcal{O}_{k}^{V}(\mu)$.

Theorem 1. Let $\mu$ be any $\mathrm{SL}(2, \mathbb{R})$-invariant Borel probability ergodic measure on the moduli space $\mathcal{H}_{g}^{(1)}$ of normalized Abelian differentials. Let $V \subset H_{\mathbb{R}}^{1}$ be any $\operatorname{SL}(2, \mathbb{R})$-invariant measurable subbundle defined $\mu$-almost everywhere. For any $\mathrm{SO}(2, \mathbb{R})$-invariant probability measure $\nu \in \mathcal{O}_{k}^{V}(\mu)$, the following formula holds:

$$
\Lambda^{(k)}(\nu)=\int_{G_{k}(V)} \Phi_{k}\left(\omega, I_{k}\right) d \hat{\nu}, \quad \text { for any } \hat{\nu} \in I(\nu) .
$$

Proof. Let $D$ denote the Poincaré disk and let $(t, \theta) \in D$ denote the geodesic polar coordinates on the Teichmüller disk $\mathrm{SO}(2, \mathbb{R}) \backslash \mathrm{SL}(2, \mathbb{R}) \cdot \omega$ centered at an Abelian differential $\omega \in \mathcal{H}_{g}^{(1)}$ on a Riemann surface $S$, defined as follows. Let $\mathrm{SO}(2, \mathbb{R}):=\left\{R_{\theta} \mid \theta \in[0,2 \pi)\right\}$ and let

$$
\left(S_{(t, \theta)}, \omega_{(t, \theta)}\right):=\left(G_{t} \circ R_{\theta}\right)(S, \omega), \quad \text { for all }(t, \theta) \in \mathbb{R}^{+} \times[0,2 \pi) .
$$

For $\mu$-almost all Abelian differential $\omega \in \mathcal{H}_{g}^{(1)}$ on a Riemann surface $S$ and for any $k$-dimensional isotropic subspace $I_{k} \subset V_{\omega}$, let $\left\{c_{1}, \ldots, c_{k}\right\} \subset I_{k}$ be any Hodge orthonormal basis at $(S, \omega)$ and let $\left\|c_{1} \wedge \cdots \wedge c_{k}\right\|_{(\omega, t, \theta)}$ denote 
the Hodge norm of the polyvector $c_{1} \wedge \cdots \wedge c_{k} \in \Lambda^{k}\left(H^{1}(S, \mathbb{R})\right)$ at the point $\left(S_{(t, \theta)}, \omega_{(t, \theta)}\right) \in \mathrm{SO}(2, \mathbb{R}) \backslash \mathrm{SL}(2, \mathbb{R}) \cdot \omega$ of coordinates $(t, \theta) \in D$.

From the variational formulas of Lemma 2.9 for the hyperbolic Laplacian of the Hodge norm of a polyvector on a Teichmüller disk, by the Green formula (or, equivalently, by explicit integration of the Poisson equation for the hyperbolic Laplacian on the Poincaré disk $D$ ), we derive the formula stated below (see formula (5.10) in $[\overline{\mathrm{F} 2}]$ ). Let $D_{t}$ denote the disk of hyperbolic radius $t>0$ centered at the origin of the Poincaré disk, let $\left|D_{t}\right|$ denote its hyperbolic area and let $\mathcal{A}_{P}$ denote the Poincaré area form. Let us also adopt the convention, introduced above, on the logarithmic derivative of the Hodge norm of isotropic subspaces. We have

$$
\begin{gathered}
\frac{1}{2 \pi} \int_{0}^{2 \pi} \frac{\partial}{\partial t} \log \left\|\left(G_{t}^{K Z} \circ R_{\theta}\right)\left(\omega, I_{k}\right)\right\| d \theta \\
=\frac{1}{2 \pi} \int_{0}^{2 \pi} \frac{\partial}{\partial t} \log \left\|c_{1} \wedge \cdots \wedge c_{k}\right\|_{(\omega, t, \theta)} d \theta \\
=\frac{\tanh t}{\left|D_{t}\right|} \int_{D_{t}}\left(\Phi_{k} \circ G_{\tau}^{K Z} \circ R_{\theta}\right)\left(\omega, I_{k}\right) d \mathcal{A}_{P}(\tau, \theta)
\end{gathered}
$$

Let us now integrate formula (3.9) over the Grassmannian $G_{k}(V)$ with respect to the $\mathrm{SO}(2, \mathbb{R})$-invariant probability measure $\nu \in \mathcal{O}_{k}^{V}(\mu)$. Note that by Corollary 2.2 all the integrands are uniformly bounded, hence it is possible to exchange the order of integrations.

On the left-hand side (LHS for short) of formula (3.9), by the $\operatorname{SO}(2, \mathbb{R})$ invariance of the measure $\nu$ on $G_{k}(V)$, we compute as follows:

$$
\begin{aligned}
& \int_{G_{k}(V)} \frac{1}{2 \pi} \int_{0}^{2 \pi} \frac{\partial}{\partial t} \log \left\|\left(G_{t}^{K Z} \circ R_{\theta}\right)\left(\omega, I_{k}\right)\right\| d \theta d \nu \\
& =\frac{1}{2 \pi} \int_{0}^{2 \pi} \int_{G_{k}(V)} \frac{\partial}{\partial t} \log \left\|\left(G_{t}^{K Z} \circ R_{\theta}\right)\left(\omega, I_{k}\right)\right\| d \nu d \theta \\
& =\frac{1}{2 \pi} \int_{0}^{2 \pi} \int_{G_{k}(V)} \frac{d}{d t} \log \left\|G_{t}^{K Z}\left(\omega, I_{k}\right)\right\| d \nu d \theta \\
& =\int_{G_{k}(V)} \frac{d}{d t} \log \left\|G_{t}^{K Z}\left(\omega, I_{k}\right)\right\| d \nu .
\end{aligned}
$$

On the right-hand side (RHS for short) of formula (3.9) we compute as follows. Let us recall that the Poincaré area form can be written as $d \mathcal{A}_{P}(\tau, \theta)=$ $d\left(\sinh ^{2} \tau\right) d \theta$ in geodesic polar coordinates $(\tau, \theta) \in \mathbb{R}^{+} \times[0,2 \pi)$ and, as a consequence, the Poincaré area of the disk $D_{t}$ of geodesic radius $t>0$ is $\left|D_{t}\right|=2 \pi \sinh ^{2} t$. 
By taking into account the uniform bound (2.48) for $\left|\Phi_{k}\left(\omega, I_{k}\right)\right|$, and by the above elementary formulas of hyperbolic geometry, and the $\operatorname{SO}(2, \mathbb{R})$ invariance of the measure $\nu$ on $G_{k}(V)$, we proceed as follows:

$$
\begin{aligned}
& \frac{1}{2 \pi} \int_{G_{k}(V)} \int_{D_{t}}\left(\Phi_{k} \circ G_{\tau}^{K Z} \circ R_{\theta}\right)\left(\omega, I_{k}\right) d \mathcal{A}_{P}(\tau, \theta) d \nu \\
& =\frac{1}{2 \pi} \int_{0}^{2 \pi} \int_{G_{k}(V)} \int_{0}^{t}\left(\Phi_{k} \circ G_{\tau}^{K Z} \circ R_{\theta}\right)\left(\omega, I_{k}\right) d\left(\sinh ^{2} \tau\right) d \nu d \theta \\
& =\int_{G_{k}(V)} \int_{0}^{t}\left(\Phi_{k} \circ G_{\tau}^{K Z}\right)\left(\omega, I_{k}\right) d\left(\sinh ^{2} \tau\right) d \nu .
\end{aligned}
$$

To sum up our computations so far, by integration of formula (3.9) over the Grassmannian $G_{k}(V)$ with respect to the $\mathrm{SO}(2, \mathbb{R})$-invariant probability measure $\nu \in \mathcal{O}_{k}^{V}(\mu)$ we have:

$$
\begin{aligned}
\int_{G_{k}(V)} \frac{d}{d t} \log \left\|G_{t}^{K Z}\left(\omega, I_{k}\right)\right\| d \nu \\
=\frac{\tanh t}{\sinh ^{2} t} \int_{G_{k}(V)} \int_{0}^{t} \Phi_{k} \circ G_{\tau}^{K Z} d\left(\sinh ^{2} \tau\right) d \nu .
\end{aligned}
$$

Let us then average the above formula over the interval $[0, T] \subset \mathbb{R}$ and take the limit as $T \rightarrow+\infty$. The average of the LHS of formula (3.12) converges, by the definition in formula (3.7), to the average Lyapunov exponent of the $\mathrm{SO}(2, \mathbb{R})$-invariant measure $\nu \in \mathcal{O}_{k}^{V}(\mu)$, that is,

$$
\Lambda^{(k)}(\nu)=\lim _{T \rightarrow+\infty} \frac{1}{T} \int_{0}^{T} \int_{G_{k}(V)} \frac{d}{d t} \log \left\|G_{t}^{K Z}\left(\omega, I_{k}\right)\right\| d \nu d t .
$$

We claim that for any probability measure $\hat{\nu} \in I(\nu)$ there exists a diverging sequence $\left\{T_{n}\right\}$ such that the average over $\left[0, T_{n}\right]$ of the RHS of formula (3.12) converges to the integral

$$
\int_{G_{k}(V)} \Phi_{k}\left(\omega, I_{k}\right) d \hat{\nu}
$$

so that, taking into account the limit in formula (3.13), the theorem follows from formula (3.12). The above claim is proved as follows. For any continuous function $\varphi$ with compact support on $G_{k}\left(H_{\mathbb{R}}^{1}\right)$, the function

$$
\frac{\tanh t}{\sinh ^{2} t} \int_{0}^{t} \varphi \circ G_{\tau}^{K Z} d\left(\sinh ^{2} \tau\right)-\varphi \circ G_{t}^{K Z}
$$

converges to zero uniformly as $t \rightarrow+\infty$. In fact, the hyperbolic tangent converges to 1 , the function $\varphi$ is uniformly continuous, and for any $\varepsilon>0$, 
the mass assigned by the probability measure $d\left(\sinh ^{2} \tau\right) / \sinh ^{2} t$ over $[0, t]$ to the interval $[0, t-\varepsilon]$ converges to zero as $t \rightarrow+\infty$.

It follows that the measure

$$
\frac{1}{T} \int_{0}^{T} \frac{\tanh t}{\sinh ^{2} t} \int_{0}^{t}\left(G_{\tau}^{K Z}\right)_{*}(\nu) d\left(\sinh ^{2} \tau\right)-\frac{1}{T} \int_{0}^{T}\left(G_{t}^{K Z}\right)_{*}(\nu)
$$

converges to zero weakly as $T \rightarrow+\infty$. Thus for any $\hat{\nu} \in I(\nu)$ there exists a diverging sequence $\left\{T_{n}\right\}$ such that the sequence of measures

$$
\frac{1}{T_{n}} \int_{0}^{T_{n}} \frac{\tanh t}{\sinh ^{2} t} \int_{0}^{t}\left(G_{\tau}^{K Z}\right)_{*}(\nu) d\left(\sinh ^{2} \tau\right)
$$

converges weakly to the measure $\hat{\nu}$ on $G_{k}\left(H_{\mathbb{R}}^{1}\right)$, essentially supported on $G_{k}(V) \subset G_{k}\left(H_{\mathbb{R}}^{1}\right)$. Since the function $\Phi_{k}$ is continuous and bounded on $G_{k}\left(H_{\mathbb{R}}^{1}\right)$ it follows that the average over $\left[0, T_{n}\right]$ of the RHS of formula (3.12) converges to the integral in formula (3.14), as claimed, and the proof of the theorem is completed.

For any $k \in\{1, \ldots, r-1\}$ such that $\lambda_{k}^{V, \mu}>\lambda_{k+1}^{V, \mu}$, let $j(k)$ be the index such that $\lambda_{(j(k))}^{V, \mu}=\lambda_{k}^{V, \mu}$. Let us define

$$
V_{k}^{+}:=V_{1}^{\mu} \oplus \cdots \oplus V_{j(k)}^{\mu} .
$$

In general, the subbundle $V_{k}^{+}$does not need to be a bundle of isotropic subspaces, that is, a measurable section of the Grassmannian $G_{k}(V)$. However, note that if $\lambda_{k+1}^{V, \mu} \geq 0$, then the bundle $V_{k}^{+}$is a subbundle of the unstable Oseledets bundle which is isotropic (since the Kontsevich-Zorich cocycle is symplectic), hence it is itself isotropic.

Corollary 3.1. Let $\mu$ be any $\mathrm{SL}(2, \mathbb{R})$-invariant Borel probability ergodic measure on the moduli space $\mathcal{H}_{g}^{(1)}$ of normalized Abelian differentials. Let $V \subset H_{\mathbb{R}}^{1}$ be any $\mathrm{SL}(2, \mathbb{R})$-invariant measurable subbundle defined $\mu$-almost everywhere. Assume that there exists $k \in\{1, \ldots, \operatorname{dim}(V)-1\}$ such that $\lambda_{k}^{V, \mu}>\lambda_{k+1}^{V, \mu}$ and that the subbundle $V_{k}^{+}$is a bundle of $k$-dimensional isotropic subspaces (that is, it defines a measurable section of the Grassmanian $G_{k}(V)$ ). Then the following formula holds:

$$
\lambda_{1}^{V, \mu}+\cdots+\lambda_{k}^{V, \mu}=\int_{\mathcal{H}_{g}^{(1)}} \Phi_{k}\left(\omega, V_{k}^{+}(\omega)\right) d \mu(\omega) .
$$

Proof. Let $\nu_{k} \in \mathcal{O}_{k}^{V}(\mu)$ be an $\mathrm{SO}(2, \mathbb{R})$-invariant probability measure on $G_{k}(V)$ such that all of its conditional measures on the fibers $G_{k}(V)_{\omega}$ of the Grassmannian bundle are equivalent to the Lebesgue measure, for $\mu$-almost all $\omega \in \mathcal{H}_{g}^{(1)}$. Theorem 1 in this special case implies formula (3.15). In fact, by the assumption that $\lambda_{k}^{V, \mu}>\lambda_{k+1}^{V, \mu}$ the family of measures given in formula (3.5) converges to the unique probability measure $\hat{\nu}_{k}$ on $G_{k}(V)$ given 
by the condition that for $\mu$-almost all $\omega \in \mathcal{H}_{g}^{(1)}$ the conditional measure $\nu_{k} \mid G(V)_{\omega}$ is the Dirac measure at the point $V_{k}^{+}(\omega)$ (in other terms, the measure $\nu_{k}$ is defined as the push-forward of the measure $\mu$ on $\mathcal{H}_{g}^{(1)}$ under the section $\left.V_{k}^{+}: \mathcal{H}_{g}^{(1)} \rightarrow G_{k}(V)\right)$. In other words, the set $I\left(\nu_{k}\right)$ of all weak limits of the family of measures given in formula (3.5) is equal to $\left\{\hat{\nu}_{k}\right\}$. By the Oseledets theorem, the average Lyapunov exponent $\Lambda^{(k)}\left(\nu_{k}\right)$ of the Kontsevich-Zorich cocycle with respect to the measure $\nu_{k}$ on the bundle $G_{k}(V)$ is given by the formula

$$
\Lambda^{(k)}\left(\nu_{k}\right)=\lambda_{1}^{V, \mu}+\cdots+\lambda_{k}^{V, \mu} .
$$

Thus formula (3.15) is indeed a particular case of formula (3.8) .

In the particular case of the full Hodge bundle we derive below a result first proved in [ [F2], Corollary 5.5, for the canonical absolutely continuous invariant measures on connected components of strata of the moduli space.

Corollary 3.2. Let $\mu$ be any $\mathrm{SL}(2, \mathbb{R})$-invariant Borel probability ergodic measure on the moduli space $\mathcal{H}_{g}^{(1)}$ of normalized Abelian differentials. Assume that there exists $k \in\{1, \ldots, g-1\}$ such that $\lambda_{k}^{\mu}>\lambda_{k+1}^{\mu} \geq 0$. Then the following formula holds:

$$
\lambda_{1}^{\mu}+\cdots+\lambda_{k}^{\mu}=\int_{\mathcal{H}_{g}^{(1)}} \Phi_{k}\left(\omega, E_{k}^{+}(\omega)\right) d \mu(\omega) .
$$

By Remark 2.4 Corollary 3.2 for $k=g$ holds without any assumptions on the Lyapunov exponents and provides a version of the Kontsevich formula for their sum (see $[\underline{K}]$ and $[\bar{F} 2]$, Corollary 5.3):

Corollary 3.3. Let $\mu$ be any $\mathrm{SL}(2, \mathbb{R})$-invariant Borel probability ergodic measure on the moduli space $\mathcal{H}_{g}^{(1)}$ of normalized Abelian differentials. The following formula holds:

$$
\lambda_{1}^{\mu}+\cdots+\lambda_{g}^{\mu}=\int_{\mathcal{H}_{g}^{(1)}}\left(\Lambda_{1}+\cdots+\Lambda_{g}\right) d \mu .
$$

3.3. Reducibility of the second fundamental form. Let $V \subset H^{1}(S, \mathbb{R})$ be a subspace invariant under the Hodge operator. Since for any nonzero $c \in V$ one has $\|c\|=\langle c, * c\rangle>0$, this implies that $c$ cannot be symplecticorthogonal to $V$. Thus, invariance of $V$ under the Hodge star-operator implies, in particular, that restriction of the symplectic form to $V$ is nondegenerate, in particular, $V$ is even-dimensional. For any Hodge star-invariant subspace $V \subseteq H^{1}(S, \mathbb{R})$, let us define $V^{1,0} \subset H^{1,0}(S)$ and $V^{0,1} \subset H^{0,1}(S)$ to be the subspaces of cohomology classes of all holomorphic, respectively, anti-holomorphic forms $\omega$ such that $[\operatorname{Re}(\omega)] \in V$. Invariance of $V$ under the Hodge operator implies that the sets $V^{1,0}$ and $V^{0,1}$ are indeed complex 
vector spaces, that $V_{\mathbb{C}}=V^{1,0} \oplus V^{0,1}$ and that $\overline{V^{1,0}}=V^{0,1}$. In particular, $\operatorname{dim}_{\mathbb{R}} V=2 \operatorname{dim}_{\mathbb{C}} V^{1,0}$. Let us denote by $\left.H_{\omega}\right|_{V^{1,0}}$ and $\left.B_{\omega}\right|_{V^{1,0}}$ the restrictions of the forms $H_{\omega}$ and $B_{\omega}$ to $V^{1,0} \subseteq H^{1,0}(S)$ and by $\left.H_{\omega}^{\mathbb{R}}\right|_{V}$ and $\left.B_{\omega}^{\mathbb{R}}\right|_{V}$ the restrictions of the forms $H_{\omega}^{\mathbb{R}}$ and $B_{\omega}^{\mathbb{R}}$ to $V \subseteq H^{1}(S, \mathbb{R})$ respectively.

Lemma 3.4. A subspace $V \subset H^{1}(S, \mathbb{R})$ is invariant under the Hodge staroperator if and only if the subspace $V^{\perp}$, Hodge-orthogonal to $V$, coincides with the subspace $V^{\dagger}$, symplectic-orthogonal to $V$. In that case the subspace $V^{\perp}=V^{\dagger}$ is Hodge star-invariant and $\left(V^{1,0}\right)^{\perp}=\left(V^{\perp}\right)^{1,0}$.

Proof. Assume that a subspace $V \subset H^{1}(S, \mathbb{R})$ is Hodge star-invariant. Let $V^{\dagger}$ be the subspace symplectic-orthogonal to $V$. By (2.5) for any $c_{1} \in V^{\dagger}$ and $c_{2} \in V$ one has $\left(c_{1}, c_{2}\right)=\left\langle c_{1}, * c_{2}\right\rangle$. Since $V$ is Hodge star-invariant, we have $* c_{2} \in V$ and hence the right expression is equal to zero. It follows that $V^{\dagger} \subset V^{\perp}$. The converse inclusion is proved similarly. In fact, for any $c_{1} \in V^{\perp}$ and $c_{2} \in V$ one has $\left\langle c_{1}, c_{2}\right\rangle=-\left(c_{1}, * c_{2}\right)$. Again since $V$ is Hodge star-invariant, we have $* c_{2} \in V$, hence the right expression is equal to zero. Thus $V^{\perp} \subset V^{\dagger}$ and equality holds. Conversely, assume that $V^{\perp}=V^{\dagger}$. Let $c_{1} \in V$ and let $c_{2} \in V^{\perp}$. By (2.5), one has $\left(* c_{1}, c_{2}\right)=\left\langle c_{1}, c_{2}\right\rangle=0$, hence $* c_{1} \in\left(V^{\perp}\right)^{\perp}=V$. Thus $V$ is Hodge star-invariant.

Let us show that if $V$ is Hodge star-invariant, then $V^{\perp}$ is also Hodge starinvariant. Let $c_{2} \in V^{\perp}$ and take any $c_{1} \in V$. By the same equation (2.5) one has $\left\langle c_{1}, * c_{2}\right\rangle=\left(c_{1}, c_{2}\right)$. Since $c_{1}$ and $c_{2}$ belong to Hodge-orthogonal subspaces the right expression is equal to zero. Hence $* c_{2}$ is symplecticorthogonal to any $v$ in $V$, which implies that $* c_{2} \in V^{\perp}$.

Finally, for any $\omega_{1} \in V^{1,0}$ and any $\omega_{2} \in\left(V^{\perp}\right)^{1,0}$ let $c_{1}=\left[\operatorname{Re}\left(\omega_{1}\right)\right] \in V$ and let $c_{2}=\left[\operatorname{Re}\left(\omega_{2}\right)\right] \in V^{\perp}$. By formula (2.6)

$$
\left(\omega_{1}, \omega_{2}\right):=\left(h\left(c_{1}\right), h\left(c_{2}\right)\right)=\left(c_{1}, c_{2}\right)+i\left\langle c_{1}, c_{2}\right\rangle,
$$

which is equal to zero since $V$ and $V^{\perp}$ are both symplectic-orthogonal and Hodge-orthogonal. This implies that $\left(V^{1,0}\right)^{\perp}=\left(V^{\perp}\right)^{1,0}$.

Remark. Note that the above property is not related to either the $\operatorname{SL}(2, \mathbb{R})$ action or the Teichmüller flow.

Proposition 1. Let $V \subset H^{1}\left(S_{0}, \mathbb{R}\right)$ be a Hodge star-invariant subspace in the fiber of the Hodge bundle over $\left(S_{0}, \omega_{0}\right) \in \mathcal{H}_{g}^{(1)}$ and let $V^{\dagger} \subset H^{1}\left(S_{0}, \mathbb{R}\right)$ denote its symplectic orthogonal. Let $U=]-\varepsilon, \varepsilon[$ be any open interval along the trajectory of the Teichmüller flow passing through $\omega_{0}$. Let us identify the fibers of the Hodge bundle over $U$ by parallel transport with respect to the Gauss-Manin connection. The following properties are equivalent:

(i) For any $t \in U$ the subspace $V$ stays Hodge star-invariant at $\left(S_{t}, \omega_{t}\right)$.

(ii) For any $t \in U$ the subspaces $V$ and $V^{\dagger}$ are $B_{\omega_{t}}^{\mathbb{R}}$-orthogonal. 
An analogous equivalence holds when $U$ is replaced by a small open ball in $\mathrm{SL}(2, \mathbb{R})$ containing the identity element, or by a small open neighborhood of the initial point $\left(S_{0}, \omega_{0}\right)$ in its Teichmüller disc.

Proof. Suppose that property (i) is satisfied. By Lemma 3.4 we have a direct sum decomposition $H^{1}\left(S_{t}, \mathbb{R}\right)=V \oplus V^{\dagger}$ where $V$ and $V^{\dagger}$ are simultaneously symplectic-orthogonal and Hodge-orthogonal with respect to the Hodge-inner product $(\cdot, \cdot)_{\omega_{t}}$ on $H^{1}\left(S_{t}, \mathbb{R}\right)$. Since the symplectic structure is preserved by the Gauss-Manin connection, $V^{\dagger}$ is constant over $U$ under our identification of the real cohomology spaces $H^{1}\left(S_{t}, \mathbb{R}\right)$ given by the connection. Hence for any pair $\left(v, v^{\dagger}\right) \in V \times V^{\dagger}$, the Hodge inner products $\left(v, v^{\dagger}\right)_{\omega_{t}}=\left(* v, v^{\dagger}\right)_{\omega_{t}}=0$ for all $t \in U$, so that by Lemma 2.6

$$
\begin{aligned}
\frac{d}{d t}\left(v, v^{\dagger}\right)_{\omega_{t}} & =-2 \operatorname{Re} B_{\omega_{t}}^{\mathbb{R}}\left(v, v^{\dagger}\right)=0 \quad \text { and } \\
\frac{d}{d t}\left(* v, v^{\dagger}\right)_{\omega_{t}} & =-2 \operatorname{Re} B_{\omega_{t}}^{\mathbb{R}}\left(* v, v^{\dagger}\right)=2 \operatorname{Im} B_{\omega_{t}}^{\mathbb{R}}\left(v, v^{\dagger}\right)=0 .
\end{aligned}
$$

It follows that $V$ and $V^{\perp}$ are $B_{\omega_{t}}^{\mathbb{R}}$-orthogonal for all $t \in U$.

Conversely, suppose that property (ii) is satisfied. Let $V^{\dagger}$ be the symplectic orthogonal of $V$. Since the Gauss-Manin connection preserves the symplectic structure, the space $V^{\dagger}$ is constant over $U$. In addition, since $V$ is Hodge star-invariant, the symplectic-orthogonal $V^{\dagger}$ and the Hodgeorthogonal $V^{\perp}$ coincide at $t=0$. It follows from formulas (3.18) that since $V$ and $V^{\dagger}$ are $B_{\omega_{t}}^{\mathbb{R}}$-orthogonal for all $t \in U$ and they are Hodge-orthogonal for $t=0$, then they are Hodge-orthogonal for all $t \in U$. Thus the symplectic orthogonal and the Hodge-orthogonal subspaces of $V$ coincide, hence by Lemma 3.4 the space $V$ is Hodge star-invariant, for all $t \in U$.

In the setting of Proposition 1 let

$$
\Lambda_{1}^{V}\left(\omega_{t}\right) \geq \cdots \geq \Lambda_{n}^{V}\left(\omega_{t}\right) \geq 0
$$

be the eigenvalues of the positive-semidefinite Hermitian form $\left.H_{\omega_{t}}\right|_{V_{t}^{1,0}}$ restricted to $V_{t}^{1,0}$, where $n=\operatorname{dim}_{\mathbb{C}} V_{t}^{1,0}$.

Corollary 3.4. In the setting of Proposition 1 the following sets with multiplicities coincide:

$$
\left\{\Lambda_{1}(\omega), \ldots, \Lambda_{g}(\omega)\right\}=\left\{\Lambda_{1}^{V}(\omega), \ldots, \Lambda_{n}^{V}(\omega)\right\} \sqcup\left\{\Lambda_{1}^{V^{\perp}}(\omega), \ldots, \Lambda_{g-n}^{V^{\perp}}(\omega)\right\}
$$

Proof. Let $\left\{\omega_{1}, \ldots, \omega_{g}\right\}$ be an orthonormal basis such that $\left\{\omega_{1}, \ldots, \omega_{n}\right\}$ spans $V,\left\{\omega_{n+1}, \ldots, \omega_{g}\right\}$ spans $V^{\perp}$ and $B_{\omega}$ has block-diagonal matrix in the basis $\left\{\omega_{1}, \ldots, \omega_{g}\right\}$. By formula (2.31) the matrix $H_{\omega_{t}}$ is also blockdiagonal in the basis $\omega_{1}, \ldots, \omega_{g}$. Hence,

$$
H_{\omega_{t}}=\left.H_{\omega_{t}}\right|_{V_{t}^{1,0}}+\left.H_{\omega_{t}}\right|_{\left(V_{t}^{\perp}\right)^{1,0}}
$$


which is exactly the statement of the Corollary.

Remark. Suppose that at some point $(S, \omega)$ of the moduli space of normalized Abelian differentials $\mathcal{H}_{g}^{(1)}$ all the eigenvalues $\Lambda_{1}(\omega), \ldots, \Lambda_{g}(\omega)$ are distinct. The corollary above implies that there is only a finite number of subspaces (namely $2^{g}$ ) which might a priori serve as fibers of Hodge starinvariant subbundles, namely, those spanned by $\operatorname{Re}\left(\omega_{i_{j}}\right), \operatorname{Im}\left(\omega_{i_{j}}\right)$ for some subcollection $\left\{\omega_{i_{1}}, \ldots, \omega_{i_{k}}\right\}$ of eigenvectors $\left\{\omega_{1}, \ldots, \omega_{g}\right\}$ of $H_{\omega}$.

The condition that $B_{\omega}$ is block-diagonal in the corresponding basis in a small neighborhood $U$ of the initial point is a necessary and sufficient condition for extension of the corresponding subspace to a local Hodge starinvariant subbundle over $U$.

Let $V \subset H_{\mathbb{R}}^{1}$ be an $\operatorname{SL}(2, \mathbb{R})$-invariant and Hodge star-invariant subbundle of dimension $2 n$ over a full measure set for an $\operatorname{SL}(2, \mathbb{R})$-invariant ergodic probability measure $\mu$ on the moduli space $\mathcal{H}_{g}^{(1)}$ of normalized Abelian differentials. The Hodge star-invariance is a very strong property of an $\mathrm{SL}(2, \mathbb{R})$-invariant subbundle. In particular, the restriction of the Kontsevich-Zorich cocycle to $V$ mimics most of the properties of the cocycle on Hodge bundle $H_{\mathbb{R}}^{1}$, where $n$ plays a role of a "virtual genus". Let us give several illustrations of this general philosophy.

We have seen that the symplectic structure on $H_{\mathbb{R}}^{1}$ restricts to a nondegenerate symplectic structure on $V$, hence the Kontsevich-Zorich cocycle on $V$ is symplectic. It follows that for any $\mathrm{SL}(2, \mathbb{R})$-invariant Borel probability ergodic measure $\mu$ on $\mathcal{H}_{g}^{(1)}$ the Kontsevich-Zorich spectrum on $V$ is symmetric:

$$
\lambda_{1}^{V, \mu} \geq \cdots \geq \lambda_{n}^{\mu} \geq-\lambda_{n}^{V, \mu} \geq \cdots \geq-\lambda_{1}^{V, \mu} .
$$

We get the following generalization of the Kontsevich formula for the sum of positive Lyapunov exponents (compare analogous formulas in $[\mathrm{K}]$, [EKZ1] and, for the specific case of Teichmüller curves, in [BMo]).

Corollary 3.5. Let $\mu$ be any $\mathrm{SL}(2, \mathbb{R})$-invariant ergodic Borel probability measure on the moduli space $\mathcal{H}_{g}^{(1)}$. The following formula holds for the Kontsevich-Zorich exponents of any subbundle $V \subset H_{\mathbb{R}}^{1}$ (of dimension $2 n$ ), $\mu$-almost everywhere $\mathrm{SL}(2, \mathbb{R})$-invariant and Hodge star-invariant:

$$
\lambda_{1}^{V, \mu}+\cdots+\lambda_{n}^{V, \mu}=\int_{\mathcal{H}_{g}^{(1)}}\left(\Lambda_{1}^{V}+\cdots+\Lambda_{n}^{V}\right) d \mu .
$$

Proof. Let us consider a maximal isotropic subspace $I_{n}$ of $V_{\omega}$, and some Hodge-orthonormal basis $\left\{c_{1}, \ldots, c_{n}\right\}$ of $I_{n}$. Let $\left\{\omega_{1}, \ldots, \omega_{n}\right\}$ be Abelian differentials in $H^{1,0}(S)$ such that $c_{j}=\left[\operatorname{Re}\left(\omega_{j}\right)\right]$ for $j=1, \ldots, n$. Since $V$ is Hodge star-invariant, we get $\omega_{j} \in V^{1,0}$ for $j=1, \ldots, n$. Since 
$\left\{c_{1}, \ldots, c_{n}\right\}$ are symplectic-orthogonal and Hodge-orthonormal, the collection $\left\{\omega_{1}, \ldots, \omega_{n}\right\}$ is orthonormal, see (2.6). Complete the latter collection of Abelian differentials to an orthonormal basis $\left\{\omega_{1}, \ldots, \omega_{g}\right\}$ in $H^{1,0}(S)$. By construction $\left\{\omega_{n+1}, \ldots, \omega_{g}\right\}$ is an orthonormal basis in $\left(V^{\perp}\right)^{1,0}$. Finally, let $c_{j}=\left[\operatorname{Re}\left(\omega_{j}\right)\right]$ for $j=n+1, \ldots, g$. We have constructed a Hodgeorthonormal basis $\left\{c_{1}, \ldots, c_{g}\right\}$ of a Lagrangian subspace in $H^{1}(S, \mathbb{R})$ which completes the initial Hodge-orthonormal basis in the isotropic subspace $I_{n} \subset V$. By formula 2.49) we have

$$
\sum_{i=1}^{n} H_{\omega}^{\mathbb{R}}\left(c_{i}, c_{i}\right):=\sum_{i=1}^{n} H_{\omega}\left(\omega_{i}, \omega_{i}\right)=\sum_{i=1}^{n} \sum_{j=1}^{g}\left|B_{\omega}\left(\omega_{i}, \omega_{j}\right)\right|^{2}
$$

By Proposition 1 the matrix $B_{\omega}$ is block-diagonal in the chosen basis, so we obtain the following relations:

$$
\begin{gathered}
\Lambda_{1}^{V}+\cdots+\Lambda_{n}^{V}=\operatorname{Tr}\left(\left.H_{\omega}\right|_{V^{1,0}}\right)=\sum_{i=1}^{n} H_{\omega}\left(\omega_{i}, \omega_{i}\right)= \\
=\sum_{i=1}^{n} \sum_{j=1}^{g}\left|B_{\omega}\left(\omega_{i}, \omega_{j}\right)\right|^{2}=\sum_{i, j=1}^{n}\left|B_{\omega}\left(\omega_{i}, \omega_{j}\right)\right|^{2}
\end{gathered}
$$

Plugging the latter formula in definition 2.47) we finally obtain the following expression for $\Phi_{n}\left(\omega, I_{n}\right)$ :

$$
\Phi_{n}\left(\omega, I_{n}\right)=\sum_{i=1}^{n} \Lambda_{i}^{V}(\omega), \quad \text { for } \mu \text {-almost all } \omega \in \mathcal{H}_{g}^{(1)} .
$$

Since the function $\Phi_{n}$ has no dependence on the maximal isotropic subspace $I_{n} \subset V$ and the subbundle $V$ is $\operatorname{SL}(2, \mathbb{R})$-invariant, the statement follows from the variational formula given in Lemma 2.9 and mimics the proof of the Kontsevich formula (see Corollary 3.3 above and [F2], Corollary 5.3). Alternatively, the statement can be now immediately obtained from the more general formula (3.16) on partial sums of exponents in Corollary 3.2 above (see [F2], Corollary 5.5 for the proof).

\section{Degenerate Kontsevich-Zorich Spectrum}

In this section we collect several results which address the occurrence of zero Kontsevich-Zorich exponents. In particular, we prove that all the exponents are zero on a given $\operatorname{SL}(2, \mathbb{R})$-invariant subbundle if and only if the cocycle is isometric and that happens whenever the second fundamental form vanishes on that subbundle. In all known examples the vanishing of the second fundamental form can be derived from symmetries (automorphisms) of (almost) all Abelian differentials in the support of an $\operatorname{SL}(2, \mathbb{R})$ invariant measure. We conclude with a partial converse which gives a lower 
bound on the number of strictly positive exponents in terms of the rank of the second fundamental form.

4.1. Isometric subbundles. By the variational formulas, whenever the second fundamental form vanishes identically on any flow-invariant subbundle then the Kontsevich-Zorich cocycle acts isometrically, hence all of its exponents are zero. We prove below partial converse results in the special case of $\operatorname{SL}(2, \mathbb{R})$-invariant, Hodge star-invariant subbundles.

Lemma 4.1. Let $V \subset H_{\mathbb{R}}^{1}$ be a flow-invariant subbundle over a full measure set for a flow-invariant ergodic Borel probability measure $\mu$ on the moduli space $\mathcal{H}_{g}^{(1)}$ of normalized Abelian differentials. Consider the following two properties:

(1) the bilinear form $\left.B_{\omega}^{\mathbb{R}}\right|_{V}$ vanishes for $\mu$-almost all $\omega \in \mathcal{H}_{g}^{(1)}$;

(2) the restriction of the Kontsevich-Zorich cocycle to $V$ is isometric with respect to the Hodge norm.

Then, one has that (1) implies (2). Moreover, if one also assumes that $V$ is Hodge star-invariant, then (2) implies (1).

Proof. By Lemma 2.6, the real part of the bilinear form $B_{\omega}^{\mathbb{R}}$ gives the derivative of the Hodge inner product under the action of the KontsevichZorich cocycle at any $\omega \in \mathcal{H}_{g}^{(1)}$. Whenever $\left.B_{\omega}^{\mathbb{R}}\right|_{V}$ vanishes for $\mu$-almost all $\omega \in \mathcal{H}_{g}^{(1)}$, by continuity it vanishes identically on the support of the measure, and, hence, it follows from the variational formula (2.45) that the Kontsevich-Zorich cocycle acts isometrically on $V$ with respect to the Hodge inner product.

Let us show now that (2) implies (1) (assuming also that $V$ is Hodge star-invariant). By the variational formula (2.39) the real part of the bilinear form $B$ vanishes on $V^{1,0}$, i.e. $\operatorname{Re} B(\alpha, \beta)=0$ for all $\alpha, \beta \in V^{1,0}$. Note that, since $B_{\omega}$ is complex bilinear,

$$
B_{\omega}\left(e^{i \varphi} \alpha, e^{i \varphi} \beta\right)=e^{2 i \varphi} B_{\omega}(\alpha, \beta) .
$$

Since the Hodge star-invariance of $V$ implies that $V^{1,0}$ is a complex space, we see that if $\operatorname{Re} B(\alpha, \beta)=0$ for all $\alpha, \beta \in V^{1,0}$, then $B_{\omega}(\alpha, \alpha)=0$.

Remark 4.1. Under either condition (1) or condition (2) of Lemma 4.1 above, all Lyapunov exponents of the restriction of the Kontsevich-Zorich cocycle to $V$ are equal to zero. In fact, any isometric cocycle has a Lyapunov spectrum reduced to the single exponent zero.

Under the extra assumption that the invariant subbundle $V$ and the ergodic measure $\mu$ are invariant not only with respect to the Teichmüller flow, but with respect to the action of $\mathrm{SL}(2, \mathbb{R})$, one can prove a converse statement and prove that vanishing of all Lyapunov exponents of an invariant 
subbundle implies vanishing of the second fundamental form on this subbundle, see Theorem 3 below.

In the particular case that $V$ is Hodge star-invariant, the converse result becomes a straightforward corollary of the generalized Kontsevich formula (see formula (3.19) in Corollary 3.5).

Corollary 4.1. Let $V \subset H_{\mathbb{R}}^{1}$ be an $\mathrm{SL}(2, \mathbb{R})$-invariant, Hodge star-invariant subbundle over a full measure set for an $\mathrm{SL}(2, \mathbb{R})$-invariant ergodic Borel probability measure $\mu$ on the moduli space $\mathcal{H}_{g}^{(1)}$ of Abelian differentials. The following properties are equivalent:

(1) the bilinear form $\left.B_{\omega}^{\mathbb{R}}\right|_{V}$ vanishes for $\mu$-almost all $\omega \in \mathcal{H}_{g}^{(1)}$;

(2) the restriction of the Kontsevich-Zorich cocycle to $V$ is isometric with respect to the Hodge norm;

(3) the non-negative Lyapunov spectrum of $V$ has the form

$$
\lambda_{1}^{V, \mu}=\cdots=\lambda_{n}^{V, \mu}=0 .
$$

Proof. The first two statements are equivalent by Lemma 4.1. The second statement implies the third by the definition of Lyapunov exponents. All the above statements hold for any flow-invariant subbundle. If $V$ is $\operatorname{SL}(2, \mathbb{R})$ invariant and Hodge star-invariant, the third statement implies the first statement by the generalized Kontsevich formula. In fact, by that formula (see Corollary 3.5) the vanishing of all Lyapunov exponents of $V$ implies that

$$
\Lambda_{1}^{V}(\omega)=\cdots=\Lambda_{n}^{V}(\omega)=0, \quad \text { for } \mu \text {-almost all } \omega \in \mathcal{H}_{g}^{(1)} .
$$

It follows that $\left.H_{\omega}\right|_{V^{1,0}}$ vanishes on the support of the measure $\mu$ in $\mathcal{H}_{g}^{(1)}$. By formula (2.31) this implies that the bilinear forms $\left.B_{\omega}\right|_{V^{1,0}}$ and, thus, $\left.B_{\omega}^{\mathbb{R}}\right|_{V}$ also vanish for $\mu$-almost all $\omega \in \mathcal{H}_{g}^{(1)}$.

An important particular case of the above Corollary 4.1 is given below. In Appendix A we shall see other examples.

The Hodge bundle $H_{\mathbb{R}}^{1}$ over $\mathcal{H}_{g}^{(1)}$ splits into a direct sum of two subbundles. The first one (the tautological subbundle ) has dimension two; its fiber are spanned by cohomology classes $[\operatorname{Re}(\omega)]$ and $[\operatorname{Im}(\omega)] \in H^{1}(S, \mathbb{R})$. The second subbundle, $W$, is the orthogonal complement to the first one with respect to the symplectic intersection form (and with respect to the Hodge inner product) on the Hodge bundle $H_{\mathbb{R}}^{1}$. Clearly, both the tautological subbundle and its orthogonal complement $W$ are $\mathrm{SL}(2, \mathbb{R})$-invariant and Hodge star-invariant. In particular, for all $\omega \in \mathcal{H}_{g}^{(1)}$ the space $W_{\omega}^{1,0}$ is the orthogonal complement to $\omega$ in $H^{1,0}(S)$ with respect to the Hermitian form (2.1).

By Corollary 4.1 we have the following result (see Corollary 7.1 in [F3]): 
Corollary 4.2. Let $\mu$ be an $\mathrm{SL}(2, \mathbb{R})$-invariant ergodic Borel probability measure on the moduli space $\mathcal{H}_{g}^{(1)}$ of normalized Abelian differentials. The second fundamental form $B_{\omega}$ has rank equal to 1 for $\mu$-almost all $\omega \in \mathcal{H}_{g}^{(1)}$ if and only if all the non-trivial Lyapunov exponents of the KontsevichZorich cocycle with respect to $\mu$ vanish, that is, if and only if

$$
\lambda_{2}^{\mu}=\cdots=\lambda_{g}^{\mu}=0 .
$$

Proof. It can be verified explicitly that the Lyapunov spectrum of the tautological bundle is $\{1,-1\}$. It follows that the non-negative Lyapunov spectrum of its symplectic orthogonal complement $W \subset H_{\mathbb{R}}^{1}$ is $\left\{\lambda_{2}^{\mu}, \ldots, \lambda_{g}^{\mu}\right\}$. Since by definition $B_{\omega}(\omega, \omega)=1$, the rank of $B_{\omega}$ is 1 if and only if the rank of $\left.B_{\omega}\right|_{W^{1,0}}$ is zero, for all $\omega \in \mathcal{H}_{g}^{(1)}$. The statement then follows from Corollary 4.1 for the $\mathrm{SL}(2, \mathbb{R})$-invariant, Hodge star-invariant bundle $W \subset H_{\mathbb{R}}^{1}$.

4.2. A symmetry criterion. We recall below a simple symmetry criterion for the vanishing of the second fundamental form found in [F3], §7. Let $\omega \in \mathcal{H}_{g}^{(1)}$ be an Abelian differential on a Riemann surface $S$. Suppose that $S$ has a holomorphic automorphism $T$ and that the holomorphic 1-form $\omega$ is an eigenvector of the induced action $T^{*}: H^{1,0}(S) \rightarrow H^{1,0}(S)$. Denote by $u(T)$ the corresponding eigenvalue, $T^{*} \omega=u(T) \omega$. Note that the action $T^{*}: H^{1,0}(S) \rightarrow H^{1,0}(S)$ preserves the restriction of the Hermitian intersection form 2.1 which is positive-definite on $H^{1,0}(S)$, which implies that $|u(T)|=1$ and $\left.T^{*}\right|_{H^{1,0}(S)}$ is diagonalizable. Consider a basis $\left\{\omega_{1}, \ldots, \omega_{g}\right\}$ of eigenvectors of $T^{*}$ in $H^{1,0}(S)$ and denote the corresponding eigenvalues by $u_{1}(T), \ldots, u_{g}(T)$. The following statement is a simplified version of Lemma 7.2 in [F3].

Theorem 2. Let $\mathcal{M}$ be an $\mathrm{SL}(2, \mathbb{R})$-invariant suborbifold in some stratum of Abelian differentials in genus $g$. Let $\mathcal{M}$ be endowed with an ergodic probability measure. Suppose that almost every flat surface $(S, \omega)$ in $\mathcal{M}$ is endowed with a holomorphic automorphism $T: S \rightarrow S$, and that $\omega$ is an eigenvector of $T^{*}$ with an eigenvalue $u(T)$. Denote by $u_{1}(T), \ldots, u_{g}(T)$ all eigenvalues of $T^{*}: H^{1,0}(S) \rightarrow H^{1,0}(S)$.

If for all but one couple of indices $(i, j)$, where $1 \leq i \leq j \leq g$, one has $u_{i}(T) u_{j}(T) \neq u^{2}(T)$, then the rank of the bilinear form $B_{\omega}$ on $H^{1,0}$ is equal to 1 for all $\omega \in \mathcal{M}$, and, hence, all the non-trivial Lyapunov exponents of the Hodge bundle with respect to the Teichmüller geodesic flow on $\mathcal{M}$ vanish:

$$
\lambda_{2}=\cdots=\lambda_{g}=0 .
$$

Proof. Consider a holomorphic automorphism $T: S \rightarrow S$. For any two holomorphic differentials $\omega_{i}, \omega_{j}$ of our basis of eigenvectors of the linear 
map $T^{*}$ in $H^{1,0}(S)$, by definition (2.11) of the form $B_{\omega}$ and by change of coordinates, we get

$$
\begin{aligned}
B_{\omega}\left(\omega_{i}, \omega_{j}\right) & =\frac{i}{2} \int_{S} \frac{\omega_{i} \omega_{j}}{\omega} \bar{\omega}=\frac{i}{2} \int_{S} \frac{T^{*} \omega_{i} T^{*} \omega_{j}}{T^{*} \omega} T^{*} \bar{\omega}= \\
& =\frac{i}{2} \int_{S} \frac{u_{i}(T) u_{j}(T)}{u^{2}(T)} \frac{\omega_{i} \omega_{j}}{\omega} \bar{\omega}=\frac{u_{i}(T) u_{j}(T)}{u^{2}(T)} B_{\omega}\left(\omega_{i}, \omega_{j}\right) .
\end{aligned}
$$

Hence, for every pair of indices $i, j$ such that $u_{i}(T) u_{j}(T) \neq u^{2}(T)$, the element $B_{\omega}\left(\omega_{i}, \omega_{j}\right)$ of the matrix of the form $B_{\omega}$ is equal to zero. The result now follows from Corollary 4.2 .

In Appendix A (more precisely, Subsection A.2 below), we will give an application of this symmetry criterion (for the vanishing of exponents) provided by Theorem 2 in a particular interesting case (of an arithmetic Teichmüller disk of a square-tiled cyclic cover in genus 4).

4.3. The central Oseledets subbundle. An important example of a Hodge star-invariant subspace is given by the kernel of the second fundamental form. In fact, the following elementary result holds. For any $(S, \omega) \in \mathcal{H}_{g}$, let $\operatorname{Ann}\left(B_{\omega}^{\mathbb{R}}\right)$ denote the kernel of the form $B_{\omega}^{\mathbb{R}}$ on $H^{1}(S, \mathbb{R})$, that is:

$$
\operatorname{Ann}\left(B_{\omega}^{\mathbb{R}}\right):=\left\{c \in H^{1}(S, \mathbb{R}) \mid B_{\omega}^{\mathbb{R}}\left(c, c^{\prime}\right)=0 \quad \forall c^{\prime} \in H^{1}(S, \mathbb{R})\right\} .
$$

Lemma 4.2. For any $(S, \omega) \in \mathcal{H}_{g}$ the subspace $\operatorname{Ann}\left(B_{\omega}^{\mathbb{R}}\right)$ in $H^{1}(S, \mathbb{R})$ is Hodge star-invariant. Moreover, $\left(\operatorname{Ann}\left(B_{\omega}^{\mathbb{R}}\right)\right)^{1,0}=\operatorname{Ann} B_{\omega}$.

Proof. Let $c_{1}, c_{2} \in H^{1}(S, \mathbb{R})$ be any two cohomology classes, and let $\omega_{1}, \omega_{2}$ be holomorphic 1-forms such that $c_{1}=\left[\operatorname{Re}\left(\omega_{1}\right)\right]$ and $c_{2}=\left[\operatorname{Re}\left(\omega_{2}\right)\right]$. By definition $* c_{1}=\left[\operatorname{Im}\left(\omega_{1}\right)\right]$, so we have $* c_{1}=\left[\operatorname{Re}\left(-i \omega_{1}\right)\right]$. Thus, by the definition of $B_{\omega}^{\mathbb{R}}$ given in Section 2.5 and by bilinearity of the form $B_{\omega}$ defined in 2.11) one gets

$$
B_{\omega}^{\mathbb{R}}\left(* c_{1}, c_{2}\right):=B_{\omega}\left(-i \omega_{1}, \omega_{2}\right)=-i B_{\omega}\left(\omega_{1}, \omega_{2}\right)=-i B_{\omega}^{\mathbb{R}}\left(c_{1}, c_{2}\right) .
$$

Hence, if for some $c_{1} \in H^{1}(S, \mathbb{R})$ one has the identity $B_{\omega}^{\mathbb{R}}\left(c_{1}, c_{2}\right)=0$ for all $c_{2} \in H^{1}(S, \mathbb{R})$, one also has $B_{\omega}^{\mathbb{R}}\left(* c_{1}, c_{2}\right)=0$ for all $c_{2} \in H^{1}(S, \mathbb{R})$. The last statement is a direct corollary of the definition of $B_{\omega}^{\mathbb{R}}$.

Another remarkable, however simple, property of the bundle $\operatorname{Ann}\left(B_{\omega}^{\mathbb{R}}\right)$ is described below:

Lemma 4.3. For any flow-invariant (resp., $\mathrm{SL}(2, \mathbb{R})$-invariant) measurable subbundle $V \subset \operatorname{Ann}\left(B_{\omega}^{\mathbb{R}}\right)$ the Hodge orthogonal splitting $H_{\mathbb{R}}^{1}=V \oplus V^{\perp}$ is flow-invariant (resp., $\mathrm{SL}(2, \mathbb{R})$-invariant). 
Proof. By the variational formula (2.39) of Lemma2.6 for the Hodge innner product, the condition $V \subset \operatorname{Ann}\left(B_{\omega}^{\mathbb{R}}\right)$ implies that the Hodge product $(v, w)$ is constant for any parallel (locally constant) sections $v \in V$ and $w \in H_{\mathbb{R}}^{1}$. In particular, the equation $(v, w)=0$ is invariant under parallel transport.

We prove below our strongest result on the central Oseledets bundle.

Theorem 3. Let $\mu$ be a flow-invariant ergodic probability measure on the moduli space $\mathcal{H}_{g}^{(1)}$ of normalized Abelian differentials.

If $V \subset H_{\mathbb{R}}^{1}$ is a flow-invariant subbundle of the Hodge bundle such that $\operatorname{Ann}\left(B^{\mathbb{R}} \mid V\right)$ is $\mu$-almost everywhere flow-invariant, then the dimension $\operatorname{dim} \operatorname{Ann}\left(B^{\mathbb{R}} \mid V_{\omega}\right)$ is the same for $\mu$-almost all $\omega \in \mathcal{H}_{g}^{(1)}$ (so $\operatorname{Ann}\left(B^{\mathbb{R}} \mid V\right)$ defines a vector bundle over the support of $\mu$ ) and

$$
\operatorname{Ann}\left(B^{\mathbb{R}} \mid V\right) \subseteq E_{(0)}^{\mu} \cap V \quad(\mu \text {-almost everywhere }) .
$$

If $\mu$ is $\mathrm{SL}(2, \mathbb{R})$-invariant and $W$ is any $\mathrm{SL}(2, \mathbb{R})$-invariant measurable subbundle of the Oseledets (measurable) bundle $E_{(0)}^{\mu}$, then

$$
W \subseteq \operatorname{Ann}\left(B^{\mathbb{R}}\right) \quad(\mu \text {-almost everywhere }) .
$$

In particular, if $\mu$ is $\mathrm{SL}(2, \mathbb{R})$-invariant and $E_{(0)}^{\mu} \cap V$ is $\mu$-almost everywhere $\operatorname{SL}(2, \mathbb{R})$-invariant, then $E_{(0)}^{\mu} \cap V \subseteq \operatorname{Ann}\left(B^{\mathbb{R}}\right) \quad$ ( $\mu$-almost everywhere).

Consequently, if $\mu$ is $\mathrm{SL}(2, \mathbb{R})$-invariant and $V \subset H_{\mathbb{R}}^{1}$ is a Hodge starinvariant and $\mathrm{SL}(2, \mathbb{R})$-invariant subbundle of the Hodge bundle such that $\operatorname{Ann}\left(B^{\mathbb{R}} \mid V\right)$ is $\mu$-almost everywhere flow-invariant and $E_{(0)}^{\mu} \cap V$ is $\mu$-almost everywhere $\mathrm{SL}(2, \mathbb{R})$-invariant, then

$$
E_{(0)}^{\mu} \cap V=\operatorname{Ann}\left(B^{\mathbb{R}} \mid V\right)=\operatorname{Ann}\left(B^{\mathbb{R}}\right) \cap V \quad(\mu \text {-almost everywhere }) .
$$

Proof. Since $\operatorname{dim} \operatorname{Ann}\left(B^{\mathbb{R}} \mid V\right)$ is, by hypothesis, a flow-invariant integervalued function on $\mathcal{H}_{g}^{(1)}$, the ergodicity of $\mu$ implies that it is constant $\mu$ almost everywhere. (The dimension can jump and become larger, say, on suborbifolds of nontrivial codimension). Thus, $\operatorname{Ann}\left(B^{\mathbb{R}} \mid V\right)$ defines a flowinvariant vector bundle over the support of $\mu$ and we can apply Lemma 4.1 to this bundle to prove the first statement.

The last statement is a trivial combination of the first two, so the essential part of the Theorem is the second statement, which is proved below.

Let $\mathcal{N}_{k}^{W}(\mu)$ be the space of all probability measures on the Grassmannian bundle $G_{k}(W)$ of $k$-dimensional isotropic subspaces projecting on $\mathcal{H}_{g}^{(1)}$ to some $\bar{\mu}$ absolutely continuous with respect to $\mu$. Let us denote by $2 p$ the rank of the restriction of the symplectic form to the subbundle $W$ and by $l$ the difference $l=\operatorname{dim} W-p$. By Lemma 3.2, for any $k \in \mathbb{N}$ satisfying the relations $1 \leq k \leq l$, the space $\mathcal{N}_{k}^{W}(\mu)$ is non-empty. Let $\mathcal{O}_{k}^{W}(\mu) \subset \mathcal{N}_{k}^{W}(\mu)$ 
be the subset of all $\mathrm{SO}(2, \mathbb{R})$-invariant measures. As we have already seen in section 3.2, since $\mathrm{SO}(2, \mathbb{R})$ is a compact amenable group, the set $\mathcal{O}_{k}^{W}(\mu)$ is non-empty whenever $\mathcal{N}_{k}^{W}(\mu)$ is non-empty: the $\mathrm{SO}(2, \mathbb{R})$-average of any measure in $\mathcal{N}_{k}^{W}(\mu)$ is a measure in $\mathcal{O}_{k}^{W}(\mu)$.

Since by assumption $W \subset E_{(0)}^{\mu}$, all the Lyapunov exponents of the restriction of Kontsevich-Zorich cocycle to $W$ are zero, hence by the Oseledets theorem the average Lyapunov exponent $\Lambda^{(k)}(\nu)$ of any probability measure $\nu \in \mathcal{N}_{k}^{W}(\mu)$ is equal to zero. By Theorem 1 it follows that, for any measure $\nu \in \mathcal{O}_{k}^{W}(\mu)$ and for any weak limit $\hat{\nu} \in I(\nu)$,

$$
0=\int_{G_{k}(W)} \Phi_{k} d \hat{\nu} .
$$

Since $\Phi_{k}$ is by definition non-negative, the above formula implies that $\Phi_{k}$ vanishes $\hat{\nu}$-almost everywhere. Hence, for $\hat{\nu}$-almost all $\left(\omega, I_{k}\right) \in G_{k}(W)$ we get the following conclusion. By applying the identity in the middle of formula (2.51) to any Lagrangian Hodge-orthonormal completion $\left\{c_{1}, \ldots, c_{k}, c_{k+1}, \ldots, c_{g}\right\}$ of a Hodge-orthonormal basis $\left\{c_{1}, \ldots, c_{k}\right\}$ of the $k$-dimensional isotropic subspace $I_{k} \subset W_{\omega}$ we get

$$
0=\Phi_{k}\left(\omega, I_{k}\right)=\sum_{i=1}^{k} \sum_{j=1}^{g}\left|B_{\omega}\left(\omega_{i}, \omega_{j}\right)\right|^{2}+\sum_{i=1}^{k} \sum_{j=k+1}^{g}\left|B_{\omega}\left(\omega_{i}, \omega_{j}\right)\right|^{2} .
$$

By the definition (2.33) of the form $B^{\mathbb{R}}$, it follows that $B_{\omega}^{\mathbb{R}}\left(c_{i}, c_{j}\right)=0$ for all $i \in\{1, \ldots, k\}$ and $j \in\{1, \ldots, g\}$, or, equivalently, that $I_{k} \subset \operatorname{Ann}\left(B_{\omega}^{\mathbb{R}}\right)$. Thus we have proved that, for any Borel probability measure $\nu \in \mathcal{O}_{k}^{W}(\mu)$ and for any weak limit $\hat{\nu} \in I(\nu)$,

$$
I_{k} \subset \operatorname{Ann}\left(B_{\omega}^{\mathbb{R}}\right), \quad \text { for } \hat{\nu} \text {-almost all }\left(\omega, I_{k}\right) \in G_{k}(W) .
$$

For every Borel probability measure $\nu \in \mathcal{O}_{k}^{W}(\mu)$, we then define, over the support of the measure $\mu$ on $\mathcal{H}_{g}^{(1)}$, a flow-invariant Borel measurable subbundle $F(\nu) \subset W$ as follows. For $\mu$-almost all Abelian differentials $\omega \in \mathcal{H}_{g}^{(1)}$ we let the fiber $F_{\omega}(\nu)$ be the linear span of all isotropic subspaces $I_{k} \subset W_{\omega}$ such that $\left(\omega, I_{k}\right)$ belongs to the essential support of at least one measure $\hat{\nu} \in I(\nu)$ on the Grassmannian $G_{k}(W)$. Since $I(\nu)$ is a compact set of probability measures, and, by Rokhlin's disintegration theorem [Ro], for each $\hat{\nu} \in I(\nu)$, the conditional measures $\hat{\nu}_{\omega}$ of $\hat{\nu}$ on the fibers $G_{k}\left(H_{\mathbb{R}}^{1}\right)_{\omega}$ depend measurably on $\omega \in \mathcal{H}_{g}^{(1)}$, one can check that $F_{\omega}(\nu)$ depends measurably on $\omega \in \mathcal{H}_{g}^{(1)}$. By construction, since all measures $\hat{\nu} \in I(\nu)$ are flowinvariant, the family of subspaces $F_{\omega}(\nu)$ is defined $\mu$-almost everywhere and flow-invariant. Since the measure $\mu$ is ergodic, this implies that the dimension $\operatorname{dim} F_{\omega}(\nu)$ is $\mu$-almost everywhere constant, hence $F(\nu) \subset W$ is a $\mu$-measurable flow-invariant subbundle. By construction, every such $F(\nu)$ 
is a subbundle of $\operatorname{Ann}\left(B^{\mathbb{R}}\right)$, since at $\mu$-almost all $\omega \in \mathcal{H}_{g}^{(1)}$ the fiber $F_{\omega}(\nu)$ is spanned by subspaces $I_{k}$ of $\operatorname{Ann}\left(B_{\omega}^{\mathbb{R}}\right)$.

We then define, for every $1 \leq k \leq l$ one more flow-invariant measurable subbundle $F_{k}^{\mu} \subset W$ over the support of $\mu$ on $\mathcal{H}_{g}^{(1)}$. For $\mu$-almost any $\omega \in \mathcal{H}_{g}^{(1)}$, the fiber $\left(F_{k}^{\mu}\right)_{\omega}$ is defined as a linear span of the family of vector spaces $\left\{F_{\omega}(\nu)\right\}_{\nu \in \mathcal{O}_{k}^{W}(\mu)}$. Finally, we let $F^{\mu} \subset W$ be the flow-invariant measurable subbundle, defined $\mu$-almost everywhere, such that the fiber $F_{\omega}^{\mu}$ over $\mu$-almost any $\omega \in \mathcal{H}_{g}^{(1)}$ is the linear span of vector subspaces $\left\{\left(F_{k}^{\mu}\right)_{\omega}\right\}_{1 \leq k \leq l}$. As above, since $\mu$ is ergodic, the bundles $F_{k}^{\mu}$ and $F^{\mu}$ are indeed measurable subbundles. By construction, the measurable bundle $F^{\mu}$ is a subbundle of $\operatorname{Ann}\left(B^{\mathbb{R}}\right)$, since its fiber at $\mu$-almost any Abelian differential $\omega \in \mathcal{H}_{g}^{(1)}$ is spanned by subspaces of $\operatorname{Ann}\left(B_{\omega}^{\mathbb{R}}\right)$.

Let us argue by contradiction. Let us assume that the subset $\mathcal{P} \subset \mathcal{H}_{g}^{(1)}$ of all $\omega \in \mathcal{H}_{g}^{(1)}$ such that $W_{\omega} \nsubseteq \operatorname{Ann}\left(B_{\omega}^{\mathbb{R}}\right)$ has positive measure (with respect to the $\mathrm{SL}(2, \mathbb{R})$-invariant ergodic probability measure $\mu$ on $\left.\mathcal{H}_{g}^{(1)}\right)$. Since the subbundles $\operatorname{Ann}\left(B^{\mathbb{R}}\right)$ and $W$ are $\mathrm{SO}(2, \mathbb{R})$-invariant, it follows that the set $\mathcal{P}$ is $\operatorname{SO}(2, \mathbb{R})$-invariant. For $\mu$-almost all $\omega \in \mathcal{H}_{g}^{(1)}$, let $\mathrm{SO}(2, \mathbb{R}) F_{\omega}^{\mu} \subset W_{\omega}$ denote the smallest $\mathrm{SO}(2, \mathbb{R})$-invariant linear subspace which contains the subspace $F_{\omega}^{\mu} \subset W_{\omega}$. The vector space $\mathrm{SO}(2, \mathbb{R}) F_{\omega}^{\mu}$ can be defined as the intersection of the (non-empty) family of all $\mathrm{SO}(2, \mathbb{R})$-invariant subspaces of the vector space $W_{\omega}$ which contain $F_{\omega}^{\mu}$ or , equivalently, as the span of the union of the family $\left\{F_{\omega^{\prime}}^{\mu} \mid \omega^{\prime} \in \mathrm{SO}(2, \mathbb{R}) \cdot \omega\right\}$. By construction $F_{\omega}^{\mu} \subset \operatorname{Ann}\left(B_{\omega}^{\mathbb{R}}\right)$, hence $\operatorname{SO}(2, \mathbb{R}) F_{\omega}^{\mu} \subset \operatorname{Ann}\left(B_{\omega}^{\mathbb{R}}\right)$, which implies that $\mathrm{SO}(2, \mathbb{R}) F_{\omega}^{\mu} \neq W_{\omega}$, for all $\omega \in \mathcal{P}$. Note that it is not restrictive to assume that the dimension of the vector space $\operatorname{SO}(2, \mathbb{R}) F_{\omega}^{\mu}$ is constant for all $\omega \in \mathcal{P}$. In fact, the positive measure set $\mathcal{P}$ has a finite partition

$$
\mathcal{P}:=\bigcup_{d=1}^{\operatorname{dim}(W)-1}\left\{\omega \in \mathcal{P} \mid \operatorname{dim}\left(\operatorname{SO}(2, \mathbb{R}) F_{\omega}^{\mu}\right)=d\right\}
$$

hence at least one of these sets has positive measure. We can therefore assume that the collection of vector spaces $\left\{\mathrm{SO}(2, \mathbb{R}) F_{\omega}^{\mu} \mid \omega \in \mathcal{P}\right\}$ forms a proper measurable $\mathrm{SO}(2, \mathbb{R})$-invariant subbundle $\mathrm{SO}(2, \mathbb{R}) F^{\mu}$ of the restriction $W \mid \mathcal{P}$ of the subbundle $W$ to $\mathcal{P}$. By the above construction, it follows that the bundle $\left(\mathrm{SO}(2, \mathbb{R}) F^{\mu}\right)^{\perp} \cap W \mid \mathcal{P}$ is a non-trivial $\mathrm{SO}(2, \mathbb{R})$-invariant subbundle of the restriction of the subbundle $\left(F^{\mu}\right)^{\perp} \cap W$ to $\mathcal{P}$.

We claim that there exists $k_{0} \in\{1, \ldots, l\}$ such that for all $k \leq k_{0}$ there exists an $\mathrm{SO}(2, \mathbb{R})$-invariant probability measure $\nu_{\mathcal{P}}^{*} \in \mathcal{O}_{k}^{W}(\mu)$ essentially supported on the Grassmannian $G_{k}\left(\left(F^{\mu}\right)^{\perp} \cap W\right)$. In fact, let $\mu_{\mathcal{P}}$ be the restriction of the measure $\mu$ on $\mathcal{H}_{g}^{(1)}$ to the positive measure set $\mathcal{P}$, normalized 
to have unit total mass. There exists $k_{0} \in \mathbb{N} \backslash\{0\}$ such that the Grassmannian of $k_{0}$-dimensional isotropic subspaces of the (non-trivial) subbundle $\left(\mathrm{SO}(2, \mathbb{R}) F^{\mu}\right)^{\perp} \cap W_{\mathcal{P}}$ is non-empty. By Lemma 3.2. for any $k \leq k_{0}$ there exists a probability measure $\nu_{\mathcal{P}}$ on $G_{k}\left(H^{1}\right)$ essentially supported on $G_{k}\left(\left(\mathrm{SO}(2, \mathbb{R}) F^{\mu}\right)^{\perp} \cap W\right)$, which projects onto the probability measure $\mu_{\mathcal{P}}$ on $\mathcal{H}_{g}^{(1)}$ under the canonical projection. Let $\nu_{\mathcal{P}}^{*}$ be the $\mathrm{SO}(2, \mathbb{R})$-average of the measure $\nu_{\mathcal{P}}$. By construction $\nu_{\mathcal{P}}^{*}$ belongs to the set $\mathcal{O}_{k}^{W}(\mu)$ : in fact, it is $\mathrm{SO}(2, \mathbb{R})$-invariant, it projects onto the probability measure $\mu_{\mathcal{P}}$, which is absolutely continuous with respect to $\mu$ on $\mathcal{H}_{g}^{(1)}$, and it is essentially supported on $G_{k}\left(\left(F^{\mu}\right)^{\perp} \cap W\right)$. The above claim is therefore proved.

Since $F^{\mu} \subset \operatorname{Ann}\left(B^{\mathbb{R}}\right)$ and by construction it is flow-invariant, by Lemma 4.3 there is a flow-invariant Hodge-orthogonal splitting

$$
H_{\mathbb{R}}^{1}=F^{\mu} \oplus\left(F^{\mu}\right)^{\perp} .
$$

It follows that, on the one hand, $F\left(\nu_{\mathcal{P}}^{*}\right) \subset\left(F^{\mu}\right)^{\perp}$ by construction, since $\nu_{\mathcal{P}}^{*}$ is essentially supported on $G_{k}\left(\left(F^{\mu}\right)^{\perp} \cap W\right)$ and the bundle $\left(F^{\mu}\right)^{\perp} \cap W$ is flow invariant; on the other hand, $F\left(\nu_{\mathcal{P}}^{*}\right) \subset F^{\mu}$ by the definition of the bundle $F^{\mu}$ given above. Of course, this is a contradiction since $F^{\mu} \cap\left(F^{\mu}\right)^{\perp}=\{0\}$. The argument is therefore complete.

Remark 4.2. Concerning the invariance assumptions (under Teichmüller flow and/or $\operatorname{SL}(2, \mathbb{R})$ ) in the previous theorem, we would like to stress out that they are really necessary. More precisely, while in Appendix A below we introduce a class of ergodic $\operatorname{SL}(2, \mathbb{R})$-invariant probability measures supported on the $\mathrm{SL}(2, \mathbb{R})$-orbit of square-tiled cyclic covers such that we can show $E_{(0)}^{\mu}=\operatorname{Ann}\left(B^{\mathbb{R}}\right)$ is $\operatorname{SL}(2, \mathbb{R})$-invariant (see Theorem 7 below), we construct in Appendix B below an example of a closed $\operatorname{SL}(2, \mathbb{R})$-invariant locus $Z$ supporting an ergodic $\mathrm{SL}(2, \mathbb{R})$-invariant probability measure such that $E_{(0)}^{\mu}$ is not $\mathrm{SL}(2, \mathbb{R})$-invariant, $\operatorname{Ann}\left(B^{\mathbb{R}}\right)$ is not Teichmüller invariant and hence $E_{(0)}^{\mu} \neq \operatorname{Ann}\left(B^{\mathbb{R}}\right)$ even though they have the same dimension! In other words, although $\operatorname{Ann}\left(B^{\mathbb{R}}\right)$ doesn't coincide with $E_{(0)}^{\mu}$ in the case of the locus $Z$, the number of zero exponents is still predicted by the corank of $B^{\mathbb{R}}$. Partly motivated by the features of these examples, we pose below the problem to establish whether the corank of $B^{\mathbb{R}}$ always give the correct number of central exponents (see Problem 1 below).

Note that invariance of a Hodge star-invariant vector subbundle $V \subset H_{\mathbb{R}}^{1}$ of the Hodge bundle under $\mathrm{SL}(2, \mathbb{R})$ does not imply invariance of the corresponding subbundle $V^{1,0} \subset H^{1,0}$ under $\operatorname{SL}(2, \mathbb{R})$ or under the flow. For example, the tautological bundle, spanned by $[\operatorname{Re} \omega]$ and $[\operatorname{Im} \omega]$ is $\operatorname{SL}(2, \mathbb{R})$ invariant, while the line bundle $\mathbb{C} \cdot \omega \subset H^{1,0}$ is not. The Lemma below shows that $\operatorname{Ann}\left(B^{\mathbb{R}}\right)$ is special in this sense. 
Proposition 2. Assume that the kernel $\operatorname{Ann}\left(B^{\mathbb{R}}\right)$ of the bilinear form $B^{\mathbb{R}}$ on $H_{\mathbb{R}}^{1}$ is invariant over an open interval $\left.U=\right]-\varepsilon, \varepsilon[$ along the trajectory of the Teichmüller flow passing through $\left(S_{0}, \omega_{0}\right) \in \mathcal{H}_{g}^{(1)}$. Then the kernel $\operatorname{Ann}(B)$ of the form $B$ on $H^{1,0}$ is also invariant over $U$.

An analogous statement holds when $U$ is replaced by a small open ball in $\mathrm{SL}(2, \mathbb{R})$ containing the identity element, or by a small open neighborhood of the initial point $\left(S_{0}, \omega_{0}\right)$ in the Teichmüller disc of $\left(S_{0}, \omega_{0}\right)$.

Proof. Since by Lemma 4.2 the subspace $V:=\operatorname{Ann}\left(B_{\omega}^{\mathbb{R}}\right)$ is Hodge starinvariant, the subspace $V_{\mathbb{C}}$ is invariant over the interval $U$ and admits a (a priori non-invariant) decomposition $V_{\mathbb{C}}=V_{t}^{1,0} \oplus V_{t}^{0,1}$, for all $t \in U$. By Lemma 4.2 $V_{t}^{1,0}=\operatorname{Ann}\left(B_{\omega_{t}}\right)$, hence by the definition (2.7) of the second fundamental form the projection of the covariant derivative $D_{H^{1}}(\omega)$ onto $V_{t}^{0,1}$ vanishes for every $\omega \in V_{t}^{1,0}$ and for all $t \in U$. It follows that the subspace $V_{t}^{1,0}$ is constant over $t \in U$, as stated.

4.4. Non-vanishing of the Kontsevich-Zorich exponents. In this subsection we prove a general lower bound on the number of strictly positive Lyapunov exponents of the Kontsevich-Zorich cocycle in terms of the rank of $B$. Such a bound is considerably weaker than a sharp estimate (roughly by a factor 2) and no upper bound other than Lemma 4.1 is known.

Theorem 4. Let $\mu$ be any $\mathrm{SL}(2, \mathbb{R})$-invariant ergodic probability measure on the moduli space of Abelian differentials. Let $V \subset H_{\mathbb{R}}^{1}$ be any Hodge star-invariant, $\mathrm{SL}(2, \mathbb{R}$ )-invariant subbundle (of dimension $n \in \mathbb{N}$ ) defined $\mu$-almost everywhere. If for some $k \in\{1, \ldots, n-1\}$ the bottom $n-k$ exponents of the restriction of the Kontsevich-Zorich cocycle to $V$ with respect to the measure $\mu$ vanish, that is,

$$
\lambda_{k+1}^{V, \mu}=\cdots=\lambda_{n}^{V, \mu}=0,
$$

then the rank of the bilinear form $B \mid V_{\omega}^{1,0}$ satisfies the inequality:

$$
\operatorname{rank}\left(B \mid V_{\omega}^{1,0}\right) \leq 2 k, \quad \text { for } \mu \text {-almost all } \omega \in \mathcal{H}_{g}^{(1)} .
$$

Proof. The proof follows closely the argument given in [[F2], Corollary 5.4, which was stated for the Hodge bundle with respect to the canonical absolutely continuous invariant measures only. In that case, the bilinear form $B$ has maximal rank (equal to $g$ ) on a subset of positive (Lebesgue) measure on each connected component of every stratum, as proved in [F2], §4, which implies that

$$
\lambda_{1}^{\mu}>\lambda_{2}^{\mu} \geq \cdots \geq \lambda_{[(g+1) / 2]}^{\mu}>0 .
$$

The generalized argument proceeds as follows. If all Lyapunov exponents vanish, then the $\operatorname{rank}\left(B \mid V_{\omega}^{1,0}\right)$ also vanishes by Corollary 4.1, hence the 
statement holds in this case. It follows that without loss of generality we may assume that $\lambda_{k}^{V, \mu}>0$ : if this assumption only holds for a smaller value of $k \in\{1, \ldots, n-1\}$, we would prove a statement even stronger than the claim.

Let us assume then that $\lambda_{k}^{V, \mu}>\lambda_{k+1}^{V, \mu}=0$. We are in the setting of Corollary 3.1 and we can apply formula (3.15). For $\mu$-almost all $\omega \in \mathcal{H}_{g}^{(1)}$, let $\left\{c_{1}, \ldots, c_{k}\right\} \subset V^{+}(\omega)$ be a Hodge orthonormal basis. Let $\left\{c_{1}, \ldots, c_{g}\right\} \subset$ $H^{1}(S, \mathbb{R})$ be a completion of the system $\left\{c_{1}, \ldots, c_{k}\right\}$ to a orthonormal basis of any Lagrangian subspace of $H^{1}(S, \mathbb{R})$. By formula (3.15) and by the definition of the function $\Phi_{k}$ in formula (2.46) we have

$$
\begin{aligned}
\lambda_{1}^{V, \mu}+\cdots+\lambda_{k}^{V, \mu} & =\int_{\mathcal{H}_{g}^{(1)}} \Phi_{k}\left(\omega, V_{k}^{+}(\omega)\right) d \mu(\omega)= \\
& =\int_{\mathcal{H}_{g}^{(1)}}\left(\sum_{i=1}^{g} \Lambda_{i}(\omega)-\sum_{i, j=k+1}^{g}\left|B_{\omega}^{\mathbb{R}}\left(c_{i}, c_{j}\right)\right|^{2}\right) d \mu(\omega) .
\end{aligned}
$$

Since $V$ is Hodge star-invariant, by the reducibility of the second fundamental form (see Proposition 1) it follows that

$$
\lambda_{1}^{V, \mu}+\cdots+\lambda_{k}^{V, \mu}=\int_{\mathcal{H}_{g}^{(1)}}\left(\sum_{i=1}^{n} \Lambda_{i}^{V}(\omega)-\sum_{i, j=k+1}^{n}\left|B_{\omega}^{\mathbb{R}}\left(c_{i}, c_{j}\right)\right|^{2}\right) d \mu(\omega) .
$$

By the generalized Kontsevich formula (see Corollary 3.5) we get

$$
\left(\lambda_{1}^{V, \mu}+\cdots+\lambda_{k}^{V, \mu}\right)+\left(\lambda_{k+1}^{V, \mu}+\cdots+\lambda_{n}^{V, \mu}\right)=\int_{\mathcal{H}_{g}^{(1)}} \sum_{i=1}^{n} \Lambda_{i}^{V}(\omega) d \mu .
$$

Since $\lambda_{k+1}^{V, \mu}=\cdots=\lambda_{n}^{V, \mu}=0$ the above two expressions coincide, which implies that

$$
\int_{\mathcal{H}_{g}^{(1)}} \sum_{i, j=k+1}^{n}\left|B_{\omega}^{\mathbb{R}}\left(c_{i}, c_{j}\right)\right|^{2} d \mu(\omega)=0 .
$$

This means that $\mu$-almost everywhere we have $B_{\omega}^{\mathbb{R}}\left(c_{i}, c_{j}\right)=0$ for any pair $(i, j)$ such that $i, j \geq k+1$. Thus, for $\mu$-almost all $\omega \in \mathcal{H}_{g}^{(1)}$ there exists an orthonormal basis $\left\{\omega_{1}, \ldots, \omega_{n}\right\}$ of the subspace $V_{\omega}^{1,0}$ of holomorphic differentials on the Riemann surface $S$ such that

$$
B_{\omega}\left(\omega_{i}, \omega_{j}\right)=0, \quad \text { for all } i, j \in\{k+1, \ldots, n\} .
$$

Hence, the matrix of the form $B \mid V_{\omega}^{1,0}$ with respect to the basis $\left\{\omega_{1}, \ldots, \omega_{n}\right\}$ has a $(n-k) \times(n-k)$ zero diagonal block. It follows that $B \mid V_{\omega}^{1,0}$ has rank at most $2 k$. 
Corollary 4.3 (see [[F2], Cor. 5.4). Let $\mu$ be any $\mathrm{SL}(2, \mathbb{R})$-invariant ergodic probability measure on the moduli space of Abelian differentials. Iffor some $k \in\{1, \ldots, g-1\}$ the bottom $g-k$ exponents of the Kontsevich-Zorich cocycle with respect to the measure $\mu$ vanish, that is,

$$
\lambda_{k+1}^{\mu}=\cdots=\lambda_{g}^{\mu}=0 .
$$

then the rank of the bilinear form $B_{\omega}$ on $H^{1,0}$ satisfies the inequality:

$$
\operatorname{rank}\left(B_{\omega}\right) \leq 2 k, \quad \text { for } \mu \text {-almost all } \omega \in \mathcal{H}_{g}^{(1)} .
$$

Remark 4.3. In [F3] the first author introduced analytic subvarieties $\mathcal{R}_{g}^{(1)}(k)$ of the moduli space $\mathcal{H}_{g}^{(1)}$, defined as follows:

$$
\mathcal{R}_{g}^{(1)}(k):=\left\{\omega \in \mathcal{H}_{g}^{(1)} \mid \operatorname{rank}\left(B_{\omega}\right) \leq k\right\}, \quad \text { where } k \in\{1, \ldots, g-1\} .
$$

In terms of such subvarieties Theorem 4 can be formulated as follows: if the support of the $\operatorname{SL}(2, \mathbb{R})$-invariant measure $\mu$ on $\mathcal{H}_{g}^{(1)}$ is not contained in the subvariety $\mathcal{R}_{g}^{(1)}(2 k)$, then formula (4.3) does not hold, that is,

$$
\lambda_{1}^{\mu}>\lambda_{2}^{\mu} \geq \cdots \geq \lambda_{k+1}^{\mu}>0 .
$$

Remark 4.4. A lower bound for the number of strictly positive exponents which holds in general for $\mathrm{SL}(2, \mathbb{R})$-invariant probability measures supported on regular orbifolds is proved in [EKZ1]. Such a bound cannot be derived from the above Corollary 4.3 without assumptions on the minimal rank of the fundamental form $B$ on the moduli space $\mathcal{H}_{g}^{(1)}$. However, to the authors best knowledge, lower bounds on the rank of the second fundamental forms for $g \geq 5$ are not available. It could be conjectured that it grows linearly with respect to the genus of the surface.

Remark 4.5. The argument given in the proof of Theorem 4 is not sufficient to prove the non-vanishing of all the Kontsevich-Zorich exponents. In fact, any improvement on Theorem 4 based on the formulas for sums of Lyapunov exponents, given in Corollary 3.2, seems to require some control a priori on the position of the unstable bundle $E_{g}^{+}$of the cocycle on a set of positive measure of Abelian differentials. In the case of a canonical absolutely continuous invariant measure such a set can be found near the boundary of the moduli space and the full non-vanishing of the Lyapunov spectrum can thus be proved (see [F2], §4 and §8.2). Later A. Avila and M. Viana [AV] proved the simplicity of the Lyapunov spectrum, that is, that all the exponents are non-zero and distinct. The simplicity of the top exponent, that is, the strict inequality $\lambda_{1}^{\mu}>\lambda_{2}^{\mu}$ is much simpler. W. Veech [V] proved it for the canonical absolutely continuous invariant measures on connected components of strata. This result was generalized by the first author in [F2] to an arbitrary Teichmüller invariant ergodic probability measure. 
Recently, Forni has developed his method from [F2] to give a general criterion for the non-uniform hyperbolicity of the Kontsevich-Zorich spectrum for a wide class of $\operatorname{SL}(2, \mathbb{R})$-invariant measures [[F4]. The criterion is based on a topological condition on completely periodic directional foliations contained in the support of the measure.

Closing the considerations of this section, we observe that the following fundamental question is wide open:

Problem 1. Does there exist any finite $\mathrm{SL}(2, \mathbb{R})$-invariant ergodic measure such that the number of strictly positive exponents for the KontsevichZorich cocycle differs from the maximal rank of the bilinear form $B_{\omega}$ at a positive measure set in the space of Abelian differentials?

\section{APPENDIX A. LYAPUNOV SPECTRUM OF SQUARE-TILED CYCLIC COVERS}

Arithmetic Teichmüller curves of square-tiled cyclic covers (see e.g. the subsection below and/or [FMZ] for definitions) provide a basic model for the discussion of relations between the Lyapunov spectrum and the geometry of the Hodge bundle. In particular, we will see that, in the case of square-tiled cyclic covers, the annihilator $\operatorname{Ann}\left(B^{\mathbb{R}}\right)$ of the second fundamental form $B^{\mathbb{R}}$ coincides with the neutral Oseledets bundle $E_{(0)}^{\mu}$. Therefore, in the context of square-tiled cyclic covers, the rank of the second fundamental form (at the generic point with respect to an $\mathrm{SL}(2, \mathbb{R})$-invariant measure) equals the number of strictly positive Lyapunov exponents, and the corank of the second fundamental form is always completely explained by the symmetries of the underlying surfaces.

However, we advance that these relations are not true in general, as an example in Appendix B below will show: actually, the best general results available (on the relationship between $\operatorname{Ann}\left(B^{\mathbb{R}}\right)$ and $\left.E_{(0)}^{\mu}\right)$ were already given above in Theorem 3 , Theorem 4 and Theorem 2 .

A.1. Square-tiled cyclic covers. In the sequel, we recall the definition of square-tiled cyclic covers and some of its basic properties. For more details and proofs of the statements below, see e.g. [FMZ].

Let $N>1$ be an integer, and consider $\left(a_{1}, \ldots, a_{4}\right)$ be a 4 -tuple of integers satisfying the following conditions:

(A.1) $0<a_{i} \leq N ; \quad \operatorname{gcd}\left(N, a_{1}, \ldots, a_{4}\right)=1 ; \quad \sum_{i=1}^{4} a_{i} \equiv 0(\bmod N)$. 
Let $z_{1}, z_{2}, z_{3}, z_{4} \in \mathbb{C}$ be four distinct points. Conditions A.1 imply that, possibly after a desigularization, a Riemann surface defined by equation

$$
w^{N}=\left(z-z_{1}\right)^{a_{1}}\left(z-z_{2}\right)^{a_{2}}\left(z-z_{3}\right)^{a_{3}}\left(z-z_{4}\right)^{a_{4}}
$$

is closed, connected and nonsingular. We denote this Riemann surface by $M=M_{N}\left(a_{1}, a_{2}, a_{3}, a_{4}\right)$. Puncturing the ramification points we obtain a regular $N$-fold cover over $\mathbb{P}^{1}(\mathbb{C}) \backslash\left\{z_{1}, z_{2}, z_{3}, z_{4}\right\}$. The group of deck transformations of this cover is the cyclic group $\mathbb{Z} / N \mathbb{Z}$ with a generator $T: M \rightarrow M$ given by

$$
T(z, w):=(z, \zeta w),
$$

where $\zeta$ is a primitive $N$ th root of unity, $\zeta^{N}=1$.

Next, we recall that any meromorphic quadratic differential $q(z)(d z)^{2}$ with at most simple poles on a Riemann surface defines a flat metric $g(z)=$ $|q(z)|$ with conical singularities at zeroes and poles of $q$. Consider a meromorphic quadratic differential

$$
q_{0}=\frac{(d z)^{2}}{\left(z-z_{1}\right)\left(z-z_{2}\right)\left(z-z_{3}\right)\left(z-z_{4}\right)}
$$

on $\mathbb{P}^{1}(\mathbb{C})$. For a convenient choice of parameters $z_{1}, \ldots, z_{4}$ the rectangles become unit squares. Therefore, given $M_{N}\left(a_{1}, a_{2}, a_{3}, a_{4}\right)$ as above and denoting by $p: M_{N}\left(a_{1}, a_{2}, a_{3}, a_{4}\right) \rightarrow \mathbb{P}^{1}(\mathbb{C})$ the canonical projection $p(z, w)=z$, we have that the quadratic differential $q=p^{*} q_{0}$ on $M_{N}\left(a_{1}, a_{2}, a_{3}, a_{4}\right)$ induces a flat structure naturally tiled by unit squares (by construction). In other words, we get in this way a square-tiled surface (or origami or arithmetic translation surface) $\left(M_{N}\left(a_{1}, \ldots, a_{4}\right), q=p^{*} q_{0}\right)$.

During the present discussion, we will focus exclusively on the orientable case $q=\omega^{2}$ for an Abelian differential $\omega$. By Lemma 2 of [FMZ], this amounts to assume that $N$ is even, and $a_{i}$ is $o d d, i=1,2,3,4$ in what follows. Finally, closing this preliminary subsection on square-tiled cyclic covers, we recall (for later use) the following property:

Lemma A.1 (Lemma 5 of [[FMZ]). In the case $q:=p^{*} q_{0}=\omega^{2}$ for an Abelian differential $\omega$, one has that the form $\omega$ is anti-invariant with respect to the action of a generator of the group of deck transformations,

$$
T^{*} \omega=-\omega .
$$

A.2. Maximally degenerate spectrum in genus four. Before entering into the discussion of the relation between the annihilator $\operatorname{Ann}\left(B^{\mathbb{R}}\right)$ of the second fundamental form and the neutral Oseledets bundle $E_{0}^{\mu}$ in the context of square-tiled cyclic covers, we recall below one of the only two examples of Teichmüller curves of square-tiled cyclic covers with maximally 
degenerate Kontsevich-Zorich spectrum. The first example to be discovered, in genus three, is the Teichmüller curve of the well-known Eierlegende

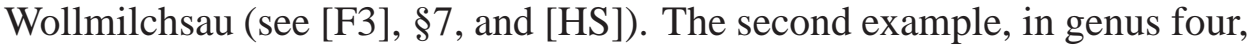
was announced in [FMt $]$ and will be presented below.

These examples were the motivation for a full investigation of the spectrum of square-tiled cyclic covers carried out in [EKZ2] and, from a slightly different perspective, in the next subsections of this appendix. In [FMZ] we showed that there are no other (maximally degenerate) examples among square-tiled cyclic covers (see [Mo], for a stronger result in the class of all Veech surfaces and [Au] for progress on the general case). Both examples were discovered as an application of the symmetry criterion given by Theorem 2 to the arithmetic Teichmüller curves of the square-tiled cyclic covers $M_{4}(1,1,1)$ and $M_{6}(1,1,1,3)$ respectively.

Below, we will follow the presentation in [FMt] and apply the symmetry criterion to the arithmetic Teichmüller curve of the square-tiled cyclic cover $M_{6}(1,1,1,3)$ to derive that it is maximally degenerate.

Corollary A.1. The Lyapunov spectrum of the Hodge bundle over the geodesic flow on the Teichmüller curve of cyclic covers $M_{6}(1,1,1,3)$ is maximally degenerate, $\lambda_{2}=\lambda_{3}=\lambda_{4}=0$.

Proof. By Lemma A.1 the generator $T$ of the group of deck transformations of $M_{6}(1,1,1,3)$ acts on $\omega$ as $T^{*} \omega=-\omega$, see (A.4); in particular $u(T)=-1$, and $u^{2}(T)=1$. We have an explicit basis

$$
\frac{\left(z-z_{4}\right)^{2} d z}{w^{5}}, \frac{\left(z-z_{4}\right)^{3} d z}{w^{5}}, \frac{\left(z-z_{4}\right) d z}{w^{4}} \text { and } \omega:=\frac{\left(z-z_{4}\right) d z}{w^{3}}
$$

in the space of holomorphic 1 -forms on $M_{6}(1,1,1,3)$; the corresponding eigenvalues are

$$
\left\{u_{1}(T), u_{2}(T), u_{3}(T), u_{4}(T)\right\}=\left\{\zeta, \zeta, \zeta^{2}, \zeta^{3}\right\} \quad \text { where } \zeta=\sqrt[3]{-1} .
$$

It is easy to see that for all couples of indices $1 \leq i \leq j \leq 4$ except $(i, j)=(4,4)$ one has $u_{i}(T) u_{j}(T) \neq 1=u^{2}(T)$. By Theorem 2 , the proof is complete.

In both cases of $M_{4}(1,1,1,1)$ and $M_{6}(1,1,1,3)$, the symmetries given by the deck transformations force the rank of the second fundamental form to be equal to 1 (its lowest possible value) and there is exactly 1 strictly positive exponent, the top exponent of the tautological bundle. In the sequel, we will extend this picture to the whole class of square-tiled cyclic covers.

\section{A.3. Rank of the second fundamental form and positive exponents.} The identity between the rank of the second fundamental form and the number of strictly positive Kontsevich-Zorich exponents always holds for square tiled-cyclic covers. In fact, we have the following result. 
Theorem 5. Let $M_{N}\left(a_{1}, a_{2}, a_{3}, a_{4}\right)$ be a square-tiled cyclic cover with $N$ even and all $a_{i}$ odd. The rank of $B_{\omega}$ is constant for all $\omega$ in the corresponding $\mathrm{SL}(2, \mathbb{R})$-orbit and it is equal to the number of strictly positive Kontsevich-Zorich exponents on the Hodge bundle $H_{\mathbb{R}}^{1}$.

The result was inspired by [EKZ2], and it can be obtained as a corollary of the results of that paper. For the sake of completeness, we present in the remaining part of this appendix a proof of Theorem 5. Such a proof is based on Theorem 3 and a remarkable property of square-tiled cyclic covers, namely, the existence of an explicit $\mathrm{SL}(2, \mathbb{R})$-invariant, $B^{\mathbb{R}}$-orthogonal splitting of the Hodge bundle $H_{\mathbb{R}}^{1}$ over a Teichmüller curve of a square-tiled cyclic cover into subbundles of small dimension (2 or 4$)$. We start with the description of the splitting, see [BMo] and [EKZ2] for more details.

Consider a generator $T$ of the group of deck transformations of the cyclic cover, see A.2. The induced linear map

$$
T^{*}: H^{1}(S, \mathbb{C}) \rightarrow H^{1}(S, \mathbb{C})
$$

verifies $\left(T^{*}\right)^{N}=\mathrm{Id}$, hence its eigenvalues are $N$-th roots of unity, that is, they form a subset of the set $\left\{1, \zeta, \ldots, \zeta^{N-1}\right\}$, where $\zeta$ is an $N$-th primitive root of unity. For all $k \in\{1, \ldots, N-1\}$, let

$$
V_{k}:=\operatorname{Ker}\left(T^{*}-\zeta^{k} \mathrm{Id}\right) \subset H^{1}(S, \mathbb{C}) .
$$

Since the deck transformation $T$ commutes with the $\operatorname{SL}(2, \mathbb{R})$-action, each $V_{k}$ is $\mathrm{SL}(2, \mathbb{R})$-invariant subbundle of $H_{\mathbb{C}}^{1}$.

On the other hand, $T^{*}$ has a well-defined restriction to $H^{1,0}(S, \mathbb{C})=$ $H^{1,0}$, and $\left(\left.T^{*}\right|_{H^{1,0}(S, \mathbb{C})}\right)^{N}=\mathrm{Id}$, so that we can also define

$$
V_{k}^{1,0}:=\operatorname{Ker}\left(T^{*}-\zeta^{k} \mathrm{Id}\right) \subset H^{1,0}(S, \mathbb{C}) .
$$

In general, the subspaces $V_{k}^{1,0}$ do not form $\mathrm{SL}(2, \mathbb{R})$-invariant subbundles of the complex cohomology bundle.

Let us consider the decomposition

$$
H^{1}(S, \mathbb{C})=H^{1,0}(S) \oplus H^{0,1}(S)
$$

Since the operator $T^{*}$ preserves the subspace $H^{1,0}(S)$ and commutes with complex conjugation, it follows that

$$
H^{1}(S, \mathbb{C})=\bigoplus_{k=1}^{N-1} V_{k}=\bigoplus_{k=1}^{N-1}\left(V_{k}^{1,0} \oplus V_{k}^{0,1}\right)=\bigoplus_{k=1}^{N-1}\left(V_{k}^{1,0} \oplus \overline{V_{N-k}^{1,0}}\right)
$$

In particular, by defining

$$
W_{k}:=\left\{\begin{array}{ll}
V_{k} \oplus V_{N-k} & \text { for } k=1, \ldots, \frac{N}{2}-1 \\
V_{N / 2} & \text { for } k=\frac{N}{2}
\end{array} .\right.
$$


and

$$
H_{k}^{1}(S, \mathbb{R}):=W_{k} \cap H^{1}(S, \mathbb{R}),
$$

we see that, for any $k<N / 2$, one has

$$
W_{k}=\left(V_{k}^{1,0} \oplus V_{N-k}^{1,0}\right) \oplus\left(\overline{V_{k}^{1,0} \oplus V_{N-k}^{1,0}}\right)
$$

and, for $k=N / 2$, one gets

$$
W_{N / 2}=V_{N / 2}^{1,0} \oplus \overline{V_{N / 2}^{1,0}}
$$

Thus, each $H_{k}^{1}(S, \mathbb{R})$ is $\operatorname{SL}(2, \mathbb{R})$-invariant and Hodge star-invariant.

Concerning the dimensions of these subbundles, we have the following lemma. Denote

$$
t(k):=\sum_{i=1}^{4}\left\{\frac{k a_{i}}{N}\right\}, \quad \text { for } k=1, \ldots, N-1,
$$

where $\{x\}$ denotes a fractional part of $x$. Conditions A.1 imply that $t(k)$ is integer, hence, clearly, $t(k) \in\{1,2,3\}$.

Lemma $([\bar{B}]$, Lemma 4.3). For any $k \in\{1, \ldots, N-1\}$, one has

$$
\operatorname{dim}_{\mathbb{C}} V_{k}^{1,0}=t(N-k)-1 \in\{0,1,2\} .
$$

Define the following two complementary subsets $\mathcal{J}_{0}$ and $\mathcal{J}_{1}$ of the set $\{1, \ldots, N / 2\}$ :

$$
\begin{gathered}
\mathcal{J}_{0}:=\left\{k \mid 1 \leq k \leq N / 2, \text { and at least one of } V_{k}^{1,0} \text { or } V_{N-k}^{1,0} \text { vanish }\right\}, \\
\mathcal{J}_{1}:=\{1, \ldots, N / 2\} \backslash \mathcal{J}_{0},
\end{gathered}
$$

and consider the subspaces

$$
H_{\mathcal{J}_{0}}^{1}(S, \mathbb{R}):=\bigoplus_{k \in \mathcal{J}_{0}} H_{k}^{1}(S, \mathbb{R}), \quad H_{\mathcal{J}_{1}}^{1}(S, \mathbb{R}):=\bigoplus_{k \in \mathcal{J}_{1}} H_{k}^{1}(S, \mathbb{R})
$$

of the real cohomology. By definition,

$$
H^{1}(S, \mathbb{R})=H_{\mathcal{J}_{0}}^{1}(S, \mathbb{R}) \oplus H_{\mathcal{J}_{1}}^{1}(S, \mathbb{R}) .
$$

In this language, a consequence of the previous lemma is:

Corollary A.2. An integer $k$, such that $1 \leq k \leq N-1$, belongs to the subset $\mathcal{J}_{1}$ if and only if

$$
\operatorname{dim} V_{k}^{1,0}=\operatorname{dim} V_{N-k}^{1,0}=1 .
$$


Proof. The definition A.7) of the integers $t(k)$ and conditions (A.1) on the sum of $a_{i}$ imply that $t(k)+t(N-k) \in\{2,3,4\}$, or, equivalently,

$$
\operatorname{dim} V_{k}^{1,0}+\operatorname{dim} V_{N-k}^{1,0} \in\{0,1,2\} .
$$

It follows that if $k \in \mathcal{J}_{1}$ then the integers $\operatorname{dim} V_{k}^{1,0}$ and $\operatorname{dim} V_{k}^{1,0}$ are both different from zero, hence they have to be both equal to 1 , as claimed.

In any case, for a square-tiled cyclic cover, we can use these subbundles to compute the complex bilinear form $B$ on $H^{1,0}$ as follows.

Lemma A.2. Let $\omega_{j}, \omega_{k}$ be eigenvectors of the linear map $\left.T^{*}\right|_{H^{1,0}(S, \mathbb{C})}$ with eigenvalues $\zeta^{j}$ and $\zeta^{k}$ respectively. The following formula holds:

$$
B_{\omega}\left(\omega_{j}, \omega_{k}\right) \begin{cases}\neq 0 & \text { if } j=N-k \\ =0 & \text { otherwise. }\end{cases}
$$

Proof. Symmetry arguments analogous to the ones in the proof of Theorem 2 show that, in our case, if the eigenvalues $u_{j}=\zeta^{j}$ and $u_{k}=\zeta^{k}$ are not complex-conjugate, the value $B_{\omega}\left(\omega_{j}, \omega_{k}\right)$ on the corresponding eigenvectors is equal to zero. Since $\zeta^{k}=\overline{\zeta^{N-k}}=1 / \zeta^{N-k}$, one has that $j \neq N-k$ implies $B_{\omega}\left(\omega_{j}, \omega_{k}\right)=0$.

Now, consider the action of $T^{*}$ on the Abelian differential $\left(\omega_{k} \cdot \omega_{N-k}\right) / \omega$. By Lemma A.1, we get

$$
T^{*}\left(\frac{\omega_{k} \omega_{N-k}}{\omega}\right)=\frac{\zeta^{k} \omega_{k} \cdot \zeta^{N-k} \omega_{N-k}}{(-\omega)}=-\omega_{k} \omega_{N-k},
$$

that is, $\left(\omega_{k} \cdot \omega_{N-k}\right) / \omega$ is $T^{*}$ anti-invariant. In other words, since $\zeta^{N / 2}=-1$, we have that $\left(\omega_{k} \cdot \omega_{N-k}\right) / \omega \in V^{1,0}(N / 2)$. By Corollary A.2, this implies that $\left(\omega_{k} \cdot \omega_{N-k}\right)$ is proportional to $\omega$ with a nonzero constant coefficient const. Thus,

$$
B_{\omega}\left(\omega_{k}, \omega_{N-k}\right):=\frac{i}{2} \int_{S} \frac{\omega_{k} \omega_{N-k}}{\omega} \bar{\omega}=\text { const } \cdot \frac{i}{2} \int_{S} \omega \bar{\omega}=\text { const } \cdot 1 \neq 0
$$

This completes the proof of the lemma.

Our discussion so far can be summarized by the following lemma:

Lemma A.3. The real Hodge bundle $H_{\mathbb{R}}^{1}$ over an arithmetic Teichmüller curve of a square-tiled cyclic cover splits into a direct sum

$$
H^{1}(S, \mathbb{R})=\bigoplus_{k=1}^{N / 2} H_{k}^{1}(S, \mathbb{R})
$$

of $\mathrm{SL}(2, \mathbb{R})$-invariant, $B^{\mathbb{R}}$-orthogonal, Hodge star-invariant subbundles. 
Remark A.1. By Lemma 3.4 and Lemma A.3 the subspaces $H_{k}^{1}(S, \mathbb{R})$ in the splitting (A.8) are symplectic-orthogonal and Hodge-orthogonal. Of course, it can be also immediately seen directly.

Given a Teichmüller curve $\mathcal{C}$ associated to a square-tiled cyclic cover, we denote $H_{\mathcal{J}_{0}}^{1} \subset H_{\mathbb{R}}^{1}$ the bundle over $\mathcal{C}$ formed by the subspaces $H_{\mathcal{J}_{0}}^{1}(S, \mathbb{R})$, and $E_{0} \subset H_{\mathbb{R}}^{1}$ be the Oseledets bundle corresponding to the zero Lyapunov exponents (with respect to the unique $\mathrm{SL}(2, \mathbb{R})$-invariant probability supported on $\mathcal{C}$ ). The Lemma below is a consequence of Theorem 3 and Lemma A.2

Lemma A.4. The following inclusions hold:

$$
H_{\mathcal{J}_{0}}^{1} \subseteq \operatorname{Ann}\left(B^{\mathbb{R}}\right) \quad \text { and } \quad H_{\mathcal{J}_{0}}^{1} \subseteq E_{0} .
$$

Proof. For each $k \in \mathcal{J}_{0}$, one has $V_{k}^{1,0} \oplus V_{N-k}^{1,0}=V_{N-k}^{1,0}$ or $V_{k}^{1,0}$. Hence $\left(\left.B\right|_{V_{k}^{1,0} \oplus V_{N-k}^{1,0}}\right)=0$ by Lemma A.2. It follows that $\left.B^{\mathbb{R}}\right|_{H_{J_{0}}^{1}}=0$, that is, $H_{\mathcal{J}_{0}}^{1} \subseteq \operatorname{Ann}\left(B^{\mathbb{R}}\right)$. On the other hand, we have that, by construction, $H_{\mathcal{J}_{0}}^{1}$ is $\mathrm{SL}(2, \mathbb{R})$-invariant and Hodge star-invariant. Thus, the inclusion $H_{\mathcal{J}_{0}}^{1} \subseteq E_{0}$ follows from Theorem 3 (and the inclusion $H_{\mathcal{J}_{0}}^{1} \subseteq \operatorname{Ann}\left(B^{\mathbb{R}}\right)$ ).

In what follows, we want to show that the inclusions in the previous lemma are actually equalities. The first step is to prove the following fact:

Lemma A.5. The following identity holds:

$$
H_{\mathcal{J}_{0}}^{1}=\operatorname{Ann}\left(B^{\mathbb{R}}\right) \text {. }
$$

Proof. For any $k \in \mathcal{J}_{1}$, take $\omega_{k} \in V_{k}^{1,0}-\{0\}$ and $\omega_{N-k} \in V_{N-k}^{1,0}-\{0\}$. It follows from Corollary A.2 that such $\omega_{k}$ and $\omega_{N-k}$ exist, and $V_{k}^{1,0}=\mathbb{C} \omega_{k}$, $V_{N-k}^{1,0}=\mathbb{C} \omega_{N-k}$. By A.5 and A.6, we obtain that $\operatorname{Re} \omega_{k}, \operatorname{Re} \omega_{N-k}$, $\operatorname{Im} \omega_{k}, \operatorname{Im} \omega_{N-k}$ is a basis of $H_{k}^{1}(S, \mathbb{R})$ when $k \neq N / 2$, and $\operatorname{Re} \omega_{N / 2}$, $\operatorname{Im} \omega_{N / 2}$ is a basis of $H_{N / 2}^{1}(S, \mathbb{R})$. By Lemma A.2 we deduce that the $\left.B^{\mathbb{R}}\right|_{H_{k}^{1}(S, \mathbb{R})}$ is non-degenerate for any $k \in \mathcal{J}_{1}$. Since $H_{\mathcal{J}_{1}}^{1}(S, \mathbb{R})$ is a $B^{\mathbb{R}}$ orthogonal sum (see Lemma A.3), it follows that the restriction of $B^{\mathbb{R}}$ to $H_{\mathcal{J}_{1}}^{1}(S, \mathbb{R})$ is non-degenerate. Finally, since $H^{1}(S, \mathbb{R})$ splits as a $B^{\mathbb{R}}$ orthogonal sum of $H_{\mathcal{J}_{1}}^{1}(S, \mathbb{R})$ and $H_{\mathcal{J}_{0}}^{1}(S, \mathbb{R})$ (see again Lemma A.3), it follows that $\operatorname{Ann}\left(B^{\mathbb{R}}\right) \subseteq H_{\mathfrak{J}_{0}}^{1}$. Because the converse inclusion was proved as part of Lemma A.4 the proof of this lemma is complete.

Next, we invoke the following key result:

Theorem 6 (Theorem 2.6, item (iii), of [EKZ2]). For every $k \in \mathcal{J}_{1}$ the Kontsevich-Zorich cocycle has no zero exponents on the $\mathrm{SL}(2, \mathbb{R})$-invariant subbundle $H_{k}^{1} \subset H_{\mathbb{R}}^{1}$. Moreover, for each $k \in \mathcal{J}_{1}-\{N / 2\}$, the Lyapunov spectrum of Kontsevich-Zorich cocycle restricted to $H_{k}^{1}$ has the form 
$\left\{\lambda_{k}, \lambda_{k},-\lambda_{k},-\lambda_{k}\right\}$ with $\lambda_{k}>0$ (i.e., there is only one double positive Lyapunov exponent).

Remark A.2. This theorem could be deduced directly from the properties of $\left.B^{\mathbb{R}}\right|_{H_{k}^{1}}$ discussed in this paper. However, we prefer to skip the presentation of this proof because, contrary to the arguments in [EKZ2], it doesn't yield the precise value $\lambda_{k}=2 \cdot \min \left\{t_{j}(k), 1-t_{j}(k): j=1,2,3,4\right\}$.

At this stage, we are ready to get the following result stating in particular that for square-tiled cyclic covers the central Oseledets subbundle indeed coincides with the kernel of the second fundamental form:

Theorem 7. Suppose that $N$ is even and all $a_{i}$ are odd. Consider the $\mathrm{SL}(2, \mathbb{R})$-orbit of the square-tiled cyclic cover $M_{N}\left(a_{1}, a_{2}, a_{3}, a_{4}\right)$. The following identities hold:

$$
E_{0}=\operatorname{Ann}\left(B^{\mathbb{R}}\right)=H_{\mathcal{J}_{0}}^{1} .
$$

In particular the number of strictly positive Lyapunov exponents of the Kontsevich-Zorich cocycle is given by the following formula:

$$
\#\left\{k \in\{1, \ldots, N-1\} \mid \operatorname{dim}_{\mathbb{C}} V_{k}^{1,0}=\operatorname{dim}_{\mathbb{C}} V_{N-k}^{1,0}=1\right\} .
$$

Proof. The decomposition A.8 of the Hodge bundle into a direct sum of $\mathrm{SL}(2, \mathbb{R})$-invariant subbundles implies that the Lyapunov spectrum of the Hodge bundle is the union of the spectra of the subbundles. Lemmas A.4 and $\mathrm{A} .5$ show that $\operatorname{Ann}\left(B^{\mathbb{R}}\right)=H_{\mathcal{J}_{0}}^{1} \subseteq E_{0}$, while Theorem 6 , shows that the inclusion $H_{\mathcal{J}_{0}}^{1} \subseteq E_{0}$ is, actually an equality. Finally, the formula counting the number of nonnegative Lyapunov exponents is obtained by a combination of Theorem 6 with Corollary A.2.

Theorem 5 is now an immediate corollary of the more precise Theorem 7

APPENDIX B. LYAPUNOV SPECTRUM OF A HIGHER-DIMENSIONAL

$$
\operatorname{SL}(2, \mathbb{R}) \text {-INVARIANT LOCUS } z
$$

This appendix is devoted to the description of an $\mathrm{SL}(2, \mathbb{R})$-invariant locus $z \subset \mathcal{H}\left(8,2^{5}\right)$ supporting an ergodic $\operatorname{SL}(2, \mathbb{R})$-invariant probability $\mu$ with the following properties:

- there are precisely 6 vanishing exponents out of 10 non-negative Kontsevich-Zorich exponents (with respect to $\mu$ ),

- the corank of $B_{\omega}$ is 6 for every $\omega \in \mathcal{Z}$, but

- $E_{(0)}^{\mu} \neq \operatorname{Ann}\left(B^{\mathbb{R}}\right), E_{(0)}^{\mu}$ is not $\operatorname{SL}(2, \mathbb{R})$-invariant and $\operatorname{Ann}\left(B^{\mathbb{R}}\right)$ is not flow-invariant.

The construction of $z$ is partly motivated by McMullen's paper [Mc].

Below, we will only sketch the proof of these properties. The details are part of a forthcoming paper [FMZ2]. 
B.1. Description of the locus $z$. Denote by $z$ the family of Riemann surfaces

$$
C_{6}=\left\{w^{6}=\left(z-z_{1}\right) \ldots\left(z-z_{6}\right)\right\}
$$

equipped with the Abelian differentials

$$
\omega=\frac{\left(z-z_{1}\right) d z}{w^{3}} .
$$

Here, $z_{1}, \ldots, z_{6} \in \mathbb{P}^{1}(\mathbb{C})$ are 6 pairwise distinct points of the Riemann sphere. A quick inspection reveals that these Riemann surfaces have genus 10 , and any such $\omega$ has a zero of order 8 at the branch point over $z_{1}$ and a double zero at the branch point over $z_{j}, j=2, \ldots, 6$. In other words, $z \subset \mathcal{H}\left(8,2^{5}\right)$.

Observe that $\omega^{2}=g^{*} q$ where $g: C_{6} \rightarrow \mathbb{P}^{1}(\mathbb{C}), g(z, w)=z$, and $q$ is the following quadratic differential with a simple zero and 5 simple poles of the Riemann sphere:

$$
q=\frac{\left(z-z_{1}\right) d z^{2}}{\left(z-z_{2}\right) \ldots\left(z-z_{6}\right)} \in \mathcal{Q}\left(1,-1^{5}\right)
$$

Alternatively, $\omega=h^{*} \widehat{\omega}$ where $h: C_{6} \rightarrow C_{2}, h(z, w)=\left(z, w^{3}\right), C_{2}=$ $\left\{y^{2}=\left(z-z_{1}\right) \ldots\left(z-z_{6}\right)\right\}$ is a genus two Riemann surface and $\widehat{\omega}$ is the following Abelian differential on $C_{2}$ :

$$
\widehat{\omega}=\frac{\left(z-z_{1}\right) d z}{y} \in \mathcal{H}(2) .
$$

Since $Z, Q\left(1,-1^{5}\right)$ and $\mathcal{H}(2)$ are 4-dimensional loci, it follows that $Z \simeq$ $\mathcal{Q}\left(1,-1^{5}\right) \simeq \mathcal{H}(2)$ and $\mathcal{Z}$ is the closure of the $\mathrm{GL}^{+}(2, \mathbb{R})$-orbit of $\left(C_{6}, \omega\right)$ for a generic choice of $z_{1}, \ldots, z_{6}$.

B.2. Decomposition of Hodge bundle over z. Similarly to the discussion of the case of square-tiled cyclic covers in Appendix A above, we notice that the group of deck transformations of the cover $g: C_{6} \rightarrow \mathbb{P}^{1}(\mathbb{C})$ (ramified over $z_{1}, \ldots, z_{6}$ ) is generated by

$$
T(z, w)=(z, \varepsilon w)
$$

where $\varepsilon$ is a primitive 6 th root of unity. For sake of concreteness, we take $\varepsilon=\exp (2 \pi i / 6)$. Again, we denote by $T^{*}: H^{1}\left(C_{6}, \mathbb{C}\right) \rightarrow H^{1}\left(C_{6}, \mathbb{C}\right)$ the induced linear map. Of course, since $\left(T^{*}\right)^{6}=\mathrm{Id}$, its eigenvalues are a subset of $\left\{1, \varepsilon, \ldots, \varepsilon^{5}\right\}$. For every $k=1, \ldots, 5$, we put

$$
V_{k}=\operatorname{Ker}\left(T^{*}-\varepsilon^{k} \mathrm{Id}\right) \text {. }
$$

Again, because $\mathrm{SL}(2, \mathbb{R})$ acts by monodromy of the flat Gauss-Manin connection, these eigenspaces $V_{k}$ form $\mathrm{SL}(2, \mathbb{R})$-invariant subbundles of the Hodge bundle over $z$. 
Next, we consider the restriction of $T^{*}$ to $H^{1,0}\left(C_{6}, \mathbb{C}\right)$. Because there is no Abelian differential on $\mathbb{P}^{1}(\mathbb{C})$, we see that the eigenvalues of $\left.T^{*}\right|_{H^{1,0}\left(C_{6}, \mathbb{C}\right)}$ form a subset of $\left\{\varepsilon^{j}: j=1, \ldots, 5\right\}$ and we denote

$$
V_{k}^{1,0}=\operatorname{Ker}\left(\left.T^{*}\right|_{H^{1,0}\left(C_{6}, \mathbb{C}\right)}-\varepsilon^{k} \mathrm{Id}\right),
$$

so that

$$
H^{1,0}\left(C_{6}, C\right)=\bigoplus_{k=1}^{5} V_{k}^{1,0} .
$$

A quick computation shows that

$$
\left\{\frac{z^{j} d z}{w^{k}}: 0<j<k<5\right\}
$$

is a basis of holomorphic differentials on the genus 10 Riemann surface $C_{6}$. In particular,

$$
\operatorname{dim}_{\mathbb{C}} V_{k}^{1,0}=k-1
$$

for each $k=1, \ldots, 5$.

Finally, we form the subspaces

$$
W_{k}=\left\{\begin{array}{cc}
V_{k} \oplus V_{6-k} & \text { if } k \neq 3 \\
V_{3} & \text { if } k=3
\end{array}\right.
$$

and

$$
H_{k}^{1}\left(C_{6}, \mathbb{R}\right):=W_{k} \cap H^{1}\left(C_{6}, \mathbb{R}\right) .
$$

Since $V_{k} \oplus V_{6-k}$ and $V_{3}$ are invariant under complex conjugation, each $V_{k}$ is $\operatorname{SL}(2, \mathbb{R})$-invariant and $V_{k}=V_{k}^{1,0} \oplus V_{k}^{0,1}=V_{k}^{1,0} \oplus \overline{V_{6-k}^{1,0}}$, we have the following Hodge- $*$ and $\mathrm{SL}(2, \mathbb{R})$-invariant splitting

$$
H^{1}\left(C_{6}, \mathbb{R}\right)=\bigoplus_{k=1}^{3} H_{k}^{1}\left(C_{6}, \mathbb{R}\right) .
$$

Moreover, a direct application of the arguments of the proof of Theorem 2 shows that the $H_{k}^{1}\left(C_{6}, \mathbb{R}\right)$ are pairwise $B^{\mathbb{R}}$-orthogonal.

In the sequel, we will use these decompositions to analyze $\operatorname{Ann}\left(B^{\mathbb{R}}\right)$ and the neutral Oseledets bundle of $\mathrm{SL}(2, \mathbb{R})$-invariant ergodic probabilities supported on $z$.

B.3. Neutral Oseledets bundle versus $\operatorname{Ann}\left(B^{\mathbb{R}}\right)$ over $Z_{\text {. In the present }}$ case, we just saw that $\operatorname{dim}_{\mathbb{C}} V_{k}^{1,0}=k-1$, so that $V_{1}=\overline{V_{5}^{1,0}}, V_{5}=V_{5}^{1,0}$ and, a fortiori, $V_{5}^{1,0} \subset \operatorname{Ann}(B)$ and $H_{1}^{1}\left(C_{6}, \mathbb{R}\right) \subset \operatorname{Ann}\left(B^{\mathbb{R}}\right)$ (compare with Theorem 2 and Lemma A.2). Furthermore, from Theorem 3 , it follows also that $H_{1}^{1}\left(C_{6}, \mathbb{R}\right) \subset E_{(0)}^{\mu}$ for any ergodic $\mathrm{SL}(2, \mathbb{R})$-invariant $\mu$ supported on $z$, 
that is, any such measure automatically possesses at least 4 vanishing exponents among the 10 non-negative exponents of Kontsevich-Zorich cocycle restricted to the Hodge bundle over $z$.

Remark B.1. The fact $V_{5}^{1,0}=V_{5}$ says that the Jacobian of $C_{6}$ has a fixed part (of dimension $4=\operatorname{dim}_{\mathbb{C}} V_{5}$ ), i.e., the complex torus $A=V_{5}^{1,0} / V_{5}^{1,0}(\mathbb{Z})$ obtained from the quotient of $V_{5}^{1,0}$ by the lattice $V_{5}^{1,0}(\mathbb{Z})=V_{5}^{1,0} \cap H^{1}\left(C_{6}, \mathbb{Z}\right)$ is a fixed part (rigid factor) of the Jacobian $\operatorname{Jac}\left(C_{6}\right)$ of $C_{6}$ in the sense that we have a isogeny $\operatorname{Jac}\left(C_{6}\right) \rightarrow J\left(C_{6}\right) \times A$. The fact that $V_{5}^{1,0}$ is a fixed part of the Jacobian was already known by C. McMullen [Mc, Theorem 8.3] and it was our starting point to study the locus $z$.

Next, we pass to the analysis of $H_{3}^{1}\left(C_{6}, \mathbb{R}\right)$. Because $h^{*}\left(H^{1,0}\left(C_{2}, \mathbb{C}\right)\right)$ equals $V_{3}^{1,0}$ (where $h: C_{6} \rightarrow C_{2}, h(z, w)=\left(z, w^{3}\right)$ is the covering map used above to construct the isomorphism $z \simeq \mathcal{H}(2)$ ), by the results of Bainbridge [Ba] and Eskin, Kontsevich and Zorich [EKZ1], we conclude that the non-negative exponents of the Kontsevich-Zorich cocycle restricted to $H_{3}^{1}\left(C_{6}, \mathbb{R}\right)$ are 1 and $1 / 3$ for any ergodic $\operatorname{SL}(2, \mathbb{R})$-invariant probability supported on $Z$ and the rank of the second fundamental form $B$ restricted to $V_{3}^{1,0}$ has full rank (equal to 2 ).

Therefore, it remains to study the restriction of the second fundamental form $B$ to $W_{2}^{1,0}:=V_{2}^{1,0} \oplus V_{4}^{1,0}$ and the restriction of the Kontsevich-Zorich cocycle to $H_{2}^{1}\left(C_{6}, \mathbb{R}\right)$.

Remark B.2. For sake of concreteness, we observe that $H_{2}^{1}\left(C_{6}, \mathbb{R}\right)$ is a copy of the first homology group $H^{1}\left(C_{3}, \mathbb{R}\right)$ of the genus 4 Riemann surface

$$
C_{3}:=\left\{x^{3}=\left(z-z_{1}\right) \ldots\left(z-z_{6}\right)\right\}=C_{6} /\left\langle T^{2}\right\rangle
$$

Moreover, the square of the Abelian differential $\omega$ on $C_{6}$ projects into the quadratic differential $q_{(4)}=\left(z-z_{1}\right)^{2} d z^{2} / x^{3}$. In other words, the study of the restriction of the Kontsevich-Zorich cocycle over $z$ to $H_{2}^{1}\left(C_{6}, \mathbb{R}\right)$ is equivalent to the study of the Kontsevich-Zorich cocycle over the locus determined by the family of quadratic differentials $\left(C_{3}, q_{(4)}\right) \in \mathcal{Q}\left(7,1^{5}\right)$ of genus 4. In fact, in the forthcoming paper [FMZ2], we will adopt the latter point of view (i.e., we will study directly this family of quadratic differentials of genus 4).

Lemma B.1. The restriction of the form $B$ to $W_{2}^{1,0}$ has rank 2. In particular, $\operatorname{Ann}\left(B^{\mathbb{R}}\right) \cap H_{2}^{1}\left(C_{6}, \mathbb{R}\right)$ is a 4-dimensional (real) subspace of the 8-dimensional space $H_{2}^{1}\left(C_{6}, \mathbb{R}\right)$.

Proof. Let $\alpha \in V_{2}^{1,0}$ and $\beta_{0}, \beta_{1}, \beta_{2} \in V_{4}^{1,0}$ be a basis of $W_{2}^{1,0}$. By the arguments in the proof of Theorem 2, we have that $B_{\omega}(\alpha, \alpha)=0$ and 
$B_{\omega}\left(\beta_{j}, \beta_{l}\right)=0$ for all $0 \leq j, l \leq 3$. Hence, the matrix of $\left.B\right|_{W_{2}^{1,0}}$ in the basis $\left\{\alpha, \beta_{0}, \beta_{1}, \beta_{2}\right\}$ is

$$
\left(\begin{array}{cccc}
0 & b_{0} & b_{1} & b_{2} \\
b_{0} & 0 & 0 & 0 \\
b_{1} & 0 & 0 & 0 \\
b_{2} & 0 & 0 & 0
\end{array}\right)
$$

where $b_{j}:=B_{\omega}\left(\alpha, \beta_{j}\right), j=0,1,2$. Hence, it suffices to prove that one of the entries $b_{j}$ is non-zero to conclude that the rank of $\left.B\right|_{W_{2}^{1,0}}$ is 2 . To do so, we make the following choice of basis $\alpha=d z / w^{2}, \beta_{j}=\left(z-z_{1}\right)^{j} d z / w^{4}$, and we compute

$$
B_{\omega}\left(\alpha, \beta_{2}\right)=\int \frac{\alpha \beta_{2}}{\omega} \bar{\omega}=\int \frac{\left|z-z_{1}\right|^{2}}{|w|^{6}} d z \overline{d z} \neq 0,
$$

that is $b_{2} \neq 0$.

Lemma B.2. The non-negative part of the Lyapunov spectrum of the restriction of the Kontsevich-Zorich cocycle to $H_{2}^{1}\left(C_{6}, \mathbb{R}\right)$ with respect to any $\mathrm{SL}(2, \mathbb{R})$-invariant ergodic probability $\mu$ has the form

$$
\left\{\lambda^{\mu}=\lambda^{\mu}>0=0\right\} .
$$

In particular, the neutral Oseledets bundle $E_{0}^{\mu}$ intersects $H_{2}^{1}\left(C_{6}, \mathbb{R}\right)$ in a subspace of real dimension 4.

We begin the proof of this lemma by following [EKZ2] to see that the Lyapunov exponents of $\left.G_{t}^{K Z}\right|_{H_{2}^{1}\left(C_{6}, \mathbb{R}\right)}$ have multiplicity 2 (at least). Indeed, given a vector $v \in H_{2}^{1}\left(C_{6}, \mathbb{R}\right)$ corresponding to a Lyapunov exponent $\lambda$, we have that $T^{*} v$ corresponds to the same Lyapunov exponent (as $T^{*}$ commutes with the monodromy). On the hand, since the eigenvalues of $\left.T^{*}\right|_{H_{2}^{1}\left(C_{6}, \mathbb{R}\right)}$ are not real (i.e., $\varepsilon^{2}, \varepsilon^{4} \notin \mathbb{R}$ ), it follows that $T^{*} v$ is not collinear to $v$, that is, $\lambda$ has multiplicity 2 at least.

Consequently, the non-negative part of the Lyapunov spectrum of $G_{t}^{K Z}$ restricted to $H_{2}^{1}\left(C_{6}, \mathbb{R}\right.$ ) (with respect to $\mu$ ) has the form

$$
\left\{\lambda^{\mu}=\lambda^{\mu} \geq \theta^{\mu}=\theta^{\mu}\right\} \text {. }
$$

On the other hand, we know that $B^{\mathbb{R}}$ restricted to $H_{2}^{1}\left(C_{6}, \mathbb{R}\right)$ is not degenerate (see Lemma B.1). Hence, from Theorem 3, we conclude that

$$
\lambda^{\mu}>0 \text {. }
$$

In other words, the proof of the previous lemma is reduced to show that $\theta^{\mu}=0$, that is,

Lemma B.3. The non-negative part of the Lyapunov spectrum of $G_{t}^{K Z}$ restricted to $H_{2}^{1}\left(C_{6}, \mathbb{R}\right)$ has two vanishing exponents. 
In the forthcoming paper [ [FMZ2], we will deduce this lemma along the following lines. Since $V_{4}=V_{4}^{1,0} \oplus \overline{V_{2}^{1,0}}, \operatorname{dim}_{\mathbb{C}}\left(V_{4}^{1,0}\right)=3$, and $\operatorname{dim}_{\mathbb{C}}\left(V_{2}^{1,0}\right)=$ 1 , the intersection form (2.1) has signature $(3,1)$ and hence the action of $\mathrm{SL}(2, \mathbb{R})$ through monodromy of the Gauss-Manin connection on the subspace $V_{4}$ of the complex Hodge bundle $H_{\mathbb{C}}^{1}$ is represented by $U(3,1)$ matrices. In [FMZ2], we will see that, in general, a cocycle preserving a pseudoHermitian form of signature $(p, q)$ (i.e., with values in the matrix group $U(p, q))$ has $|p-q|$ zero Lyapunov exponents at least. By applying this general principle in the context of the previous lemma, we have that the Kontsevich-Zorich cocycle has (at least) $3-1=2$ zero Lyapunov exponents, so that the lemma follows.

In any event, by Lemmas B.1 and B.3, we can discuss the main result of this appendix. In the following theorem, we denote by $\mu$ the natural $\mathrm{SL}(2, \mathbb{R})$-invariant ergodic probability fully supported on $z$ (obtained from the so-called Masur-Veech probability on $\mathcal{H}(2)$ via the isomorphism $z \simeq$ $\mathcal{H}(2)$ previously constructed).

Theorem 8. We have Ann $\left(B^{\mathbb{R}}\right) \cap H_{2}^{1}\left(C_{6}, \mathbb{R}\right) \neq E_{(0)}^{\mu} \cap H_{2}^{1}\left(C_{6}, \mathbb{R}\right)$. Consequently, $\operatorname{Ann}\left(B^{\mathbb{R}}\right) \neq E_{(0)}^{\mu}$, hence $E_{(0)}^{\mu}$ is not $\mathrm{SL}(2, \mathbb{R})$-invariant .

Actually, this theorem is a consequence of the following fact:

Theorem 9. There is no 2-dimensional Teichmüller-flow and $\operatorname{SO}(2, \mathbb{R})$ invariant (i.e., $\operatorname{SL}(2, \mathbb{R})$-invariant ) continuous subbundle $V \subset W_{2}^{1,0}$.

Assuming momentarily this theorem, one can conclude Theorem 8 as follows. We have that $\operatorname{Ann}(B) \cap W_{2}^{1,0}$ is a continuous (actually, real-analytic) and $\operatorname{SO}(2, \mathbb{R})$-invariant 2-dimensional subbundle of $W_{2}^{1,0}$. From Theorem 9 it follows that $\operatorname{Ann}(B) \cap W_{2}^{1,0}$ is not flow-invariant, and hence $\operatorname{Ann}\left(B^{\mathbb{R}}\right) \cap$ $H_{2}^{1}\left(C_{6}, \mathbb{R}\right)$ is not flow-invariant. Since $E_{(0)}^{\mu} \cap H_{2}^{1}\left(C_{6}, \mathbb{R}\right)$ is flow-invariant, it follows that $E_{(0)}^{\mu} \cap H_{2}^{1}\left(C_{6}, \mathbb{R}\right)$ cannot coincide with $\operatorname{Ann}\left(B^{\mathbb{R}}\right) \cap H_{2}^{1}\left(C_{6}, \mathbb{R}\right)$. By Theorem 3 , if $E_{(0)}^{\mu}$ were $\operatorname{SL}(2, \mathbb{R})$-invariant, it would be a subbundle of the bundle $\operatorname{Ann}\left(B^{\mathbb{R}}\right)$, hence it would coincide with it by dimensional reasons, thereby contradicting the first part of the statement.

Concerning the proof of Theorem 9, let us just say a few words (for more details see [FMZ2] $)$ : the basic idea is that $\operatorname{SL}(2, \mathbb{R})$-invariance of a continuous subbundle $V$ can be tested along pseudo-Anosovs (i.e., periodic orbits of Teichmüller flow); indeed, the existence of $\mathrm{SL}(2, \mathbb{R})$-invariant continuous subbundles $V$ implies that the monodromy matrices along pseudo-Anosovs passing by the same Riemann surface should share a common subspace, and (the non-validity of) this last property can be tested by direct calculation.

Closing this appendix, let us mention that the precise value of the positive Lyapunov exponent coming from $H_{2}^{1}\left(C_{6}, \mathbb{R}\right)$ can be computed from the 
main formula of [EKZ1] and a computation with Siegel-Veech constants related to $\mathcal{Q}\left(1,-1^{5}\right)$ (see [FMZ2]):

Proposition 3. Let $\nu$ be any $\mathrm{SL}(2, \mathbb{R})$-invariant ergodic probability supported on $z$. Then, the non-negative part of the Lyapunov spectrum of the Kontsevich-Zorich cocycle with respect to $\nu$ has the form

$$
\{1>4 / 9=4 / 9>1 / 3>0=0=0=0=0=0\} .
$$

Acknowledgments. We would like to heartily thank A. Avila, A. Eskin, P. Hubert, M. Kontsevich, M. Möller and J.-C. Yoccoz for several discussions related to the material of this paper. Also, we are thankful to the anonymous referees for their comments and suggestions leading to the current version of this article.

The authors thank Collège de France, Institut des Hautes Études Scientifiques (IHES), Hausdorff Research Institute for Mathematics (HIM) and Max Planck Institute for Mathematics (MPIM) for their hospitality during the preparation of this paper.

The first author was supported by the National Science Foundation grant DMS 0800673.

The second author was partially supported by the Balzan Research Project of J. Palis and the French ANR grant "GeoDyM" (ANR-11-BS01-0004).

\section{REFERENCES}

[AEZ] J. Athreya, A. Eskin, A. Zorich, Right-angled billiards and volumes of the moduli spaces of quadratic differentials on $\mathbb{C P}^{1}$, in preparation.

[Au] D. Aulicino, Teichmüller Discs with Completely Degenerate Kontsevich-Zorich Spectrum, arXiv:1205.2359 (2012), 1-74.

[AV] A. Avila and M. Viana, Simplicity of Lyapunov Spectra: Proof of the KontsevichZorich conjecture, Acta Math., 198, (2007), 1-56.

[Ba] M. Bainbridge, Euler characteristics of Teichmüller curves in genus two, Geometry and Topology, 11, (2007), 1887-2073.

[BDV] C. Bonatti, L. Diaz and M. Viana, Dynamics Beyond Uniform Hyperbolicity, Encycl. of Math. Sciences 102, Springer-Verlag, New York, 2005.

[B] I. Bouw, The p-rank of ramified covers of curves, Compositio Math., 126, (2001), 295-322.

[BMo] I. Bouw and M. Möller, Teichmüller curves, triangle groups and Lyapunov exponents, Ann. of Math., 172, (2010), 139-185.

[DHL] V. Delecroix, P. Hubert, and S. Lelièvre, Diffusion for the periodic wind-tree model, arXiv:1107.1810 (2011), 1-28.

[EKZ1] A. Eskin, M. Kontsevich, A. Zorich, Sum of Lyapunov exponents of the Hodge bundle with respect to the Teichmüller geodesic flow, arXiv:1112.5872 (2011), 1106.

[EKZ2] A. Eskin, M. Kontsevich, A. Zorich, Lyapunov spectrum of square-tiled cyclic covers, Journal of Modern Dynamics, 5, no. 2, (2011), 319-353.

[F1] G. Forni, Solutions of the cohomological equation for area-preserving flows on compact surfaces of higher genus, Ann. of Math., 146, no. 2, (1997), 295-344. 
[F2] G. Forni, Deviation of ergodic averages for area-preserving flows on surfaces of higher genus, Annals of Math., 155, no. 1, (2002), 1-103.

[F3] G. Forni, On the Lyapunov exponents of the Kontsevich-Zorich cocycle, Handbook of Dynamical Systems v. 1B, B. Hasselblatt and A. Katok, eds., Elsevier, (2006), 549-580.

[F4] G. Forni, A geometric criterion for the non-uniform hyperbolicity of the KontsevichZorich cocycle (with an appendix by C. Matheus), Journal of Modern Dynamics, 5, no. 2, (2011), 355-395.

[FMt] G. Forni, C. Matheus, An example of a Teichmüller disk in genus 4 with degenerate Kontsevich-Zorich spectrum, arXiv:0810.0023 (2008), 1-8.

[FMZ] G. Forni, C. Matheus and A. Zorich, Square-tiled cyclic covers, Journal of Modern Dynamics, 5, no. 2, (2011), 285-318.

[FMZ2] G. Forni, C. Matheus and A. Zorich, Zero Lyapunov exponents of the Hodge bundle, arXiv:1201.6075 (2012), 1-39 (to appear in Comment. Math. Helv.)

[GH] Ph. Griffiths, J. Harris, Principles of algebraic geometry. Wiley, New York, 1978.

[HK] B. Hasselblatt and A. Katok, Introduction to the Modern Theory of Dynamical Systems, Encycl. Math. and Its Appl. 54 Cambridge University Press, 1995.

[HS] F. Herrlich and G. Schmithüsen, An extraordinary origami curve, Math. Nachr. 281:2, (2008), 219-237.

[IT] Y. Imayoshi and M. Taniguchi, An Introduction to Teichmüller Spaces, Springer Verlag, Tokyo, 1992.

[KM] A. Kappes, M. Möller, Lyapunov spectrum of ball quotients with applications to commensurability questions, arXiv:1207.5433 (2012), 1-37.

[K] M. Kontsevich, Lyapunov exponents and Hodge theory, in "The mathematical beauty of physics", Saclay, 1996. Adv. Ser. Math. Phys. 24. World Scientific, River Edge, NJ, (1997), 318-332.

[M] R. Mañé, Ergodic Theory and Differentiable Dynamics, Springer-Verlag, 1987.

[MT] H. Masur, S. Tabachnikov, Rational billiards and flat structures. Handbook of dynamical systems, Vol. 1A, 1015-1089, North-Holland, Amsterdam, 2002.

[Mc] C. McMullen, Braid groups and Hodge theory, preprint, (2009), 66 pp. (to appear on Math. Ann.)

[Mo] M. Möller, Shimura and Teichmüller curves, Journal of Modern Dynamics, 5, no. 1, (2011), 1-32.

[Na] S. Nag, The Complex Analytic Theory of Teichmüller Spaces, John Wiley \& Sons, Inc., New York, 1988.

[Ro] V. Rokhlin, On the fundamental ideas of measure theory, Mat. Sbornik N.S. 25(67), 1949, 107-150.

[V] W. Veech, Teichmüller Geodesic Flow, Annals of Mathematics, 124, (1986), 441530 .

[Z0] A. Zorich, Asymptotic flag of an orientable measured foliation on a surface. Geometric study of foliations (Tokyo, 1993), 479-498, World Sci. Publ., River Edge, NJ, 1994.

[Z1] A. Zorich, How do the leaves of a closed 1-form wind around a surface, In the collection: "Pseudoperiodic Topology", AMS Translations, Ser. 2, 197, AMS, Providence, RI, (1999), 135-178.

[Z2] A. Zorich, Flat surfaces, in collection "Frontiers in Number Theory, Physics and Geometry. Vol. 1: On random matrices, zeta functions and dynamical systems"; Ecole 
de physique des Houches, France, March 9-21 2003, P. Cartier; B. Julia; P. Moussa; P. Vanhove (Editors), Springer-Verlag, Berlin, (2006), 439-586.

Giovanni Forni: Department of Mathematics, University of MARYland, COLLEge PARK, MD 20742-4015, USA

E-mail address: gforni@math.umd.edu.

Carlos Matheus: CNRS, LaGa, Institut Galilée, Université Paris 13, 99, aVenue Jean-Baptiste Clément, 93430, Villetaneuse, France

E-mail address: matheus@impa.br, matheus@math.univ-paris13.fr.

Anton Zorich: IRMAR, Université de Rennes 1, CAmpus de Beaulieu, 35042, RENNES, FRANCE

E-mail address: anton.zorich@univ-rennes1.fr. 